\title{
THE STRUCTURE OF THE SPACE OF COADJOINT ORBITS OF AN EXPONENTIAL SOLVABLE LIE GROUP
}

\author{
BRADLEY N. CURREY
}

\begin{abstract}
In this paper we address the problem of describing in explicit algebraic terms the collective structure of the coadjoint orbits of a connected, simply connected exponential solvable Lie group $G$. We construct a partition $\wp$ of the dual $\mathfrak{g}^{*}$ of the Lie algebra $\mathfrak{g}$ of $G$ into finitely many $\operatorname{Ad}^{*}(G)$-invariant algebraic sets with the following properties. For each $\Omega \in \wp$, there is a subset $\Sigma$ of $\Omega$ which is a cross-section for the coadjoint orbits in $\Omega$ and such that the natural mapping $\Omega / \operatorname{Ad}^{*}(G) \rightarrow \Sigma$ is bicontinuous. Each $\Sigma$ is the image of an analytic $\operatorname{Ad}^{*}(G)$-invariant function $P$ on $\Omega$ and is an algebraic subset of $\mathfrak{g}^{*}$. The partition $\wp$ has a total ordering such that for each $\Omega \in \wp$, $\bigcup\left\{\Omega^{\prime}: \Omega^{\prime} \leq \Omega\right\}$ is Zariski open. For each $\Omega$ there is a cone $W \subset \mathfrak{g}^{*}$, such that $\Omega$ is naturally a fiber bundle over $\Sigma$ with fiber $W$ and projection $P$. There is a covering of $\Sigma$ by finitely many Zariski open subsets $O$ such that in each $O$, there is an explicit local trivialization $\Theta: P^{-1}(O) \rightarrow W \times O$. Finally, we show that if $\Omega$ is the minimal element of $\wp$ (containing the generic orbits), then its cross-section $\Sigma$ is a differentiable submanifold of $\mathfrak{g}^{*}$. It follows that there is a dense open subset $U$ of $G^{\wedge}$ such that $U$ has the structure of a differentiable manifold and $G^{\wedge} \sim U$ has Plancherel measure zero.
\end{abstract}

\section{INTRODUCTION}

Let $G$ be a connected, simply connected exponential solvable Lie group with Lie algebra $\mathfrak{g}$. Denote by $K$ the canonical bijection from the set $\mathfrak{g}^{*} / G$ of orbits in $\mathfrak{g}^{*}$ under the coadjoint representation $\mathrm{Ad}^{*}$ of $G$ onto the dual $G^{\wedge}$ of $G$. In the nilpotent case, L. Pukanszky in [18] (cf. also [16]) gives a detailed and explicit description of the collective structure on the "generic" coadjoint orbits. He then obtains a very explicit form of the Plancherel theorem for nilpotent groups [16]. Pukanszky's methods actually apply to the "singular" orbits as well, and the resulting description of $\mathfrak{g}^{*} / G$ has found more recent applications. For example in [14], N. V. Pedersen obtains, for any irreducible representation $\pi$ of a nilpotent Lie group, a finite set of explicitly constructible generators for the kernel of $d \pi$ in the enveloping algebra $U\left(\mathfrak{g}_{c}\right)$. L. Corwin and F. Greenleaf in [2] use Pukanszky's description to obtain the spectral decomposition for induced representations for nilpotent groups in terms of the orbit picture. Again the results here are quite explicit; in particular, given the spectral decomposition for an induced representation, a criterion is given for (generic) finite multiplicity.

Received by the editors August 31, 1989 and, in revised form, March 5, 1990 and April 9, 1990. The contents of this paper were presented on January 18,1990 at the 96th Annual Meeting of the American Mathematical Society.

1980 Mathematics Subject Classification (1985 Revision). Primary 22E27; Secondary 22E45. 
In this paper we shall give a detailed and explicit description of the collective structure of the space $\mathfrak{g}^{*} / G$ in the case that $G$ is exponential.

We note that most (but not all) of the above results on spectral decomposition were later obtained by R. Lipsman [10] by somewhat simpler methods where the use of the Pukanszky description is kept to a minimum. Lipsman's approach to spectral decomposition applies to a broad class of groups including the completely solvable groups $[9,11]$. In the exponential case, there is a Plancherel theorem (cf. [5]) and the spectral decomposition of induced and restricted representations has (much more recently) been described by $\mathrm{H}$. Fujiwara $[6,8]$, again by means of the orbit method. However, a criterion for generic finite multiplicity in the exponential case is not known.

We recall the results on the structure of $\mathfrak{g}^{*} / G$ in the nilpotent case. In [18] It is shown that there is an $\operatorname{Ad}^{*}(G)$-invariant Zariski open subset $\Omega$ of $\mathfrak{g}^{*}$, and a subspace $V$ in $\mathfrak{g}^{*}$, such that the coadjoint orbits in $\Omega$ all have the same dimension, and such that the algebraic set $\Sigma=\Omega \cap V$ is a crosssection for $\mathfrak{g}^{*} / G$, that is, $\Sigma$ meets each orbit in $\Omega$ exactly once. There is a rational $\operatorname{Ad}^{*}(G)$-invariant function $P: \Omega \rightarrow \Omega$ such that $\Sigma=P(\Omega)$, and the natural bijection between $\Omega / G$ and $\Sigma$ is a homeomorphism. Finally, there is a subspace $W$ in $\mathfrak{g}^{*}$ and a computable, rational, nonsingular map $\Theta: \Sigma \times W \rightarrow \Omega$ such that for each $\mathfrak{l} \in \Sigma, \Theta(\mathfrak{l}, \cdot)$ is a polynomial map whose graph is the orbit of $\mathfrak{l}$. Recall also that the bijection $K$ is a homeomorphism [1] (where $G^{-}$ carries the hull kernel topology, and $\mathfrak{g}^{*} / G$ the quotient topology). Thus if $G$ is nilpotent, there is a dense, open subset of $G^{\wedge}$ which has the structure of a differentiable manifold.

It is easily seen that Pukanszky's technique yields a partition of $\mathfrak{g}^{*}$ by a finite collection of algebraic subsets $\left\{\Omega_{e}\right\}$ such that for each $\Omega_{e}$, one obtains objects $V_{e}, \Sigma_{e}, W_{e}$, and $\Theta_{e}$ analogous to the above. There is a natural total ordering of $\left\{\Omega_{e}\right\}$ such that the minimal element is $\Omega$, and for each $\Omega_{e}, \bigcup\left\{\Omega_{e^{\prime}}\right.$ : $\left.\Omega_{e^{\prime}} \leq \Omega_{e}\right\}$ is Zariski open. The $\Omega_{e}$ and all associated objects depend only on the choice of a Jordan-Hölder basis for $\mathfrak{g}$; once such a basis is chosen, the constructions for all these objects are quite explicit. The ordering of the $\Omega_{e}$ and the algebraic computability of the cross-sections make this version of Pukanszky's result particularly useful. The partition $\left\{\Omega_{e}\right\}$ has been useful in the solvable case as well. For example in [13], N. V. Pedersen obtains a character formula for an irreducible representation the construction of which depends only on the layer $\Omega_{e}$ containing the associated orbit (cf. also [15]). In the solvable case however, given a layer $\Omega_{e}$, it is not possible to construct a "nice" cross-section $\Sigma_{e}$ for $\Omega_{e}$ as in the nilpotent case. In particular, if $\Omega$ is the Zariski-open layer of Pukanszky, then $\Omega / G$ may not be a manifold even in the algebraic completely solvable case. In this paper we show that in the exponential solvable case there is a finite refinement of the partition $\left\{\Omega_{e}\right\}$ such that in each of the refined layers, one can obtain computable objects analogous to $V, \Sigma, W$, and $\boldsymbol{\theta}$. This refined partition also has a nice ordering, and the layers are algebraic subsets of $\mathfrak{g}^{*}$. Our constructions depend only on a choice of bases for $\mathfrak{g}$ and its complexification $\mathfrak{g}_{c}$ (the latter being a Jordan-Hölder basis). To be more precise, we state an abbreviated version of the main theorem of this paper.

Theorem. Let $G$ be a connected, simply connected exponential solvable Lie group with Lie algebra $\mathfrak{g}$, and let $\left\{Z_{1}, Z_{2}, \ldots, Z_{n}\right\}$ be a Jordan-Hölder basis 
of $\mathfrak{g}_{c}$. Let $f_{j}=Z_{j}^{*} \quad(1 \leq j \leq n)$ be the dual basis in $\mathfrak{g}_{c}^{*}$. Then there is a finite partition $\wp$ of $\mathfrak{g}^{*}$, computable in terms of the above Jordan-Hölder basis, with the following properties:

(a) each $\Omega \in \wp$ is G-invariant,

(b) for a given $\Omega \in \wp$, the dimension of the coadjoint orbits in $\Omega$ is constant,

(c) there is a total ordering $\Omega_{1}<\Omega_{2}<\cdots<\Omega_{u}$ of $\wp$ such that for each $\Omega$, $\bigcup\left\{\Omega^{\prime}: \Omega^{\prime} \leq \Omega\right\}$ is Zariski open in $\mathfrak{g}^{*}$.

Given $\Omega \in \wp$, there is a subspace $V$ in $\mathfrak{g}^{*}$ and a cone $W$ in $\mathfrak{g}^{*}$, and there are associated to $\Omega$ indices $l$ and $\varphi$, for each $j \in l$ a complex valued rational function $p_{j}$ on $\mathfrak{g}^{*}$ and for each $j \in \varphi$, a complex valued rational function $q_{j}$ on $\mathfrak{g}^{*}$ such that

(d) $p_{j}$ is nonsingular on $\Omega$, and $q_{j}$ is nonsingular and nonzero on $\Omega$, and $G$-semiinvariant,

(e) the set $\Sigma=\left\{\mathfrak{l} \in V \cap \Omega: p_{j}(\mathfrak{l})=0, j \in l,\left|q_{j}(\mathfrak{l})\right|^{2}=1, j \in \varphi\right\}$ is a cross-section for the coadjoint orbits in $\Omega$.

Moreover there is an analytic $G$-invariant function $P: \Omega \rightarrow \Omega$ such that $P(\Omega)=\Sigma$, there is a cone $W(\Omega)$ in $\mathfrak{g}^{*}$ and a cover of $\Sigma$ by Zariski open sets $O$ with the following property. For each $O$, there is an analytic map $\Theta: P^{-1}(O) \rightarrow$ $W(\Omega) \times O$ such that for each $\lambda \in O, \Theta^{-1}(\cdot, \lambda)$ is an analytic diffeomorphism of $W(\Omega)$ with the coadjoint orbit of $\lambda$. In particular $\Omega$ is a fiber bundle over $\Sigma$ with fiber $W(\Omega)$ and projection $P$.

The notation and wording of this theorem follows to some extent that of Theorem 1 of [2], in which Pukanszky's result for the nilpotent case is stated in its more general form. We remark that if $G$ is completely solvable, then the bundle structure of each $\Omega$ is trivial. The details of the completely solvable case are in [4]. In any case, if $\Omega$ is the minimal element of $\wp$ and $\Sigma$ its cross-section, then we show that $\Sigma$ is a smooth submanifold of $\mathfrak{g}^{*}$.

In the exponential case, it is known that $K$ is bicontinuous on a dense open subset of $G^{\wedge}$ [7], in fact, there is a computable partition of $\mathfrak{g}^{*} / G$ (which includes a dense open set) on each element of which $K$ is bicontinuous [3]. Combining the above results with Corollary 4.8 of [3], we have the following result.

Theorem. Let $G$ be as in the above theorem. There is a dense open subset $U$ of $G^{\wedge}$ such that $U$ has the structure of a differentiable manifold (whose underlying topology is the relative hull-kernel topology on $U$ ) and $G^{\wedge} \sim U$ has Plancherel measure zero.

\section{A LAYERING OF $\mathfrak{g}^{*}$}

Let $G$ be a connected, simply connected exponential solvable Lie group with Lie algebra $\mathfrak{g}$. We begin by choosing a basis $X_{1}, X_{2}, \ldots, X_{n}$ of $\mathfrak{g}$ with the following properties. Set $\mathfrak{g}_{j}=\operatorname{span}\left\{X_{1}, X_{2}, \ldots, X_{j}\right\}$; we choose our basis so that for some $p \quad(1 \leq p \leq n) \quad \mathfrak{g}_{p}$ is the nilradical of $\mathfrak{g}$, and so that if $\mathfrak{g}_{j}$ is not an ideal, then $\mathfrak{g}_{j+1}$ is an ideal, $1 \leq j<n$. We shall call such a basis a "good basis" of $\mathfrak{g}$. Let $I=\left\{j: \mathfrak{g}_{j}\right.$ is an ideal $\}, I^{\prime}=\{j \in I: j-1 \in I\}$, $I^{\prime \prime}=I \sim I^{\prime}$. Let $e_{j}=X_{j}^{*} \quad(1 \leq j \leq n)$ be the dual basis in $\mathfrak{g}^{*}$. For $\mathfrak{l} \in \mathfrak{g}^{*}$, $X \in \mathfrak{g}$, let $[X, \mathfrak{l}]=\operatorname{ad}^{*} X(\mathfrak{l})$; we denote the coadjoint action of $G$ on $\mathfrak{g}^{*}$ multiplicatively. Consider next the complexification $\mathfrak{g}_{c}$ of $\mathfrak{g}$; we have $\mathfrak{g} \subset \mathfrak{g}_{c}$ 
as a subalgebra, and we extend elements $\mathfrak{l} \in \mathfrak{g}^{*}$ to $\mathfrak{g}_{c}$ in the natural way so that $\mathfrak{g}^{*}=\left\{\mathfrak{l} \in \mathfrak{g}_{c}^{*}: \mathfrak{l}(\bar{Z})=\mathfrak{l}(Z)^{-}\right\}$. Define elements $Z_{j} \quad(1 \leq j \leq n)$ of $\mathfrak{g}_{c}$ as follows. Fix $j, 1 \leq j \leq n$; if $j \in I^{\prime}$ set $Z_{j}=X_{j}$, and if $j \in I^{\prime \prime}$, set $Z_{j-1}=X_{j-1}+i X_{j}$ and $Z_{j}=X_{j-1}-i X_{j}$. The set $\left\{Z_{1}, Z_{2}, \ldots, Z_{n}\right\}$ constitutes a Jordan-Hölder basis of $\mathfrak{g}_{c}$; we shall refer to it as the Jordan-Hölder basis corresponding to the good basis $\left\{X_{1}, X_{2}, \ldots, X_{n}\right\}$ of $\mathfrak{g}$. Let $\mathfrak{s}_{j}$ be the ideal in $\mathfrak{g}_{c}$ spanned by $\left\{Z_{1}, Z_{2}, \ldots, Z_{j}\right\} \quad 1 \leq j \leq n$. Let $f_{j}=Z_{j}^{*} \quad(1 \leq j \leq n)$ be the dual basis in $\mathfrak{g}_{c}^{*}$. Note that for each $j$, if $j \in I^{\prime}$, then $f_{j}=e_{j}$, while if $j \in I^{\prime \prime}$, then

$$
f_{j-1}=\left(e_{j-1}-i e_{j}\right) / 2, \quad f_{j}=\left(e_{j-1}+i e_{j}\right) / 2 .
$$

Let $V_{j}=\operatorname{span}_{\mathbf{R}}\left\{e_{j+1}, \ldots, e_{n}\right\}, W_{j}=\operatorname{span}_{\mathbf{R}}\left\{e_{1}, \ldots, e_{j}\right\} \cong g^{*} / V_{j}, 1 \leq j \leq n$. Let $\pi_{j}: \mathfrak{g}^{*} \rightarrow W_{j}$ the projection parallel to $V_{j}$. Then for each $j, V_{j}$ is a $G$ submodule of $\mathfrak{g}^{*}$, and $W_{j}$ is naturally a $G$-module such that $s \pi_{j}(\mathfrak{l})=\pi_{j}(s \mathfrak{l})$, $s \in G, \mathfrak{l} \in \mathfrak{g}^{*}$. For each $j, 1 \leq j \leq n$, let $j^{\prime}=\max \{i \in I: i<j\}$ and let $j^{\prime \prime}=\min \{i \in I: i \geq j\}$, and let $\gamma_{j} \in \mathfrak{g}^{*}$ be defined by $\left[X, f_{j}\right]=$ $\gamma_{j}(X) f_{j} \bmod \left(\left(V_{j^{\prime \prime}}\right)_{c}\right), X \in \mathfrak{g} . \quad$ Set $C_{j}=\operatorname{ker} \gamma_{j}, 1 \leq j \leq n$. Fix $j \in I$ and let $U$ be the irreducible $G$-submodule of $W_{j}$ canonically isomorphic to $V_{j^{\prime}} / V_{j}$. For each $\mathfrak{l} \in \mathfrak{g}^{*}$ consider the equivalence relation on the affine set $\pi_{j}(\mathfrak{l})+U \subset W_{j}$ induced by the quotient space $W_{j} / G$. It is known that there are essentially four "types" of such equivalence relations: $(0) \pi_{j}(\mathfrak{l})+U$ meets each coadjoint orbit at at most one point, (1) $\pi_{j}(\mathfrak{l})+U$ is contained in a single coadjoint orbit, (2) $\pi_{j}(\mathfrak{l})+U$ meets a one parameter family of orbits, each in a one dimensional affine subset, or (3) generically, $\pi_{j}(\mathfrak{l})+U$ meets a family of orbits parameterized by $S^{0}$ (here $S^{0}=\{-1,1\}$ ) if $\operatorname{dim}(U)=1$, or $S^{1}$ if $\operatorname{dim}(U)=2$. The types (2) and (3) will occur only if $G$ is not nilpotent. In the type (3) case if $\operatorname{dim}(U)=1$, then (generically) the equivalence classes will be two open half lines, while if $\operatorname{dim}(U)=2$, the equivalence classes are "exponential spirals" in $U$. For any $s \in G, \pi_{j}(s \mathfrak{l})+U$ has the same equivalence relation type as $\pi_{j}(\mathfrak{l})+U$. We wish to define $G$-invariant layers in which, for each $j$, the equivalence relation type of $\pi_{j}(\mathfrak{l})+U$ is "constant."

For each $\mathfrak{l} \in \mathfrak{g}^{*}$, and for any subset $\mathfrak{h}$ of $\mathfrak{g}_{c}$, let $\mathfrak{h}^{\mathfrak{l}}=\left\{Y \in \mathfrak{g}_{c}: \mathfrak{l}([Y, X])=0\right.$, for all $X \in \mathfrak{h}\}$, and set $R(\mathfrak{l}, \mathfrak{h})=\mathfrak{h}^{\mathfrak{l}} \cap \mathfrak{h}$. Let $L_{j}(\mathfrak{l})=\mathfrak{s}_{j}^{\mathfrak{l}}, \quad 1 \leq j \leq n$, and let $R(\mathfrak{l})=L_{n}(\mathfrak{l})$. For each $\mathfrak{l}$, we define a subset of indices $J_{\mathfrak{l}} \subset\{1,2, \ldots, n\}$ by $J_{\mathfrak{l}}=\left\{j: L_{j}(\mathfrak{l}) \neq L_{j-1}(\mathfrak{l})\right\}$. It is easily seen that $J_{\mathfrak{l}}=\left\{j: \mathfrak{s}_{j}+R(\mathfrak{l}) \neq \mathfrak{s}_{j-1}+R(\mathfrak{l})\right\}$. Let $E=\left\{J_{\mathfrak{l}}: \mathfrak{l} \in \mathfrak{g}^{*}\right\}$ and for each $J \in E$, let $\Omega_{J}=\left\{\mathfrak{l} \in \mathfrak{g}^{*}: J_{\mathfrak{l}}=J\right\}$. Each $\Omega_{J}$ is a $G$-invariant algebraic set, and the set $E$ has a total ordering such that for each $J \in E, \bigcup\left\{\Omega_{J^{\prime}}: J^{\prime} \leq J\right\}$ is a Zariski-open subset of $\mathfrak{g}^{*}$. As is shown in [13], there are semi-invariant polynomials $\left\{Q_{J}: J \in E\right\}$ such that

$$
\Omega_{J}=\left\{\mathfrak{l} \in g^{*}: Q_{J^{\prime}}(\mathfrak{l})=0, J^{\prime}<J, Q_{J}(\mathfrak{l}) \neq 0\right\} .
$$

We now define for each $j(1 \leq j \leq n)$ and $\mathfrak{l} \in \mathfrak{g}^{*}$, the "type" $\tau_{j}(\mathfrak{l})$ of $\mathfrak{l}$ at $j$ as follows:

$$
\begin{aligned}
& \tau_{j}(\mathfrak{l})=0, \text { if for every } \mathfrak{l}^{\prime} \in \pi_{j^{\prime}}^{-1}(\mathfrak{l}), L_{j^{\prime}}\left(\mathfrak{l}^{\prime}\right)=L_{j^{\prime \prime}}\left(\mathfrak{l}^{\prime}\right), \\
& \tau_{j}(\mathfrak{l})=1, \text { if } \operatorname{dim}\left(L_{j^{\prime}}(\mathfrak{l}) \cap C_{j} / L_{j^{\prime \prime}}(\mathfrak{l}) \cap C_{j}\right)=j^{\prime \prime}-j^{\prime}, \\
& \tau_{j}(\mathfrak{l})=2, \text { if } j^{\prime \prime}-j^{\prime}=2 \text { and } \operatorname{dim}\left(L_{j^{\prime}}(\mathfrak{l}) \cap C_{j} / L_{j^{\prime \prime}}(\mathfrak{l}) \cap C_{j}\right)=1 \text {, and } \\
& \tau_{j}(\mathfrak{l})=3 \text {, if there is } \mathfrak{l}^{\prime} \in \pi_{j^{\prime}}^{-1}(\mathfrak{l}) \text { such that } L_{j^{\prime}}\left(\mathfrak{l}^{\prime}\right) \neq L_{j^{\prime \prime}}\left(\mathfrak{l}^{\prime}\right) \text { but }
\end{aligned}
$$

$$
L_{j^{\prime}}\left(\mathfrak{l}^{\prime}\right) \cap C_{j}=L_{j^{\prime \prime}}\left(\mathfrak{l}^{\prime}\right) \cap C_{j}
$$


It can be seen from the proof of Proposition 5 in [17] that if

$$
\operatorname{dim}\left(L_{j^{\prime}}(\mathfrak{l}) \cap C_{j} / L_{j^{\prime \prime}}(\mathfrak{l}) \cap C_{j}\right)=1,
$$

then $\operatorname{dim}\left(L_{j^{\prime}}(\mathfrak{l}) / L_{j^{\prime \prime}}(\mathfrak{l})\right)=1$ (hence $\tau_{j}$ is defined for all $\mathfrak{l}$ ), and that $\tau_{j}(\mathfrak{l})=0$ (resp. 1, 2, 3) if and only if the equivalence relation on $\pi_{j}(\mathfrak{l})+U$ is of type (0) (resp. (1), (2), (3)) (these facts will also follow from the sequel). Simple examples show that the functions $\tau_{j}$ are not necessarily constant on the layers $\Omega_{J}$; note however that for any $\mathfrak{l} \in \Omega_{J}, \tau_{j}(\mathfrak{l})=0 \operatorname{implies} \operatorname{dim}\left(\pi_{j^{\prime \prime}}(G \mathfrak{l})\right)$ $=\operatorname{dim}\left(\pi_{j^{\prime}}(G \mathfrak{l})\right)$ (hence $\left.j \notin J\right)$, while $\tau_{j}(\mathfrak{l})=1$ implies $\operatorname{dim}\left(\pi_{j^{\prime \prime}}(G \mathfrak{l})\right)=$ $\operatorname{dim}\left(\pi_{j^{\prime}}(G \mathfrak{l})\right)+j-j^{\prime}$ (hence $\left.j \in J\right)$. In order to obtain the desired layering, we shall first define a partition of each $\Omega_{J}$ into $G$-invariant algebraic sets in which the conditions $\tau_{j}(\mathfrak{l})=$ constant are determined by explicit polynomials.

Let $J \in \mathfrak{j}, J \neq \varnothing$, and write $J=\left\{j_{1}<j_{2}<\cdots<j_{d}\right\}$. Let $\mathfrak{l} \in \Omega_{J}$. Denote by $\mathfrak{p}(\mathfrak{l})$ the complex subalgebra $\mathfrak{p}(\mathfrak{l})=\sum_{j} R\left(\mathfrak{l}, \mathfrak{s}_{j}\right) ; \mathfrak{p}(\mathfrak{l})$ is totally isotropic with respect to the alternating bilinear form $B_{\mathfrak{l}}$ on $\mathfrak{g}_{c}$ associated with $\mathfrak{l}$. Let $j(\mathfrak{l})=\left\{j: \mathfrak{s}_{j}+\mathfrak{p}(\mathfrak{l}) \neq \mathfrak{s}_{j-1}+\mathfrak{p}(\mathfrak{l})\right\}$. Since $\mathfrak{p}(\mathfrak{l}) \supset R(\mathfrak{l})$, we have $J_{\mathfrak{l}} \supset j(\mathfrak{l})$, and $\operatorname{card}(j(\mathfrak{l}))=\operatorname{codim}(\mathfrak{p}(\mathfrak{l}))=d / 2$. Let $i(\mathfrak{l})=J_{\mathfrak{l}} \sim j(\mathfrak{l})$. We define a sequence of subalgebras $\mathfrak{g}_{c}=\mathfrak{h}_{0}(\mathfrak{l}) \supset \mathfrak{h}_{1}(\mathfrak{l}) \supset \cdots \supset \mathfrak{h}_{d / 2}(\mathfrak{l})$ as follows. Set $\mathfrak{h}_{0}(\mathfrak{l})=\mathfrak{g}_{c}$ and set $i_{1}=j_{1}$. Assume that for some $k, 0 \leq k<d / 2, \mathfrak{h}_{k}(\mathfrak{l})$ is defined and $\operatorname{dim}_{\mathbf{C}}\left(\mathfrak{g}_{c} / \mathfrak{h}_{k}(\mathfrak{l})\right)=k$; from dimensionality considerations we have $\mathfrak{h}_{k}(\mathfrak{l}) \neq$ $R\left(\mathfrak{l}, \mathfrak{h}_{k}(\mathfrak{l})\right)$. Let $i_{k+1}$ be the smallest index $i$ such that $\mathfrak{s}_{i} \cap \mathfrak{h}_{k}(\mathfrak{l}) \not \subset R\left(\mathfrak{l}, \mathfrak{h}_{k}(\mathfrak{l})\right)$ and set

$$
\mathfrak{h}_{k+1}(\mathfrak{l})=\left(\mathfrak{s}_{i_{k+1}} \cap \mathfrak{h}_{k}(\mathfrak{l})\right)^{\mathfrak{l}} \cap \mathfrak{h}_{k}(\mathfrak{l})
$$

Then $\mathfrak{h}_{k+1}(\mathfrak{l})$ is a complex codimension one subalgebra of $\mathfrak{h}_{k}(\mathfrak{l})$. We terminate the process when $k+1=d / 2$, thus obtaining indices $i_{1}, i_{2}, \ldots, i_{d / 2}$. Note that by construction, if $\mathfrak{h}_{k}(\mathfrak{l}) \supset R(\mathfrak{l})$ then $\mathfrak{h}_{k+1}(\mathfrak{l}) \supset R(\mathfrak{l}), 0 \leq k<d$. It follows immediately that

$$
\mathfrak{h}_{k}(\mathfrak{l}) \supset R\left(\mathfrak{l}, \mathfrak{h}_{k}(\mathfrak{l})\right) \supset R(\mathfrak{l})
$$

Note also that for each $k \quad(1 \leq k \leq d / 2)$, and $j$ such that $i_{k} \leq j<i_{k+1}$, $L_{j}(\mathfrak{l}) \subset \mathfrak{h}_{k}(\mathfrak{l})$. For each $k=1,2, \ldots, d / 2$, let $j\left(i_{k}\right)$ be the smallest index $j$ such that

$$
\mathfrak{s}_{j} \cap \mathfrak{h}_{k-1}(\mathfrak{l}) \not \subset \mathfrak{h}_{k}(\mathfrak{l})
$$

The following lemma generalizes Lemma 3.2 of [3] and the statements preceding Lemma 1.1 of [4].

Lemma 1.1. For each $k, 1 \leq k \leq d / 2, i_{k} \in J, j\left(i_{k}\right) \in J, i_{k}<j\left(i_{k}\right)$, and for $k<d / 2, i_{k}<i_{k+1}$. Moreover, $\mathfrak{h}_{d / 2}(\mathfrak{l})=\mathfrak{p}(\mathfrak{l}), i(\mathfrak{l})=\left\{i_{1}, i_{2}, \ldots, i_{d / 2}\right\}$, and $j(\mathfrak{l})=\left\{j\left(i_{1}\right), j\left(i_{2}\right), \ldots, j\left(i_{d / 2}\right)\right\}$.

Proof. If $i_{k} \notin J$, then there is $Z \in R(\mathfrak{l})$ such that $\mathfrak{s}_{i_{k}}=\mathbf{C Z}+\mathfrak{s}_{i_{k}-1}$. But since $R(\mathfrak{l}) \subset R\left(\mathfrak{l}, \mathfrak{h}_{k-1}(\mathfrak{l})\right)$,

$$
\mathfrak{s}_{i_{k}} \cap \mathfrak{h}_{k-1}(\mathfrak{l})=\mathbf{C Z}+\left(\mathfrak{s}_{i_{k}-1} \cap \mathfrak{h}_{k-1}(\mathfrak{l})\right) \subset R\left(\mathfrak{l}, \mathfrak{h}_{k-1}(\mathfrak{l})\right)
$$

a contradiction. If $j\left(i_{k}\right) \notin J$, there is $Z \in R(\mathfrak{l})$ such that $\mathfrak{s}_{j\left(i_{k}\right)}=\mathbf{C Z}+\mathfrak{s}_{j\left(i_{k}\right)-1}$. Since $R(\mathfrak{l}) \subset \mathfrak{h}_{k}(\mathfrak{l})$,

$$
\mathfrak{s}_{j\left(i_{k}\right)} \cap \mathfrak{h}_{k-1}(\mathfrak{l})=\mathbf{C Z}+\left(\mathfrak{s}_{j\left(i_{k}\right)-1} \cap \mathfrak{h}_{k-1}(\mathfrak{l})\right) \subset \mathfrak{h}_{k}(\mathfrak{l}),
$$

a contradiction. 
Now by definition of $i_{k}, \mathfrak{s}_{i_{k}-1} \cap \mathfrak{h}_{k-1}(\mathfrak{l}) \subset R\left(\mathfrak{l}, \mathfrak{h}_{k-1}(\mathfrak{l})\right)$; hence

$$
\left[\mathfrak{s}_{i_{k}} \cap \mathfrak{h}_{k-1}(\mathfrak{l}), \mathfrak{s}_{i_{k}} \cap \mathfrak{h}_{k-1}(\mathfrak{l})\right] \subset \operatorname{ker}(\mathfrak{l})
$$

so that $\mathfrak{h}_{i_{k}} \cap \mathfrak{h}_{k-1}(\mathfrak{l}) \subset \mathfrak{h}_{k}(\mathfrak{l})$. Thus $i_{k}<j\left(i_{k}\right)$. Similarly,

$$
\mathfrak{s}_{i_{k}} \cap \mathfrak{h}_{k}(\mathfrak{l})=\mathfrak{s}_{i_{k}} \cap \mathfrak{h}_{k-1}(\mathfrak{l}) \subset R\left(\mathfrak{l}, \mathfrak{h}_{k}(\mathfrak{l})\right),
$$

and so by definition, $i_{k}<i_{k+1}$. This proves the first statement of the lemma.

To show that $\mathfrak{h}_{d / 2}(1)=\mathfrak{p}(\mathfrak{l})$, it is enough to show containment one way. Let $Z \in R\left(\mathfrak{l}, \mathfrak{s}_{t}\right)$ (some $t$ ) and suppose that $Z \in \mathfrak{h}_{k}(\mathfrak{l})$ for some $k, 0 \leq k<d$. We show that $Z \in \mathfrak{h}_{k+1}(\mathfrak{l})$. If $t<i_{k+1}$, then by definition of $i_{k+1}, Z \in \mathfrak{s}_{t} \cap \mathfrak{h}_{k}(\mathfrak{l}) \subset$ $R\left(\mathfrak{l}, \mathfrak{h}_{k}(\mathfrak{l})\right) \subset \mathfrak{h}_{k+1}(\mathfrak{l})$, while if $t \geq i_{k+1}$, then

$$
Z \in R\left(\mathfrak{l}, \mathfrak{s}_{t}\right) \cap \mathfrak{h}_{k}(\mathfrak{l}) \subset\left(\mathfrak{s}_{t} \cap \mathfrak{h}_{k}(\mathfrak{l})\right)^{\mathfrak{l}} \cap \mathfrak{h}_{k}(\mathfrak{l}) \subset \mathfrak{h}_{k+1}(\mathfrak{l})
$$

Since $Z \in \mathfrak{h}_{0}(\mathfrak{l})=\mathfrak{g}_{c}$, it follows that $Z \in \mathfrak{h}_{d / 2}(\mathfrak{l})$.

Next we show that $i(\mathfrak{l})=\left\{i_{1}, i_{2}, \ldots, i_{d / 2}\right\}$, and for this it suffices to show that $i_{k} \notin j(\mathfrak{l}), 1 \leq k \leq d$. But this follows from the fact that for each $k<d$,

$$
R\left(\mathfrak{l}, \mathfrak{h}_{k}(\mathfrak{l})\right) \subset R\left(\mathfrak{l}, \mathfrak{h}_{k+1}(\mathfrak{l})\right) \subset \mathfrak{p}(\mathfrak{l}),
$$

so that

$$
\mathfrak{s}_{i_{k}} \subset \mathfrak{s}_{i_{k}-1}+R\left(\mathfrak{l}, \mathfrak{h}_{k-1}(\mathfrak{l})\right) \subset \mathfrak{s}_{i_{k}-1}+\mathfrak{p}(\mathfrak{l}) .
$$

Finally, to see that $j(\mathfrak{l})=\left\{j\left(i_{1}\right), j\left(i_{2}\right), \ldots, j\left(i_{d / 2}\right)\right\}$, note first that for each $k$, if $j \notin j(\mathfrak{l}), s_{j} \subset s_{j-1}+\mathfrak{h}_{k}(\mathfrak{l})$ and hence $\mathfrak{s}_{j} \cap \mathfrak{h}_{k-1}(\mathfrak{l}) \subset \mathfrak{s}_{j-1} \cap \mathfrak{h}_{k-1}(\mathfrak{l})+\mathfrak{h}_{k}(\mathfrak{l})$. If $j\left(i_{k}\right) \notin j(\mathfrak{l})$, this implies $\mathfrak{s}_{j} \cap \mathfrak{h}_{k-1}(\mathfrak{l}) \subset \mathfrak{h}_{k}(\mathfrak{l})$, a contradiction. On the other hand, if $j \in j(\mathfrak{l})$, let $k_{0}$ be the smallest $k, 1 \leq k \leq d / 2$, such that $\mathfrak{s}_{j} \subset \mathfrak{s}_{j-1}+\mathfrak{h}_{k}(\mathfrak{l})$. We show that $j=j\left(i_{k_{0}}\right)$. Now choice of $k_{0}$ implies that $\mathfrak{s}_{j} \cap \mathfrak{h}_{k_{0}-1}(\mathfrak{l}) \not \subset \mathfrak{h}_{k_{0}}(\mathfrak{l})$, hence $j \geq j\left(i_{k_{0}}\right)$. But $j>j\left(i_{k_{0}}\right)$ implies

$$
\mathfrak{s}_{j} \subset \mathfrak{s}_{j-1}+\mathfrak{h}_{k_{0}-1}(\mathfrak{l}) \subset \mathfrak{s}_{j-1}+\mathfrak{s}_{j\left(i_{k_{0}}\right)}+\mathfrak{h}_{k_{0}}(\mathfrak{l})=\mathfrak{s}_{j-1}+\mathfrak{h}_{k_{0}}(\mathfrak{l}),
$$

contradicting choice of $k_{0}$. This finishes the proof of the lemma. Q.E.D.

Now for each $\mathfrak{l} \in \mathfrak{g}^{*}$, let us denote the pair $(i(\mathfrak{l}), j(\mathfrak{l}))$ by $\alpha(\mathfrak{l})$; it is easily seen that for each $s \in G, \alpha(\mathfrak{l})=\alpha(s \mathfrak{l})$. Let $A_{J}=\left\{\alpha(\mathfrak{l}): \mathfrak{l} \in \Omega_{J}\right\}$ and for each $\alpha \in A_{J}$, let $\Omega_{\alpha}=\left\{\mathfrak{l} \in \Omega_{J}: \alpha(\mathfrak{l})=\alpha\right\}$.

Proposition 1.2. The set $\left\{\alpha: \alpha \in A_{J}, J \in E\right\}$ has a total ordering $\ll$, such that for each $\alpha, \bigcup\left\{\Omega_{\alpha^{\prime}}: \alpha^{\prime} \ll \alpha\right\}$ is a Zariski-open subset of $\mathfrak{g}^{*}$.

Proof. We first let $A_{J}$ have the total ordering < defined by the lexographic ordering on the set of $d / 2$-tuples $\left\{j(\mathfrak{l})=\left(j\left(i_{1}\right), j\left(i_{2}\right), \ldots, j\left(i_{d / 2}\right)\right):(i(\mathfrak{l}), j(\mathfrak{l}))=\right.$ $\left.\alpha \in A_{J}\right\}$. We claim that for each $\alpha \in A_{J}$, there is a complex-valued polynomial $P_{\alpha}$ on $\mathfrak{g}^{*}$, such that

$$
\Omega_{\alpha}=\left\{\mathfrak{l} \in \Omega_{J}: P_{\alpha^{\prime}}(\mathfrak{l})=0, \alpha^{\prime}<\alpha, P_{\alpha}(\mathfrak{l}) \neq 0\right\} ;
$$

in particular each $\Omega_{\alpha}$ is a $G$-invariant algebraic subset of $\Omega_{J}$. This is easily seen as follows. Let $\alpha \in A_{J}, \alpha=\left(i_{1}, i_{2}, \ldots, i_{d / 2}, j\left(i_{1}\right), j\left(i_{2}\right), \ldots, j\left(i_{d / 2}\right)\right)$. Suppose first that $\alpha$ is the minimal element of $A_{J}$. For each $\mathfrak{l} \in \Omega_{J}$ define $Z_{i_{1}}(\mathfrak{l})=Z_{i_{1}}, Z_{j\left(i_{1}\right)}(\mathfrak{l})=Z_{j\left(i_{1}\right)}$. Note that $i_{1}=i_{1}(\mathfrak{l})$ for every $\mathfrak{l} \in \Omega_{J}$, and we have $j\left(i_{1}\right)(\mathfrak{l})=j\left(i_{1}\right)$ if and only if $\mathfrak{l}\left(\left[Z_{j\left(i_{1}\right)}(\mathfrak{l}), Z_{i_{1}}(\mathfrak{l})\right]\right) \neq 0$, in which case $i_{2}(\mathfrak{l})=i_{2}$. For each $\mathfrak{l} \in \Omega_{J}$ set

$$
Z_{i_{2}}(\mathfrak{l})=\mathfrak{l}\left(\left[Z_{j\left(i_{1}\right)}, Z_{i_{1}}(\mathfrak{l})\right]\right) Z_{i_{2}}-\mathfrak{l}\left(\left[Z_{i_{2}}, Z_{i_{1}}(\mathfrak{l})\right]\right) Z_{j\left(i_{1}\right)}
$$


and

$$
Z_{j\left(i_{2}\right)}(\mathfrak{l})=\mathfrak{l}\left(\left[Z_{j\left(i_{1}\right)}, Z_{i_{1}}(\mathfrak{l})\right]\right) Z_{j\left(i_{2}\right)}-\mathfrak{l}\left(\left[Z_{j\left(i_{2}\right)}, Z_{i_{1}}(\mathfrak{l})\right]\right) Z_{j\left(i_{1}\right)}
$$

Then for every $\mathfrak{l} \in \Omega_{J}$ we have $j\left(i_{1}\right)(\mathfrak{l})=j\left(i_{1}\right)$ and $j\left(i_{2}\right)(\mathfrak{l})=j\left(i_{2}\right)$ if and only if $\mathfrak{l}\left(\left[Z_{j\left(i_{1}\right)}(\mathfrak{l}), Z_{i_{1}}(\mathfrak{l})\right]\right) \mathfrak{l}\left(\left[Z_{j\left(i_{2}\right)}(\mathfrak{l}), Z_{i_{2}}(\mathfrak{l})\right]\right) \neq 0$. Continuing in this way we obtain for each $\mathfrak{l} \in \Omega_{J}$ elements $Z_{i_{1}}(\mathfrak{l}), Z_{i_{2}}(\mathfrak{l}), \ldots, Z_{i_{d / 2}}(\mathfrak{l}), Z_{j\left(i_{1}\right)}(\mathfrak{l}), Z_{j\left(i_{2}\right)}(\mathfrak{l}), \ldots$, $Z_{j\left(i_{d / 2}\right)}(\mathfrak{l})$ belonging to $\mathfrak{g}_{c}$ which depend polynomially on $\mathfrak{l}$ and such that $\mathfrak{l} \in \Omega_{\alpha}$ if and only if $P_{\alpha}(\mathfrak{l}) \neq 0$ where

$$
P_{\alpha}(\mathfrak{l})=\mathfrak{l}\left(\left[Z_{j\left(i_{1}\right)}(\mathfrak{l}), Z_{i_{1}}(\mathfrak{l})\right]\right) \mathfrak{l}\left(\left[Z_{j\left(i_{2}\right)}(\mathfrak{l}), Z_{i_{2}}(\mathfrak{l})\right]\right) \cdots \mathfrak{l}\left(\left[Z_{j\left(i_{d / 2}\right)}(\mathfrak{l}), Z_{i_{d / 2}}(\mathfrak{l})\right]\right) .
$$

Now assume that $\alpha$ is not the minimal element of $A_{J}$ and that for each $\alpha^{\prime}<\alpha$, we have defined a polynomial function $P_{\alpha^{\prime}}(\mathfrak{l})$ such that $\Omega_{\alpha^{\prime}}=\left\{\mathfrak{l} \in \Omega_{J}\right.$ : $\left.P_{\alpha^{\prime \prime}}(\mathfrak{l})=0, \alpha^{\prime \prime}<\alpha^{\prime}, P_{\alpha^{\prime}}(\mathfrak{l}) \neq 0\right\}$. Then for $\mathfrak{l} \in \Omega_{J}$, we have $\mathfrak{l} \in \bigcup\left\{\Omega_{\beta}: \beta \geq \alpha\right\}$ if and only if for every $\alpha^{\prime}<\alpha, P_{\alpha^{\prime}}(\mathfrak{l})=0$. Now for $\mathfrak{l} \in \bigcup\left\{\Omega_{\beta}: \beta \geq \alpha\right\}$, we construct $P_{\alpha}(\mathfrak{l})$ in the same way as above, so that for such $\mathfrak{l}$, we have $\mathfrak{l} \in \Omega_{\alpha}$ if and only if $P_{\alpha}(\mathfrak{l}) \neq 0$. This proves the claim.

The set $\left\{\alpha: \alpha \in A_{J}, J \in \mathfrak{j}\right\}$ then has a total ordering $\ll$ defined as follows: if $\alpha \in A_{J}$ and $\alpha^{\prime} \in A_{J^{\prime}}$; then $\alpha \ll \alpha^{\prime}$ when $J<J^{\prime}$, or if $J=J^{\prime}$, when $\alpha<\alpha^{\prime}$. Now for each $\alpha \in A_{J}$,

$$
\left.\bigcup\left\{\Omega_{\alpha^{\prime}}: \alpha^{\prime} \ll \alpha\right\}=\left\{\mathfrak{l}: \sum_{J^{\prime}<J} Q_{J^{\prime}}(\mathfrak{l})+\left[Q_{J}(\mathfrak{l})\left(\sum_{\alpha^{\prime} \ll \alpha} P_{\alpha^{\prime}}(\mathfrak{l})^{2}\right)\right] \neq 0\right\}\right),
$$

and the proof is complete. Q.E.D.

Remark. The above partition of $\Omega_{J}$ into the sets $\left\{\Omega_{\alpha}\right\}$ has been considered in the nilpotent case by L. Pukanszky [18] and N. V. Pedersen [12]. Let $J=\left\{j_{r}: 1 \leq r \leq d\right\}$, and let $M_{J}(\mathfrak{l})$ denote the skew-symmetric matrix $\left[\mathfrak{l}\left(\left[Z_{j_{r}}, Z_{j_{s}}\right]\right)\right]_{1 \leq r, s \leq d}$. It is easily seen that the condition $P_{\alpha}(\mathfrak{l}) \neq 0$ is equivalent to the condition that each of a certain set of minors of $M_{J}(\mathfrak{l})$ does not vanish. It follows that there is a covering $\left\{O_{\alpha}\right\}$ of Zariski-open subsets of $\Omega_{J}$, such that for each $\alpha$,

$$
\Omega_{\alpha}=\Omega_{J} \sim \bigcup\left\{O_{\alpha^{\prime}}: \alpha^{\prime}<\alpha\right\} .
$$

Pukanszky's construction of the cross-section for $\Omega_{J} / G$ requires this covering (cf. [18, Chapter II, p. 515]), though the cross-section is ultimately defined for all of $\Omega_{J} / G$. In the context of solvable groups however, the partition $\left\{\Omega_{\alpha}\right\}$ is necessary since the type functions $\tau_{j}$ may not be constant on the sets $O_{\alpha}$. We shall see that in each $\Omega_{\alpha}$, the condition that $\tau_{j}$ be constant is a polynomial condition.

Fix $J \in E, J \neq \varnothing, \mathfrak{l} \in \Omega_{J}$ and let $d=\operatorname{dim}(G \mathfrak{l})$. For each $j \in J \cap I$, if $j-j^{\prime}=1$ (resp. $j-j^{\prime}=2$ ) choose $X_{j}$ (resp. $\left.X_{j}, X_{j-1}\right)$ a basis of $L_{j^{\prime}}(\mathfrak{l})$ modulo $L_{j}(\mathfrak{l})$. Then the mapping $R^{d} \rightarrow G \mathfrak{l}$ given by

$$
\left(t_{1}, t_{2}, \ldots, t_{d}\right) \rightarrow \exp \left(t_{1} X_{j_{1}}\right) \exp \left(t_{2} X_{j_{2}}\right) \cdots \exp \left(t_{d} X_{j_{d}}\right) \mathfrak{l}
$$

is a diffeomorphism. We shall give a construction which shows that for each set $\Omega_{\alpha}$, there is a covering of $\Omega_{\alpha}$ by Zariski-open subsets $O$ such that the $X_{j}$ can be chosen so as to vary analytically with $\mathfrak{l} \in O$. This construction generalizes that of the proof of Lemma 1.1 of [4]; unfortunately the extra complications encountered in the exponential case are considerable. 
For $\alpha \in A_{J}, \alpha=(i, j)$, and write $i=\left\{i_{1}, i_{2}, \ldots, i_{d / 2}\right\}, j=\left\{j\left(i_{1}\right), j\left(i_{2}\right)\right.$, $\left.\ldots, j\left(i_{d / 2}\right)\right\}$. Let $K_{0}=\left\{k: i_{k} \in I^{\prime}\right\}, K_{1}=\left\{k: i_{k} \notin I, i_{k}+1 \notin J\right\}, K_{2}=$ $\left\{k: i_{k} \in I^{\prime \prime}, i_{k}-1=j\left(i_{r}\right)\right.$ for some $\left.r<k\right\}, K_{3}=\left\{k: i_{k} \notin I, i_{k}+1=j\left(i_{r}\right)\right.$ for some $r \leq k\}, K_{4}=\left\{k: i_{k} \notin I, i_{k}+1=i_{k+1}\right\}$, and $K_{5}=\left\{k: i_{k} \in\right.$ $\left.I^{\prime \prime}, i_{k}-1=i_{k-1}\right\}=K_{4}+1$. From the properties of the sequences $i$ and $j$ proved in Lemma 1.1 , it follows that $\{1,2, \ldots, d / 2\}=K_{0} \cup K_{1} \cup \cdots \cup K_{5}$ (as a disjoint union).

Lemma 1.3. There is a covering $F$ of $\Omega_{\alpha}$ by finitely many Zariski open subsets such that for each $O \in F$, and for each $k, 1 \leq k \leq d / 2$, there are analytic functions $X_{k}$ and $Y_{k}$ from $O$ into $\mathfrak{g}$ and an analytic function $\phi_{k}$ from $O$ into $S^{1}$ having the following properties.

(i) The maps $\mathfrak{l} \rightarrow \phi_{k}(\mathfrak{l}) X_{k}(\mathfrak{l}), \mathfrak{l} \rightarrow \phi_{k}(\mathfrak{l}) Y_{k}(\mathfrak{l})$ extend to rational functions from $\Omega_{\alpha}$ into $\mathfrak{g}_{c}$ and are independent of the open set $O \in F$.

(ii) For each $\mathfrak{l} \in O, X_{k}(\mathfrak{l}) \in \mathfrak{s}_{j\left(i_{k}\right)^{\prime \prime}} \sim \mathfrak{s}_{j\left(i_{k}\right)^{\prime}}$, and $Y_{k}(\mathfrak{l}) \in \mathfrak{s}_{i_{k}^{\prime \prime}} \sim \mathfrak{s}_{i_{k}^{\prime}}$.

(iii) For each $\mathfrak{l} \in O, \mathfrak{l}\left(\left[X_{k}(\mathfrak{l}), Y_{k}(\mathfrak{l})\right]\right) \neq 0$, and for each $r \quad(1 \leq r \leq k-1)$, we have $\left.\left.\left.\mathfrak{l}\left(\left[X_{k}(\mathfrak{l}), X_{r}(\mathfrak{l})\right]\right)=\mathfrak{l}\left(Y_{k}(\mathfrak{l}), X_{r}(\mathfrak{l})\right]\right)=\mathfrak{l}\left(Y_{k}(\mathfrak{l}), Y_{r}(\mathfrak{l})\right]\right)=\mathfrak{l}\left(Y_{k}(\mathfrak{l}), \bar{Y}_{r}(\mathfrak{l})\right]\right)=0$.

(iv) If $k \in K_{0} \cup K_{1} \cup K_{2} \cup K_{3}$, then for each $\mathfrak{l} \in O, X_{k}(\mathfrak{l}) \in \mathfrak{h}_{k-1}(\mathfrak{l})$, and $Y_{k}(\mathfrak{l}) \in \mathfrak{h}_{k-1}(\mathfrak{l})$. Moreover if $k \in K_{0} \cup K_{1} \cup K_{2}, \mathfrak{h}_{k}(\mathfrak{l})=\mathfrak{h}_{k-1}(\mathfrak{l}) \cap\left\{Y_{k}(\mathfrak{l})\right\}^{\mathfrak{l}}$.

(v) If $k \in K_{4}$, then for each $\mathfrak{l} \in O$, each of $X_{k}(\mathfrak{l}), X_{k+1}(\mathfrak{l}), Y_{k}(\mathfrak{l})$, and $Y_{k+1}(\mathfrak{l})$ belong to $\mathfrak{h}_{k-1}(\mathfrak{l})$. Moreover $\mathfrak{h}_{k+1}(\mathfrak{l})=\mathfrak{h}_{k-1}(\mathfrak{l}) \cap\left\{Y_{k}(\mathfrak{l}), Y_{k+1}(\mathfrak{l})\right\}^{\mathfrak{l}}$.

Suppose that for some $k \quad(1 \leq k \leq d / 2), j\left(i_{k}\right) \notin I^{\prime}$ and $\bar{Z}_{j\left(i_{k}\right)} \neq Z_{j\left(i_{r}\right)}$, for $r<k$. Suppose further that $k \notin K_{3}$. Then there is an analytic function $\mathfrak{l} \rightarrow X_{k}(\mathfrak{l})^{\sim} \in \mathfrak{g}$ satisfying the following.

(i) The map $\mathfrak{l} \rightarrow \phi_{k}(\mathfrak{l}) X_{k}(\mathfrak{l})^{\sim}$ extends to a rational function on $\Omega_{\alpha}$ into $\mathfrak{g}_{c}$ which is independent of the open set $O$.

(ii) For each $\mathfrak{l} \in O, X_{k}(\mathfrak{l})^{\sim} \in \mathfrak{s}_{j\left(i_{k}\right)^{\prime \prime}}$, the elements $X_{k}(\mathfrak{l})$ and $X_{k}(\mathfrak{l})^{\sim}$ are linearly independent modulo $\mathfrak{s}_{j\left(i_{k}\right)^{\prime}}$, and $\mathfrak{l}\left(\left[X_{k}(\mathfrak{l})^{\sim}, Y_{k}(\mathfrak{l})\right]\right)=0$.

(iii) In this case $j\left(i_{k}\right) \notin I$ unless $k \in K_{4}$ and $j\left(i_{k}\right)-1=j\left(i_{k+1}\right)$, and if $k \notin K_{\mathbf{4}}$, then for each $\mathfrak{l} \in \Omega_{\alpha}, X_{k}(\mathfrak{l})^{\sim} \in \mathfrak{h}_{k}(\mathfrak{l})$.

Proof. We proceed by induction on $k=1,2, \ldots, d / 2$. Suppose that $k=1$. We consider each case $1 \in K_{t} \quad(0 \leq t \leq 5)$ separately.

Suppose that $1 \in K_{0}$. For each $\mathfrak{l} \in \Omega_{\alpha}$, set $Y_{1}(\mathfrak{l})=Z_{i_{1}}$, and write $Z_{j\left(i_{1}\right)}=$ $X_{1}+i X_{2}$, where $X_{t} \in \mathfrak{g}$. Set

$$
X_{1}(\mathfrak{l})=\operatorname{Re}\left(\mathfrak{l}\left(\left[\bar{Z}_{j\left(i_{1}\right)}, Y_{1}(\mathfrak{l})\right]\right) Z_{j\left(i_{1}\right)}\right), \quad X_{1}(\mathfrak{l})^{\sim}=\operatorname{Im}\left(\mathfrak{l}\left(\left[\bar{Z}_{j\left(i_{1}\right)}, Y_{1}(\mathfrak{l})\right]\right) Z_{j\left(i_{1}\right)}\right) .
$$

Let $\phi(\mathfrak{l})=1$. Then $\mathfrak{l}\left(\left[X_{1}(\mathfrak{l}), Y_{1}(\mathfrak{l})\right]\right) \neq 0, \mathfrak{l}\left(\left[X_{1}(\mathfrak{l})^{\sim}, Y_{1}(\mathfrak{l})\right]\right)=0$. If $j\left(i_{1}\right) \notin I^{\prime}$, then $j\left(i_{1}\right) \notin I$ and $X_{1}(\mathfrak{l})$ and $X_{1}(\mathfrak{l})^{\sim}$ form a basis of $\mathfrak{s}_{j\left(i_{k}\right)^{\prime \prime}}$ modulo $\mathfrak{s}_{j\left(i_{k}\right)^{\prime}}$. It is immediate from the definition of $\mathfrak{h}_{1}(\mathfrak{l})$ that $\mathfrak{h}_{1}(\mathfrak{l})=\left\{Y_{1}(\mathfrak{l})\right\}^{\mathfrak{l}}$, and hence that $X_{1}(\mathfrak{l})^{\sim} \in \mathfrak{h}_{1}(\mathfrak{l})$.

Suppose next that $1 \in K_{1}$ so that $\bar{Z}_{i_{1}}=Z_{i_{1}+1}$. Fix $\mathfrak{l} \in \Omega_{\alpha}$ and set

$$
Z=Z(\mathfrak{l})=\mathfrak{l}\left(\left[Z_{j\left(i_{1}\right)}, Z_{i_{1}}\right]\right) \bar{Z}_{i_{1}}-\mathfrak{l}\left(\left[Z_{j\left(i_{1}\right)}, \bar{Z}_{i_{1}}\right]\right) Z_{i_{1}}
$$

so that $\mathfrak{l}\left(\left[Z_{j\left(i_{1}\right)}, Z\right]\right)=0$. We claim that $Z \in R(\mathfrak{l})$. For let $c \in \mathbf{C}$ such that $\bar{Z}_{i_{1}}+c Z_{i_{1}}+W \in R(\mathfrak{l}), W \in \mathfrak{s}_{i_{1}-1}$. Then by definition of $i_{1}, W \in R(\mathfrak{l})$ and hence $\bar{Z}_{i_{1}}+c Z_{i_{1}}=Z_{0} \in R(\mathfrak{l})$. It follows that $Z_{0}$ is a multiple of $Z$ and the claim is proved.

Now if $Z_{j\left(i_{1}\right)} \in \mathfrak{g}$ we simply set $X_{1}(\mathfrak{l})=Z_{j\left(i_{1}\right)}, \phi(\mathfrak{l})=1$, and

$$
Y_{1}(\mathfrak{l})=\operatorname{Re}\left(\mathfrak{l}\left(\left[Z_{j\left(i_{1}\right)}, \bar{Z}_{i_{1}}\right]\right) Z_{i_{1}}\right)
$$


It is immediate that $X_{1}(\mathfrak{l}) \in \mathfrak{s}_{j\left(i_{1}\right)} \sim \mathfrak{s}_{j\left(i_{1}\right)^{\prime}}, Y_{1}(\mathfrak{l}) \in \mathfrak{s}_{i_{1}^{\prime \prime}} \sim \mathfrak{s}_{i_{1}^{\prime}}$, and $\mathfrak{h}_{1}(\mathfrak{l})=$ $\left\{Y_{1}(\mathfrak{l})\right\}^{\mathfrak{l}}$. Suppose that $Z_{j\left(i_{1}\right)} \notin \mathfrak{g}$. Note that since $Z \in R(\mathfrak{l})$, then $\bar{Z} \in R(\mathfrak{l})$, and since $Z_{i_{1}} \notin R(\mathfrak{l}), Z$ and $\bar{Z}$ must be linearly dependent. It follows that the complex numbers $\mathfrak{l}\left(\left[Z_{j\left(i_{1}\right)}, \operatorname{Re}\left(Z_{i_{1}}\right)\right]\right)$ and $\mathfrak{l}\left(\left[Z_{j\left(i_{1}\right)}, \operatorname{Im}\left(Z_{i_{1}}\right)\right]\right)$ are R-linearly dependent. For simplicity of notation set $Y_{1}=\operatorname{Re}\left(Z_{i_{1}}\right)$ and $Y_{2}=\operatorname{Im}\left(Z_{i_{1}}\right)$. Define, for $t=1,2$,

$$
O_{t}=\left\{\mathfrak{l} \in \Omega_{\alpha}: \mathfrak{l}\left(\left[Z_{j\left(i_{1}\right)}, Y_{t}\right]\right) \neq 0\right\} .
$$

For $\mathfrak{l} \in O_{t},(t=1,2)$ set $\phi_{t}(\mathfrak{l})=\mathfrak{l}\left(\left[Z_{j\left(i_{1}\right)}, Y_{t}\right]\right) /\left|\mathfrak{l}\left(\left[Z_{j\left(i_{1}\right)}, Y_{t}\right]\right)\right|$, set

$$
Y_{1, t}(\mathfrak{l})=\phi_{l}(\mathfrak{l})^{-1}\left(\mathfrak{l}\left(\left[Z_{j\left(i_{1}\right)}, Y_{1}\right]\right) Y_{1}+\mathfrak{l}\left(\left[Z_{j\left(i_{1}\right)}, Y_{2}\right]\right) Y_{2}\right)
$$

and

If $j\left(i_{1}\right) \notin I^{\prime}$, set

$$
X_{1, t}(\mathfrak{l})=\operatorname{Re}\left(\mathfrak{l}\left(\left[\bar{Z}_{j\left(i_{1}\right)}, Y_{1, t}(\mathfrak{l})\right]\right) Z_{j\left(i_{1}\right)}\right)
$$

$$
X_{1, t}(\mathfrak{l})^{\sim}=\operatorname{Im}\left(\mathfrak{l}\left(\left[\bar{Z}_{j\left(i_{1}\right)}, Y_{1, t}(\mathfrak{l})\right]\right) Z_{j\left(i_{1}\right)}\right) .
$$

It is clear $\mathfrak{l} \rightarrow \phi_{1, t}(\mathfrak{l})$ is analytic on $O_{t}(t=1,2)$ and that $\mathfrak{l} \rightarrow \phi_{1, t}(\mathfrak{l}) X_{1, t}(\mathfrak{l})$, $\mathfrak{l} \rightarrow \phi_{1, t}(\mathfrak{l}) X_{1, t}(\mathfrak{l})^{\sim}, \mathfrak{l} \rightarrow \phi_{1, t}(\mathfrak{l}) Y_{1, t}(\mathfrak{l})$ extend to rational (in fact polynomial) functions on $\Omega_{\alpha}$ independently of $t$. (We drop the subscript $t$ in denoting these functions.) For each $\mathfrak{l} \in \Omega_{\alpha}$, and for each $t=1,2$, we have that $Y_{1, t}(\mathfrak{l}) \in \mathfrak{s}_{i_{1}^{\prime \prime}} \sim \mathfrak{s}_{i_{1}^{\prime}}$, that $X_{1, t}(\mathfrak{l})$ and $X_{1, t}(\mathfrak{l})^{\sim}$ form a basis of $\mathfrak{s}_{j\left(i_{1}\right)^{\prime \prime}}$ modulo $\mathfrak{s}_{j\left(i_{1}\right)^{\prime}}$, and $\mathfrak{l}\left(\left[X_{1, t}(\mathfrak{l}), Y_{1, t}(\mathfrak{l})\right]\right) \neq 0$. It is also immediate that $\mathfrak{h}_{1}(\mathfrak{l})=\left\{Y_{1, t}(\mathfrak{l})\right\}^{\mathfrak{l}}$. Note also that in this case $j\left(i_{1}\right) \notin I$, and hence (if $\left.j\left(i_{1}\right) \notin I^{\prime}\right), X_{1, t}(\mathfrak{l})^{\sim} \in \mathfrak{h}_{1}(\mathfrak{l})$.

A standard argument (using the fact that $\mathfrak{g}$ is exponential) shows that $1 \notin$ $K_{3}$. To finish the case $k=1$, it remains for us to verify the lemma for $k=1 \in K_{4}$. Here we have $A \in L_{i_{1}}(\mathfrak{l}) \sim \operatorname{ker}\left(\gamma_{i_{1}}\right)$. A standard argument shows that $\gamma_{j\left(i_{1}\right)}=-\gamma_{i_{1}}$ and $Z_{j\left(i_{2}\right)}=\bar{Z}_{j\left(i_{1}\right)}$ and that for every $\mathfrak{l} \in \Omega_{\alpha}, \mathfrak{l}\left(\left[Y_{1}, Y_{2}\right]\right)=0$. Set

$$
Y_{1}(\mathfrak{l})=\operatorname{Re}\left(Z_{i_{1}}\right), \quad Y_{2}(\mathfrak{l})=\operatorname{Im}\left(Z_{i_{1}}\right) .
$$

Since $i_{1}+1=i_{2}$ and $Z_{j\left(i_{2}\right)}=\bar{Z}_{j\left(i_{1}\right)}$, we have $\mathfrak{l}\left(\left[Z_{j\left(i_{1}\right)}, Y_{1}(\mathfrak{l})\right]\right) \neq 0$. Set

$$
X_{1}(\mathfrak{l})=\operatorname{Re}\left(\mathfrak{l}\left(\left[\bar{Z}_{j\left(i_{1}\right)}, Y_{1}(\mathfrak{l})\right]\right) Z_{j\left(i_{1}\right)}\right)
$$

and

$$
X_{1}(\mathfrak{l})^{\sim}=\operatorname{Im}\left(\mathfrak{l}\left(\left[\bar{Z}_{j\left(i_{1}\right)}, Y_{1}(\mathfrak{l})\right]\right) Z_{j\left(i_{1}\right)}\right) .
$$

Set

$$
X_{2}(\mathfrak{l})=X_{1}(\mathfrak{l})^{\sim}-w\left(X_{1}(\mathfrak{l})^{\sim}, \mathfrak{l}\right) Y_{1}(\mathfrak{l}),
$$

where $w\left(X_{1}(\mathfrak{l})^{\sim}, \mathfrak{l}\right)=\mathfrak{l}\left(\left[X_{1}(\mathfrak{l})^{\sim}, X_{1}(\mathfrak{l})\right]\right) / \mathfrak{l}\left(\left[Y_{1}(\mathfrak{l}), X_{1}(\mathfrak{l})\right]\right)$. Set $\phi(\mathfrak{l})=1, \mathfrak{l} \in \Omega_{\alpha}$. Then the maps $\mathfrak{l} \rightarrow Y_{1}(\mathfrak{l}), \mathfrak{l} \rightarrow Y_{2}(\mathfrak{l}), \mathfrak{l} \rightarrow X_{1}(\mathfrak{l}), \mathfrak{l} \rightarrow X_{2}(\mathfrak{l})$, and $\mathfrak{l} \rightarrow X_{1}(\mathfrak{l})^{\sim}$ are rational on $\Omega_{\alpha}$ and we have that $\mathfrak{s}_{j\left(i_{1}\right)^{\prime \prime}}=\operatorname{span}\left\{X_{1}(\mathfrak{l}), X_{1}(\mathfrak{l})^{\sim}\right\}+\mathfrak{s}_{j\left(i_{1}\right)^{\prime}}=$ $\operatorname{span}\left\{X_{1}(\mathfrak{l}), X_{2}(\mathfrak{l})\right\}+\mathfrak{s}_{j\left(i_{1}\right)^{\prime}}$. By construction, $\mathfrak{l}\left(\left[X_{1}(\mathfrak{l}), X_{2}(\mathfrak{l})\right]\right)=\mathfrak{l}\left(\left[X_{1}(\mathfrak{l}), Y_{2}(\mathfrak{l})\right]\right)$ $=\mathfrak{l}\left(\left[X_{2}(\mathfrak{l}), Y_{1}(\mathfrak{l})\right]\right)=0$, and by definition of $\mathfrak{h}_{2}(\mathfrak{l}), \mathfrak{h}_{2}(\mathfrak{l})=\left\{Y_{1}(\mathfrak{l}), Y_{2}(\mathfrak{l})\right\}^{\mathfrak{l}}$. This finishes the proof for $k=1$.

Suppose that $k>1$, and suppose that there is a finite cover $F_{0}$ of $\Omega_{\alpha}$ consisting of Zariski open subsets, and having the properties described in the lemma for $1 \leq r \leq k-1$. Thus for each $O \in F_{0}$, and $r, 1 \leq r \leq k-1$, we have functions $Y_{r}$ and $X_{r}$ from $O$ into $\mathfrak{g}$ and $\phi_{r}$ from $O$ into $S^{1}$ satisfying 
the properties set forth in the lemma, and if $r \notin I^{\prime}, r \notin K_{3}$, and $Z_{j\left(i_{r}\right)} \neq Z_{j\left(i_{r^{\prime}}\right)}$, $r^{\prime}<r$ we have the function $X_{r}^{\sim}$ on $O$ as in the lemma.

For each $r, 1 \leq r \leq k-1, \mathfrak{l} \in \Omega_{\alpha}$, set

$$
\mathfrak{k}_{r}(\mathfrak{l})=\operatorname{span}\left\{\phi_{1}(\mathfrak{l}) X_{1}(\mathfrak{l}), \ldots, \phi_{r}(\mathfrak{l}) X_{r}(\mathfrak{l}), \phi_{1}(\mathfrak{l}) Y_{1}(\mathfrak{l}), \ldots, \phi_{r}(\mathfrak{l}) Y_{r}(\mathfrak{l})\right\}
$$

then we have $\mathfrak{g}_{c}=\mathfrak{k}_{r}(\mathfrak{l}) \oplus \mathfrak{k}_{r}(\mathfrak{l})^{\mathfrak{l}}$. For each $Z \in \mathfrak{g}_{c}$ let $\rho_{r}(Z, \mathfrak{l})$ be the projection of $Z$ into $\mathfrak{k}_{r}(\mathfrak{l})^{\mathfrak{l}}$ parallel to $\mathfrak{k}_{r}(\mathfrak{l})$. It follows that for each $Z \in \mathfrak{g}_{c}$, and for each $r, 1 \leq r \leq k-1, \mathfrak{l} \rightarrow \rho_{r}(Z, \mathfrak{l})$ is a rational mapping of $\Omega_{\alpha}$ into $\mathfrak{g}_{c}$. Moreover it is easily seen that for each $\mathfrak{l} \in \Omega_{\alpha}$,

(i) $\rho_{r}(\cdot, \mathfrak{l})$ maps $\mathfrak{g}_{c}$ into $\mathfrak{h}_{r}(\mathfrak{l})$ and commutes with conjugation,

(ii) for each $j=1,2, \cdots, n$ and $\mathfrak{l} \in \Omega_{\alpha}, \rho_{r}\left(\mathfrak{s}_{j}, \mathfrak{l}\right) \subset \mathfrak{s}_{j^{\prime \prime}}$, and

(iii) if $r \notin K_{4}$, and if $j \notin\left\{i_{1}, i_{2}, \ldots, i_{r}, j\left(i_{1}\right), j\left(i_{2}\right), \ldots, j\left(i_{r}\right)\right\}$, then $\rho_{r}\left(Z_{j}, \mathfrak{l}\right) \in \mathfrak{s}_{j} \sim \mathfrak{s}_{j-1}$.

First we prove a fact which will be used several times in the sequel, namely, that for any $r(1 \leq r \leq k)$, if $r \in K_{4}$, then $j\left(i_{r+1}\right)>j\left(i_{r}\right)^{\prime}$. Setting $\rho=$ $\rho_{r-1}(\cdot, l)$, if $j\left(i_{r+1}\right) \leq j\left(i_{r}\right)^{\prime}, \rho\left(Z_{j\left(i_{r+1}\right)}\right) \in \mathfrak{h}_{r-1}(\mathfrak{l}) \cap \mathfrak{s}_{j\left(i_{r}\right)^{\prime}} \subset \mathfrak{h}_{r}(\mathfrak{l}) \cap \mathfrak{s}_{j\left(i_{r}\right)^{\prime}}$, and $\rho\left(Z_{i_{r+1}}\right) \in \mathfrak{h}_{r}(\mathfrak{l}) \cap \mathfrak{s}_{i_{r+1}}$; hence

$$
\mathfrak{l}\left(\left[\rho\left(\bar{Z}_{j\left(i_{r+1}\right)}\right), \rho\left(Z_{i_{r}}\right)\right]\right)=\mathfrak{l}\left(\left[\rho\left(Z_{j\left(i_{r+1}\right)}\right), \rho\left(Z_{i_{r+1}}\right)\right]\right)^{-} \neq 0 .
$$

But $\rho\left(\bar{Z}_{j\left(i_{r+1}\right)}\right) \in \mathfrak{h}_{r-1}(\mathfrak{l}) \cap \mathfrak{s}_{j\left(i_{r}\right)^{\prime}} \subset \mathfrak{h}_{r}(\mathfrak{l})$ implies that $\mathfrak{l}\left(\left[\rho\left(\bar{Z}_{j\left(i_{r+1}\right)}\right), \rho\left(Z_{i_{r}}\right)\right]\right)=0$, a contradiction.

We shall next verify the induction step considering each case $k \in K_{t}, 0 \leq$ $t \leq 5$, separately. We will show that there is a cover $F_{1}$ having the properties described in the lemma for $1 \leq k^{\prime} \leq k$. We shall begin each case by implicitly fixing $\mathfrak{l} \in \Omega_{\alpha}$. When $\mathfrak{l}$ is fixed, we set $\rho=\rho_{k-1}(\cdot, \mathfrak{l})$ if $k \notin K_{5}$ and $\rho=$ $\rho_{k-2}(\cdot, \mathfrak{l})$ if $k \in K_{5}$ for brevity of notation.

Case (0): $k \in K_{0}$. Here we let $F_{1}=F_{0}$. Set $Y_{k}(\mathfrak{l})=\rho\left(Z_{i_{k}}\right)$, and set

$$
X_{k}(\mathfrak{l})=\operatorname{Re}\left(\mathfrak{l}\left(\left[\rho\left(Z_{j\left(i_{k}\right)}\right), Y_{k}(\mathfrak{l})\right]\right) \rho\left(Z_{j\left(i_{k}\right)}\right)\right) \text {. }
$$

Since $\rho\left(Z_{i_{k}}\right)$ is a basis of $\mathfrak{s}_{i_{k}} \cap \mathfrak{h}_{k-1}(\mathfrak{l})$ modulo $\mathfrak{s}_{i_{k}-1} \cap \mathfrak{h}_{k-1}(\mathfrak{l})$, then by definition of $\mathfrak{h}_{k}(\mathfrak{l}), \mathfrak{h}_{k}(\mathfrak{l})=\mathfrak{h}_{k-1}(\mathfrak{l}) \cap\left\{Y_{k}(\mathfrak{l})\right\}^{\mathfrak{l}}$. It follows that $\mathfrak{l}\left(\left[X_{k}(\mathfrak{l}), Y_{k}(\mathfrak{l})\right]\right) \neq 0$. If $j\left(i_{k}\right) \in I^{\prime}$ and $\bar{Z}_{j\left(i_{k}\right)} \neq Z_{j\left(i_{r}\right)}, r<k$, then set

$$
X_{k}(\mathfrak{l})^{\sim}=\operatorname{Im}\left(\mathfrak{l}\left(\left[\rho\left(\bar{Z}_{j\left(i_{k}\right)}\right), Y_{k}(\mathfrak{l})\right]\right) \rho\left(Z_{j\left(i_{k}\right)}\right)\right) .
$$

In this case $\mathfrak{l}\left(\left[X_{k}(\mathfrak{l})^{\sim}, Y_{k}(\mathfrak{l})\right]\right)=0$ and $X_{k}(\mathfrak{l})$ and $X_{k}(\mathfrak{l})^{\sim}$ form a basis of $\mathfrak{s}_{j\left(i_{k}\right)^{\prime \prime}}$ modulo $\mathfrak{s}_{j\left(i_{k}\right)^{\prime}}$. We set $\phi_{k}(\mathfrak{l})=1$ and it is immediate from the rationality of $\rho_{k-1}\left(Z_{j\left(i_{k}\right)}, \cdot\right)$ that the maps $\mathfrak{l} \rightarrow X_{k}(\mathfrak{l})$ and $\mathfrak{l} \rightarrow Y_{k}(\mathfrak{l})$ are rational on $\Omega_{\alpha}$. Let $O \in F_{0}$; since $Y_{k}(\mathfrak{l})$ and $X_{k}(\mathfrak{l})$ lie in the range of $\rho$, we have for each $\mathfrak{l} \in O$,

$$
\mathfrak{l}\left(\left[Y_{k}(\mathfrak{l}), X_{r}(\mathfrak{l})\right]\right)=\mathfrak{l}\left(\left[Y_{k}(\mathfrak{l}), Y_{r}(\mathfrak{l})\right]\right)=0, \quad 1 \leq r \leq k-1 .
$$

Case (1): $k \in K_{1}$. The proof here is similar to the proof in the case $k=1 \in K_{1}$. Set

$$
Z=Z(\mathfrak{l})=\mathfrak{l}\left(\left[\rho\left(Z_{j\left(i_{k}\right)}\right), \rho\left(Z_{i_{k}}\right)\right]\right) \rho\left(\bar{Z}_{i_{k}}\right)-\mathfrak{l}\left(\left[\rho\left(Z_{j\left(i_{k}\right)}\right), \rho\left(\bar{Z}_{i_{k}}\right)\right]\right) \rho\left(Z_{i_{k}}\right)
$$

so that $\mathfrak{l}\left(\left[\rho\left(Z_{j\left(i_{k}\right)}\right), Z\right]\right)=0$. It is easily seen that $Z \in R\left(\mathfrak{l}, \mathfrak{h}_{k-1}(\mathfrak{l})\right)$; the proof of this is similar to the case $k=1 \in K_{1}$ and will be omitted here. 
Now since $\rho\left(\mathfrak{g}_{c}\right) \in \mathfrak{h}_{k-1}(\mathfrak{l}), \mathfrak{l}\left(\left[\rho\left(Z_{j\left(i_{k}\right)}\right), \bar{Z}\right]\right)=\mathfrak{l}\left(\left[\rho\left(Z_{j\left(i_{k}\right)}\right), Z\right]\right)^{-}=0$. It follows (as in the above case $k=1 \in K_{1}$ ) that $Z$ and $\bar{Z}$ must be linearly dependent, and that the numbers

$$
\mathfrak{l}\left(\left[\rho\left(Z_{j\left(i_{k}\right)}\right), \operatorname{Re}\left(\rho\left(Z_{i_{k}}\right)\right)\right]\right) \text { and } \mathfrak{l}\left(\left[\rho\left(Z_{j\left(i_{k}\right)}\right), \operatorname{Im}\left(\rho\left(Z_{i_{k}}\right)\right)\right]\right)
$$

are R-linearly dependent. Now let $O \in F_{0}$; then $O=O_{1} \cup O_{2}$, where $O_{1}=\{\mathfrak{l} \in$ $\left.O: \mathfrak{l}\left(\left[\rho\left(Z_{j\left(i_{k}\right)}\right), \operatorname{Re}\left(\rho\left(Z_{i_{k}}\right)\right)\right]\right) \neq 0\right\}$ and $O_{2}=\left\{\mathfrak{l} \in O: \mathfrak{l}\left(\left[\rho\left(Z_{j\left(i_{k}\right)}\right), \operatorname{Im}\left(\rho\left(Z_{i_{k}}\right)\right)\right]\right) \neq\right.$ $0\}$. For $\mathfrak{l} \in O_{1}$, set

$$
\phi_{k, 1}(\mathfrak{l})=\mathfrak{l}\left(\left[\rho\left(Z_{j\left(i_{k}\right)}\right), \operatorname{Re}\left(\rho\left(Z_{i_{k}}\right)\right)\right]\right) /\left|\mathfrak{l}\left(\left[\rho\left(Z_{j\left(i_{k}\right)}\right), \operatorname{Re}\left(\rho\left(Z_{i_{k}}\right)\right)\right]\right)\right|
$$

and for $\mathfrak{l} \in O_{2}$ set

$$
\phi_{k, 2}(\mathfrak{l})=\mathfrak{l}\left(\left[\rho\left(Z_{j\left(i_{k}\right)}\right), \operatorname{Im}\left(\rho\left(Z_{i_{k}}\right)\right)\right]\right) /\left|\mathfrak{l}\left(\left[\rho\left(Z_{j\left(i_{k}\right)}\right), \operatorname{Im}\left(\rho\left(Z_{i_{k}}\right)\right)\right]\right)\right| .
$$

For $t=1,2, \mathfrak{l} \in O_{t}$, set

$$
\begin{aligned}
Y_{k, t}(\mathfrak{l})= & \phi_{k, t}(\mathfrak{l})^{-1}\left(\mathfrak{l}\left(\left[\rho\left(Z_{j\left(i_{k}\right)}\right), \operatorname{Re}\left(\rho\left(Z_{i_{k}}\right)\right)\right]\right) \operatorname{Re}\left(\rho\left(Z_{i_{k}}\right)\right)\right. \\
& +\mathfrak{l}\left(\left[\rho\left(Z_{j\left(i_{k}\right)}\right), \operatorname{Im}\left(\rho\left(Z_{i_{k}}\right)\right)\right]\right) \operatorname{Im}\left(\rho\left(Z_{i_{k}}\right)\right)
\end{aligned}
$$

and

$$
X_{k, t}(\mathfrak{l})=\operatorname{Re}\left(\mathfrak{l}\left(\left[\rho\left(\bar{Z}_{j\left(i_{k}\right)}\right), Y_{k, t}(\mathfrak{l})\right]\right) \rho\left(Z_{j\left(i_{k}\right)}\right)\right)
$$

If $j\left(i_{k}\right) \notin I^{\prime}$ and $\bar{Z}_{j\left(i_{k}\right)} \neq Z_{j\left(i_{r}\right)}, r<k$, then set

$$
X_{k, 1}(\mathfrak{l})^{\sim}=\operatorname{Im}\left(\mathfrak{l}\left(\left[\rho\left(\bar{Z}_{j\left(i_{k}\right)}\right), Y_{k, t}(\mathfrak{l})\right]\right) \rho\left(Z_{j\left(i_{k}\right)}\right)\right) .
$$

It is immediate from the rationality of $\rho$ that $\mathfrak{l} \rightarrow \phi_{k, t}(\mathfrak{l}) X_{k, t}(\mathfrak{l})$ and $\mathfrak{l} \rightarrow$ $\phi_{k, t}(\mathfrak{l}) Y_{k, t}(\mathfrak{l})$ extend to rational functions on $\Omega_{\alpha}$. By definition of $j\left(i_{k}\right)$ we have $\mathfrak{l}\left(\left[X_{k, t}(\mathfrak{l}), Y_{k, t}(\mathfrak{l})\right]\right) \neq 0$, and by construction $\mathfrak{l}\left(\left[X_{k, t}(\mathfrak{l})^{\sim}, Y_{k, t}(\mathfrak{l})\right]\right)=0$, $\mathfrak{l} \in O_{t}$. Since $Y_{k, t}(\mathfrak{l})$ and $X_{k, t}(\mathfrak{l})$ lie in the image of $\rho$, we have

$$
\mathfrak{l}\left(\left[Y_{k, t}(\mathfrak{l}), X_{r}(\mathfrak{l})\right]\right)=\mathfrak{l}\left(\left[Y_{k, t}(\mathfrak{l}), Y_{r}(\mathfrak{l})\right]\right)=0, \quad 1 \leq r \leq k-1 .
$$

Thus the cover $F_{1}=\left\{O_{1}, O_{2}: O \in F_{0}\right\}$ satisfies the conditions of the lemma for all $k^{\prime}, 1 \leq k^{\prime} \leq k$.

Case (2): $k \in K_{2}$. Let $r<k$ such that $i_{k}-1=j\left(i_{r}\right)$. Fix $O \in F_{0}$ and $\mathfrak{l} \in O$. By induction we have $X_{r}(\mathfrak{l})^{\sim} \in \mathfrak{h}_{r}(\mathfrak{l}) \cap \mathfrak{s}_{i_{k}} \sim \mathfrak{s}_{i_{k}-1}$ and $\phi_{r}(\mathfrak{l}) \in S^{1}$, such that the map $\mathfrak{l} \rightarrow \phi_{r}(\mathfrak{l}) X_{r}(\mathfrak{l})^{\sim}$ is rational, and $\left\{X_{r}(\mathfrak{l}), X_{r}(\mathfrak{l})^{\sim}\right\}$ is basis of $\mathfrak{s}_{i_{k}} \bmod \mathfrak{s}_{i_{k}-1}$. Since $\rho\left(X_{r}(\mathfrak{l})\right)=0$ and $\rho\left(Z_{i_{k}}\right) \in \mathfrak{s}_{i_{k}} \sim \mathfrak{s}_{i_{k-1}}$, we have $\rho\left(X_{r}(\mathfrak{l}) \sim\right) \in \mathfrak{h}_{k-1}(\mathfrak{l}) \cap\left(\mathfrak{s}_{i_{k}} \sim\right.$ $\left.\mathfrak{s}_{i_{k}-1}\right)$. We set $\phi_{k}(\mathfrak{l})=\phi_{r}(\mathfrak{l})$ and $Y_{k}(\mathfrak{l})=\rho\left(X_{r}(\mathfrak{l})^{\sim}\right)$. Then $\mathfrak{l} \rightarrow \phi_{k}(\mathfrak{l}) Y_{k}(\mathfrak{l})$ is rational and for each $\mathfrak{l} \in \Omega_{\alpha}, \mathfrak{h}_{k}(\mathfrak{l})=\mathfrak{h}_{k-1}(\mathfrak{l}) \cap\left(Y_{k}(\mathfrak{l})\right)^{\mathfrak{l}}$. Set

$$
X_{k}(\mathfrak{l})=\operatorname{Re}\left(\mathfrak{l}\left(\left[\rho\left(\bar{Z}_{j\left(i_{k}\right)}\right), Y_{k}(\mathfrak{l})\right]\right) \rho\left(Z_{j\left(i_{k}\right)}\right)\right),
$$

and if $j\left(i_{k}\right) \notin I^{\prime}$ and $\bar{Z}_{j\left(i_{k}\right)} \neq Z_{j\left(i_{r}\right)}$, for all $r<k$, set

$$
X_{k}(\mathfrak{l})^{\sim}=\operatorname{Im}\left(\mathfrak{l}\left(\left[\rho\left(\bar{Z}_{j\left(i_{k}\right)}\right), Y_{k}(\mathfrak{l})\right]\right) \rho\left(Z_{j\left(i_{k}\right)}\right)\right) .
$$

Then $X_{k}(\mathfrak{l}) \in \mathfrak{s}_{j\left(i_{k}\right)} \cap \mathfrak{h}_{k-1}(\mathfrak{l}) \sim \mathfrak{s}_{j\left(i_{k}\right)-1}$, and the mapping $\mathfrak{l} \rightarrow \phi_{k}(\mathfrak{l}) X_{k}(\mathfrak{l})$ extends to a rational function on $\Omega_{\alpha}$. By definition of $j\left(i_{k}\right)$, we have $\mathfrak{l}\left(\left[X_{k}(\mathfrak{l}), Y_{k}(\mathfrak{l})\right]\right) \neq$ 0 , and if $j\left(i_{k}\right) \notin I^{\prime}$ and $\bar{Z}_{j\left(i_{k}\right)} \neq Z_{j\left(i_{r}\right)}$, for all $r<k$, then $\mathfrak{l}\left(\left[X_{k}(\mathfrak{l})^{\sim}, Y_{k}(\mathfrak{l})\right]\right)=$ 0 . Since $X_{k}(\mathfrak{l}), Y_{k}(\mathfrak{l})$ are in the image of $\rho$, we have $\mathfrak{l}\left(\left[Y_{k}(\mathfrak{l}), X_{r}(\mathfrak{l})\right]\right)=$ $\mathfrak{l}\left(\left[Y_{k}(\mathfrak{l}), Y_{r}(\mathfrak{l})\right]\right)=0,1 \leq r \leq k-1$. Thus in this case we take $F_{1}=F_{0}$. 
Case (3): $k \in K_{3}$. We claim that in this case $i_{k}+1=j\left(i_{k}\right)$. Suppose that for some $r<k, j\left(i_{r}\right)=i_{k}+1$. Note that $r \notin K_{3}$. Fix $\mathfrak{l} \in \Omega_{\alpha}$ and fix $O \in F$ such that $\mathfrak{l} \in O$. To prove the claim we consider the three subcases: (a) $r \in K_{0} \cup K_{1} \cup K_{2}$, (b) $r \in K_{4}$, (c) $r \in K_{5}$, deriving a contradiction in each case.

(a) Here we have $r-1 \notin K_{4}$ and $\mathfrak{h}_{r}(\mathfrak{l})=\mathfrak{h}_{r-1}(\mathfrak{l}) \cap\left\{Y_{r}(\mathfrak{l})\right\}^{\mathfrak{l}}$. Set $\rho=\rho_{r-1}(\cdot, \mathfrak{l})$. Now $\rho\left(Z_{j\left(i_{r}\right)}\right) \in \mathfrak{h}_{r-1}(\mathfrak{l}) \cap \mathfrak{h}_{r-1}(\mathfrak{l})^{-}$, and since $i_{k}<j\left(i_{r}\right)$,

$$
\rho\left(Z_{j\left(i_{r}\right)}\right)^{-}=\rho\left(Z_{i_{k}}\right) \in \mathfrak{h}_{r}(\mathfrak{l}) .
$$

Thus $\mathfrak{l}\left(\left[\rho\left(Z_{j\left(i_{r}\right)}\right), Y_{r}(\mathfrak{l})\right]\right)=\mathfrak{l}\left(\left[\rho\left(Z_{i_{k}}\right), Y_{r}(\mathfrak{l})\right]\right)^{-}=0$. Since $\mathfrak{h}_{r}(\mathfrak{l})=\mathfrak{h}_{r-1}(\mathfrak{l}) \cap$ $\left\{Y_{r}(\mathfrak{l})\right\}^{\mathfrak{l}}$ this gives $\rho\left(Z_{j\left(i_{r}\right)}\right) \in \mathfrak{h}_{r}(\mathfrak{l})$. But according to the induction hypothesis, $\rho\left(Z_{j\left(i_{r}\right)}\right) \in \mathfrak{h}_{r-1}(\mathfrak{l}) \cap \mathfrak{s}_{j\left(i_{r}\right)} \sim \mathfrak{s}_{j\left(i_{r}\right)-1}$; hence by definition of $j\left(i_{r}\right), \rho\left(Z_{j\left(i_{r}\right)}\right) \notin \mathfrak{h}_{r}(\mathfrak{l})$.

(b) We have $r-1 \notin K_{4}$. Set $\rho=\rho_{r-1}(\cdot, \mathfrak{l})$. If $i_{k}+1=j\left(i_{r}\right)$ and $r \in K_{4}$, then $\rho\left(Z_{i_{r+1}}\right) \in \mathfrak{h}_{r}(\mathfrak{l}) \cap \mathfrak{s}_{i_{r+1}}, \rho\left(Z_{i_{k}}\right) \in \mathfrak{h}_{r}(\mathfrak{l}) \cap \mathfrak{s}_{i_{k}}$, and hence

$$
\mathfrak{l}\left(\left[\rho\left(Z_{i_{k}}\right), \rho\left(Z_{i_{r+1}}\right)\right]\right)=\mathfrak{l}\left(\left[\rho\left(Z_{j\left(i_{r}\right)}\right), \rho(Z i r)\right]\right)^{-} \neq 0 .
$$

But this implies $j\left(i_{r+1}\right)<i_{k}=j\left(i_{r}\right)^{\prime}+1$, contradicting the fact that $j\left(i_{r+1}\right)>$ $j\left(i_{r}\right)^{\prime}$.

(c) Here $r-1 \in K_{4}, r-2 \notin K_{4}$; set $\rho=\rho_{r-2}(\cdot, \mathfrak{l})$ as in the lemma. We have $j\left(i_{r}\right)>j\left(i_{r-1}\right)^{\prime}$. Since $Z_{j\left(i_{r}\right)} \neq \bar{Z}_{j\left(i_{r-1}\right)}$, it follows that $j\left(i_{r-1}\right)<i_{k}$. Now choose $a, b \in \mathbf{C}$ such that

$$
W=a \rho\left(Z_{i_{k}}\right)+b \rho\left(Z_{j\left(i_{r-1}\right)}\right) \in \mathfrak{h}_{r-1}(\mathfrak{l}) .
$$

Since $W \in \mathfrak{s}_{i_{k}}$ and $i_{k}<j\left(i_{r}\right), W \in \mathfrak{h}_{r}(\mathfrak{l})$. Also $\bar{W} \in \mathfrak{h}_{r-2}(\mathfrak{l})^{-}$, and since $\mathfrak{h}_{r}(\mathfrak{l})=\mathfrak{h}_{r-2}(\mathfrak{l}) \cap\left\{Y_{r-1}(\mathfrak{l}), Y_{r}(\mathfrak{l})\right\}^{\prime}$, we have $\bar{W} \in \mathfrak{h}_{r}(\mathfrak{l})$. But $\bar{W} \in \mathfrak{s}_{j\left(i_{r}\right)} \sim \mathfrak{s}_{j\left(i_{r}\right)^{\prime}}$ and hence

$$
\begin{aligned}
\mathfrak{s}_{j\left(i_{r}\right)} \cap \mathfrak{h}_{r-1}(\mathfrak{l}) & \subset\left(\mathbf{C} \bar{W}+\mathfrak{s}_{j\left(i_{r}\right)-1}\right) \cap \mathfrak{h}_{r-1}(\mathfrak{l})=\mathbf{C} \bar{W}+\left(\mathfrak{s}_{j\left(i_{r}\right)-1} \cap \mathfrak{h}_{r-1}(\mathfrak{l})\right) \\
& \subset \mathbf{C} \bar{W}+\mathfrak{h}_{r}(\mathfrak{l})=\mathfrak{h}_{r}(\mathfrak{l}),
\end{aligned}
$$

contradicting the definition of $j\left(i_{r}\right)$. This proves the claim.

Now let $O \in F_{0}$. For $\mathfrak{l} \in O$, set $Y_{k}(\mathfrak{l})=\operatorname{Im}\left(\rho\left(Z_{i_{k}}\right)\right), X_{k}(\mathfrak{l})=\operatorname{Re}\left(\rho\left(Z_{i_{k}}\right)\right)$, and set $\phi_{k}(\mathfrak{l})=1$. Then it is easily seen that $F_{1}=F_{0}$ satisfies the conditions of the lemma.

Case (4): $k \in K_{4}$. Recall that in this case $j\left(i_{k+1}\right)>j\left(i_{k}\right)^{\prime}$. We consider two subcases: (a) $\bar{Z}_{j\left(i_{k}\right)}=Z_{j\left(i_{k+1}\right)}$, and (b) $\bar{Z}_{j\left(i_{k}\right)} \neq Z_{j\left(i_{k+1}\right)}$.

(a) Here the proof is similar to the proof given above when $k=1$, with $Z_{i_{1}}$, $Z_{j\left(i_{1}\right)}$ replaced by $\rho\left(Z_{i_{k}}\right), \rho\left(Z_{j\left(i_{k}\right)}\right)$, respectively, and using the properties of the mapping $\rho$. We omit the details.

(b) Here $j\left(i_{k+1}\right) \geq j\left(i_{k}\right)^{\prime \prime}$. Set as before

$$
Z=Z(\mathfrak{l})=\mathfrak{l}\left(\left[\rho\left(Z_{j\left(i_{k}\right)}\right), \rho\left(Z_{i_{k}}\right)\right]\right) \rho\left(\bar{Z}_{i_{k}}\right)-\mathfrak{l}\left(\left[\rho\left(Z_{j\left(i_{k}\right)}\right), \rho\left(\bar{Z}_{i_{k}}\right)\right]\right) \rho\left(Z_{i_{k}}\right),
$$

so that $\mathfrak{l}\left(\left[\rho\left(Z_{j\left(i_{k}\right)}, Z\right]\right)=0\right.$. We claim that $\mathfrak{l}\left(\left[\rho\left(\bar{Z}_{j\left(i_{k}\right)}\right), Z\right]\right)=0$. For since $j\left(i_{k+1}\right)>j\left(i_{k}\right)^{\prime \prime}$, there is $c \in \mathbf{C}$ such that $\rho\left(\bar{Z}_{j\left(i_{k}\right)}\right)+c \rho\left(Z_{j\left(i_{k}\right)}\right) \in \mathfrak{h}_{k+1}(\mathfrak{l})$, and hence

$$
\mathfrak{l}\left(\left[\rho\left(\bar{Z}_{j\left(i_{k}\right)}\right)+c \rho\left(Z_{j\left(i_{k}\right)}\right), Z\right]\right)=0 .
$$

Thus $\mathfrak{l}\left(\left[\rho\left(\bar{Z}_{j\left(i_{k}\right)}\right), Z\right]\right)=0$. Now by the above claim,

$$
\left.\mathfrak{l}\left(\rho\left(Z_{j\left(i_{k}\right)}\right), \bar{Z}\right]\right)=\mathfrak{l}\left(\left[\rho\left(\bar{Z}_{j\left(i_{k}\right)}\right), Z\right]\right)^{-}=0 .
$$


As in the previous cases it follows that $Z$ and $\bar{Z}$ must be linearly dependent (since otherwise $\rho\left(Z_{i_{k}}\right) \in \operatorname{span}(\{Z, \bar{Z}\})$ ) and that the numbers

$$
\mathfrak{l}\left(\left[\rho\left(Z_{j\left(i_{k}\right)}\right), \operatorname{Re}\left(\rho\left(Z_{i_{k}}\right)\right)\right]\right) \text { and } \mathfrak{l}\left(\left[\rho\left(Z_{j\left(i_{k}\right)}, \operatorname{Im}\left(\rho\left(Z_{i_{k}}\right)\right)\right]\right)\right.
$$

are R-linearly dependent. Let $O \in F_{0}$. As before we set

$$
O_{1}=\left\{\mathfrak{l} \in O: \mathfrak{l}\left(\left[\rho\left(Z_{j\left(i_{k}\right)}\right), \operatorname{Re}\left(\rho\left(Z_{i_{k}}\right)\right)\right]\right) \neq 0\right\}
$$

and

$$
O_{2}=\left\{\mathfrak{l} \in O: \mathfrak{l}\left(\rho\left(Z_{j\left(i_{k}\right)}\right), \operatorname{Im}\left(\rho\left(Z_{i_{k}}\right)\right) \mathfrak{l}\right) \neq 0\right\} .
$$

For $\mathfrak{l} \in O_{t}$, define $\phi_{k, t}(\mathfrak{l})$ and $Y_{k, t}(\mathfrak{l}), t=1,2$, exactly as in Case (1) $\left(k \in K_{1}\right)$ and set

$$
\begin{aligned}
Y_{k+1, t}(\mathfrak{l})= & \phi_{k, t}(\mathfrak{l})^{-1}\left(\mathfrak{l}\left(\left[\rho\left(Z_{j\left(i_{k}\right)}\right), \operatorname{Im}\left(\rho\left(Z_{i_{k}}\right)\right)\right]\right) \operatorname{Re}\left(\rho\left(Z_{i_{k}}\right)\right)\right. \\
& -\mathfrak{l}\left(\left[\rho\left(Z_{j\left(i_{k}\right)}\right), \operatorname{Re}\left(\rho\left(Z_{i_{k}}\right)\right)\right]\right) \operatorname{Im}\left(\rho\left(Z_{i_{k}}\right)\right) .
\end{aligned}
$$

Then $\mathfrak{h}_{k+1}(\mathfrak{l})=\mathfrak{h}_{k-1}(\mathfrak{l}) \cap\left\{Y_{k, t}(\mathfrak{l}), Y_{k+1, t}(\mathfrak{l})\right\}^{\mathfrak{l}}$. Define $X_{k, t}(\mathfrak{l})$ and $X_{k, t}(\mathfrak{l})^{\sim}$, $t=1,2$, exactly as in Case (1) $\left(k \in K_{1}\right)$. From the rationality of $\rho$ we have that $\mathfrak{l} \rightarrow \phi_{k, t}(\mathfrak{l}) X_{k, t}(\mathfrak{l})$ and $\mathfrak{l} \rightarrow \phi_{k, t}(\mathfrak{l}) Y_{k, t}(\mathfrak{l})$ extend to rational functions on $\Omega_{\alpha}$ independent of $O$. By definition of $j\left(i_{k}\right)$ we have $\left.\mathfrak{l}\left(\left[X_{k, t}(\mathfrak{l}), Y_{k, t}\right)\right]\right) \neq 0$, and by construction $\mathfrak{l}\left(\left[X_{k, t}(\mathfrak{l}), Y_{k+!, t}(\mathfrak{l})\right]\right)=\mathfrak{l}\left(\left[X_{k, t}(\mathfrak{l})^{\sim}, Y_{k, t}(\mathfrak{l})\right]\right)=0$. Now set

$$
\left.\left.X_{k+1, t}(\mathfrak{l})=\operatorname{Re}\left(\mathfrak{l}\left(\left[\rho_{k}\left(Z_{j\left(i_{k+1}\right)}\right), \mathfrak{l}\right)^{-}, Y_{k+1, t}(\mathfrak{l})\right]\right) \rho_{k}\left(Z_{j\left(i_{k+1}\right)}\right), \mathfrak{l}\right)\right) .
$$

If $j\left(i_{k}\right) \notin I^{\prime}$ and $Z_{j\left(i_{k+1}\right)} \neq Z_{j\left(i_{r}\right)}, r<k$, then set

$$
\left.\left.X_{k+1, t}(\mathfrak{l})^{\sim}=\operatorname{Im}\left(\mathfrak{l}\left(\left[\rho_{k}\left(Z_{j\left(i_{k+1}\right)}\right), \mathfrak{l}\right)^{-}, Y_{k+1, t}(\mathfrak{l})\right]\right) \rho_{k}\left(Z_{j\left(i_{k+1}\right)}\right), \mathfrak{l}\right)\right) .
$$

Then by construction, we have $\mathfrak{l}\left(\left[X_{k+1, t}(\mathfrak{l}), Y_{k, t}(\mathfrak{l})\right]\right)=\mathfrak{l}\left(\left[X_{k+1, t}(\mathfrak{l})^{\sim}, Y_{k, t}(\mathfrak{l})\right]\right)=$ $\mathfrak{l}\left(\left[X_{k+1, t}(\mathfrak{l})^{\sim}, Y_{k+1, t}(\mathfrak{l})\right]\right)=0$. By definition of $\left.j\left(i_{k+1}\right), \rho_{k}\left(Z_{j\left(i_{k+1}\right)}\right), \mathfrak{l}\right)$ and $X_{k}(\mathfrak{l})$ span $\mathfrak{h}_{k-1}(\mathfrak{l})$ modulo $\mathfrak{h}_{k+1}(\mathfrak{l})$, and hence $\mathfrak{l}\left(\left[X_{k+1, t}(\mathfrak{l}), Y_{k+1, t}(\mathfrak{l})\right]\right) \neq 0$. Since $Y_{k, t}(\mathfrak{l})$ and $X_{k, t}(\mathfrak{l})$ lie in the image of $\rho$, we have $\mathfrak{l}\left(\left[Y_{k, t}(\mathfrak{l}), X_{r}(\mathfrak{l})\right]\right)=$ $\mathfrak{l}\left(\left[X_{k, t}(\mathfrak{l}), X_{r}(\mathfrak{l})\right]\right)=0,1 \leq r \leq k-1$. Setting $F_{1}=\left\{O_{1}, O_{2}: O \in F_{0}\right\}, F_{1}$ satisfies the lemma for all $k^{\prime}, 1 \leq k^{\prime} \leq k+1$. Q.E.D.

For each $k, 1 \leq k \leq d, \mathfrak{l} \in \Omega_{\alpha}$, set

$$
\mathfrak{k}_{k}(\mathfrak{l})=\operatorname{span}\left\{\phi_{1}(\mathfrak{l}) X_{1}(\mathfrak{l}), \ldots, \phi_{k}(\mathfrak{l}) X_{k}(\mathfrak{l}), \phi_{1}(\mathfrak{l}) Y_{1}(\mathfrak{l}), \ldots, \phi_{k}(\mathfrak{l}) Y_{k}(\mathfrak{l})\right\}
$$

as in the above proof. Then $\mathfrak{g}_{c}=\mathfrak{k}_{k}(\mathfrak{l}) \oplus \mathfrak{k}_{k}(\mathfrak{l})^{\mathfrak{l}}$. For each $Z \in \mathfrak{g}_{c}$ let $\rho_{k}(Z, \mathfrak{l})$ be the projection of $Z$ into $\mathfrak{k}_{k}(\mathfrak{l})^{\mathfrak{l}}$ parallel to $\mathfrak{k}_{k}(\mathfrak{l})$. The mapping $\rho_{k}: \mathfrak{g}_{c} \times \Omega_{\alpha} \rightarrow \mathfrak{g}_{c}$ is rational, and it is easily seen that for each $\mathfrak{l} \in \Omega_{\alpha}$.

(i) $\rho_{k}(\cdot, \mathfrak{l})$ maps $\mathfrak{g}_{c}$ into $\mathfrak{h}_{k}(\mathfrak{l})$ and commutes with conjugation,

(ii) for each $j=1,2, \ldots, n$ and $\mathfrak{l} \in \Omega_{\alpha}, \rho_{k}\left(\mathfrak{s}_{j}, \mathfrak{l}\right) \subset \mathfrak{s}_{j^{\prime \prime}}$, and

(iii) if $k \in K_{4}$, and if $j \notin\left\{i_{1}, i_{2}, \ldots, i_{k}, j\left(i_{1}\right), j\left(i_{2}\right), \ldots, j\left(i_{k}\right)\right\}$, then $\rho_{k}\left(Z_{j}, \mathfrak{l}\right) \in \mathfrak{s}_{j} \sim \mathfrak{s}_{j-1}$.

The following corollary is immediate.

Corollary 1.4. For each $O \in F$, there are analytic functions $X_{k}$ and $Y_{k} \quad(1 \leq$ $k \leq d / 2)$ from $O$ into $\mathfrak{g}$, such that for each $\mathfrak{l} \in O$, the set $\left\{X_{1}(\mathfrak{l}), \ldots, X_{d / 2}(\mathfrak{l})\right.$, $\left.Y_{1}(\mathfrak{l}), \ldots, Y_{d / 2}(\mathfrak{l})\right\}$ is a basis of $\mathfrak{g}$ modulo $R(\mathfrak{l})$, and

$$
\mathfrak{l}\left(\left[X_{r}(\mathfrak{l}), X_{s}(\mathfrak{l})\right]\right)=\mathfrak{l}\left(\left[Y_{r}(\mathfrak{l}), Y_{s}(\mathfrak{l})\right]\right)=0, \quad(1 \leq r, s \leq d / 2),
$$

$\mathfrak{l}\left(\left[X_{r}(\mathfrak{l}), Y_{s}(\mathfrak{l})\right]\right)=0, r \neq s$, and $\mathfrak{l}\left(\left[X_{k}(\mathfrak{l}), Y_{k}(\mathfrak{l})\right]\right) \neq 0,(1 \leq k \leq d / 2)$.

We can now obtain for each $j \in J, \mathfrak{l} \in \Omega_{\alpha}$, the basis of $L_{j^{\prime}}(\mathfrak{l}) / L_{j^{\prime \prime}}(\mathfrak{l})$ which varies analytically with $\mathfrak{l} \in O$, generalizing Lemma 1.1 of [4]. 
Proposition 1.5. Fix $O \in F$. For each $j \in J$, there are analytic functions $r_{j}: O \rightarrow \mathfrak{g}$, and $z_{j}: O \rightarrow S^{1}$, such that for each $\mathfrak{l} \in O$,

$$
\left[r_{j}(\mathfrak{l}), \mathfrak{l}\right]=z_{j}(\mathfrak{l}) f_{j} \bmod \left(V_{j^{\prime \prime}}\right), \quad \text { if } j \in I^{\prime},
$$

and

$$
\left[r_{j}(\mathfrak{l}), \mathfrak{l}\right]=2 \operatorname{Re}\left(z_{j}(\mathfrak{l}) f_{j}\right) \bmod \left(V_{j^{\prime \prime}}\right), \quad \text { if } j \notin I^{\prime} .
$$

Moreover, if $\tau_{j}=1$ or $\tau_{j}=2$ then $\gamma_{j}\left(r_{j}(\mathfrak{l})\right)=0$ and if $j \notin I$ and

$$
\operatorname{dim}\left(L_{j^{\prime}}(\mathfrak{l}) / L_{j^{\prime \prime}}(\mathfrak{l})\right)=2,
$$

then the numbers $z_{j}(\mathfrak{l})$ and $z_{j+1}(\mathfrak{l})$ are $\mathbf{R}$-linearly independent.

Proof. First, note that by Lemma 1.3, we have for any $i \in I, \mathfrak{l} \in O$,

$$
\mathfrak{s}_{i} \subset R(\mathfrak{l})+\operatorname{span}_{\mathbf{C}}\left(\left\{Y_{r}(\mathfrak{l}): i_{r} \leq i\right\}\right)+\operatorname{span}_{\mathbf{C}}\left(\left\{X_{r}(\mathfrak{l}): j\left(i_{r}\right) \leq i\right\}\right) .
$$

Now let $j \in J$; then for some $k(1 \leq k \leq d / 2) j=i_{k}$ or $j=j\left(i_{k}\right)$. Suppose that $j=i_{k}$. By the above and the properties of the indices $i_{1}, \ldots, i_{d / 2}$, $j\left(i_{1}\right), \ldots, j\left(i_{d / 2}\right)$, we have

$$
\mathfrak{s}_{i_{k}^{\prime}} \subset R(\mathfrak{l})+\operatorname{span}_{\mathbf{C}}\left(\left\{X_{1}(\mathfrak{l}), \ldots, X_{k-1}(\mathfrak{l}), Y_{1}(\mathfrak{l}), \ldots, Y_{k-1}(\mathfrak{l})\right\}\right)
$$

Hence by Corollary 1.4, $X_{k}(\mathfrak{l}) \in L_{i_{k}^{\prime}}(\mathfrak{l})$. On the other hand, since $Y_{k}(\mathfrak{l}) \in \mathfrak{s}_{i_{k}^{\prime \prime}} \sim$ $\mathfrak{s}_{i_{k}^{\prime}}$, we have $X_{k}(\mathfrak{l}) \notin L_{i_{k}}(\mathfrak{l})$ and $\mathfrak{l}\left(\left[Y_{k}(\mathfrak{l}), X_{k}(\mathfrak{l})\right]\right) \neq 0$. Set

$$
r_{j}(\mathfrak{l})=X_{k}(\mathfrak{l}) /\left|\mathfrak{l}\left(\left[Z_{i_{k}}, X_{k}(\mathfrak{l})\right]\right)\right|, z_{j}(\mathfrak{l})=\mathfrak{l}\left(\left[Z_{i_{k}}, X_{k}(\mathfrak{l})\right]\right) /\left|\mathfrak{l}\left(\left[Z_{i_{k}}, X_{k}(\mathfrak{l})\right]\right)\right| .
$$

Suppose next that $j=j\left(i_{k}\right)$. Then

$$
\mathfrak{s}_{j\left(i_{k}\right)^{\prime}} \subset R(\mathfrak{l})+\operatorname{span}_{\mathbf{C}}\left(\left\{Y_{1}(\mathfrak{l}), \ldots, Y_{d / 2}(\mathfrak{l})\right\}\right)+\operatorname{span}_{\mathbf{C}}\left(\left\{X_{r}(\mathfrak{l}): r \neq k\right\}\right),
$$

and hence $Y_{k}(\mathfrak{l}) \in L_{j\left(i_{k}\right)^{\prime}}(\mathfrak{l})$. Since $X_{k}(\mathfrak{l}) \in \mathfrak{s}_{j\left(i_{k}\right)^{\prime \prime}} \sim \mathfrak{s}_{j\left(i_{k}\right)^{\prime}}$, it follows that $Y_{k}(\mathfrak{l}) \notin L_{j\left(i_{k}\right)}(\mathfrak{l})$. Now set

$$
r_{j}(\mathfrak{l})=Y_{k}(\mathfrak{l}) /\left|\mathfrak{l}\left(\left[Z_{j}, Y_{k}(\mathfrak{l})\right]\right)\right|, z_{j}(\mathfrak{l})=\mathfrak{l}\left(\left[Z_{j}, Y_{k}(\mathfrak{l})\right]\right) /\left|\mathfrak{l}\left(\left[Z_{j}, Y_{k}(\mathfrak{l})\right]\right)\right| .
$$

It is clear that if $j \in I^{\prime},\left[r_{j}(\mathfrak{l}), \mathfrak{l}\right]=z_{j}(\mathfrak{l}) f_{j} \bmod \left(V_{j^{\prime \prime}}\right)$, and if $j \notin I^{\prime},\left[r_{j}(\mathfrak{l}), \mathfrak{l}\right]=$ $2 \operatorname{Re}\left(z_{j}(\mathfrak{l}) f_{j}\right) \bmod \left(V_{j^{\prime \prime}}\right)$.

Now let $j \in J$ such that $\tau_{j}=1$. We consider the case $j \notin I$ and $j+1 \in J$, and show that $\gamma_{j}\left(r_{j}(\mathfrak{l})\right)=\gamma_{j}\left(r_{j+1}(\mathfrak{l})\right)=0$, since otherwise $j \in I^{\prime}$ and the proof that $\gamma_{j}\left(r_{j}(\mathfrak{l})=0\right.$ is a simplification of the proof in this case. Fix $\mathfrak{l} \in O$. By Proposition 5 of [17], there is a basis $\left\{X_{1}, X_{2}\right\}$ of $L_{j^{\prime}}(\mathfrak{l})$ modulo $L_{j^{\prime \prime}}(\mathfrak{l})$ consisting of elements belonging to the kernel $C_{j}$ of $\gamma_{j}$. Since $i_{k}<j\left(i_{k}\right)$ $(1 \leq k \leq d / 2)$, the only nontrivial cases are: (1) $j=i_{k}, j+1=i_{k+1}$, and (2) $j+1=i_{k}, j=j\left(i_{r}\right)$. We give the proof for (1); the proof in the other case is similar.

In (1), we have $L_{j^{\prime}}(\mathfrak{l}) \subset \mathfrak{h}_{k-1}(\mathfrak{l})$, and we have $Y_{1}(\mathfrak{l}), Y_{2}(\mathfrak{l}) \in \mathfrak{s}_{j^{\prime \prime}} \sim \mathfrak{s}_{j^{\prime}}$, such that $\mathfrak{h}_{k}(\mathfrak{l})=\mathfrak{h}_{k-1}(\mathfrak{l}) \cap\left\{Y_{1}(\mathfrak{l}), Y_{2}(\mathfrak{l})\right\}^{\mathfrak{l}}$. There are real numbers $b$, and $c$ such that $A=r_{j}(\mathfrak{l})+b X_{1}+c X_{2} \in L_{j^{\prime \prime}}(\mathfrak{l})$. Now

$$
\mathfrak{l}\left(\left[Z_{j},\left[A, X_{1}\right]\right]\right)=\mathfrak{l}\left(\left[\left[Z_{j}, A\right] X_{1}\right]\right)+\mathfrak{l}\left(\left[A,\left[Z_{j}, X_{1}\right]\right]\right)=-\gamma_{j}(A) \mathfrak{l}\left(\left[Z_{j}, X_{1}\right]\right) .
$$

If $\gamma_{j}(A)=0$, then $\gamma_{j}\left(r_{j}(\mathfrak{l})=0\right.$ and we are done. Otherwise $\left[A, X_{1}\right] \in \mathfrak{h}_{k-1}(\mathfrak{l}) \sim$ $\mathfrak{h}_{k}(\mathfrak{l})$. Since $\left[A, X_{1}\right] \in \mathfrak{g}_{p}=\operatorname{nilrad}(\mathfrak{g})$, we have $\mathfrak{g}_{p} \cap \mathfrak{h}_{k-1}(\mathfrak{l}) \not \subset \mathfrak{h}_{k}(\mathfrak{l})$, which implies $j\left(i_{k}\right) \leq p$ and $\mathfrak{s}_{j\left(i_{k}\right)^{\prime \prime}} \subset \mathfrak{g}_{p}$. But by Lemma $1.3, r_{j}(\mathfrak{l}) \in \mathfrak{s}_{j\left(i_{k}\right)^{\prime \prime}}$ and thus $\gamma_{j}\left(r_{j}(\mathfrak{l})\right)=0$. Similarly $\gamma_{j}\left(r_{j+1}(\mathfrak{l})\right)=0$. 
Now choose complex numbers $a$ and $b$ such that

$$
Z_{i_{k}}=a Y_{k}(\mathfrak{l})+b Y_{k+1}(\mathfrak{l}) \text { modulo } \mathfrak{s}_{i_{k}^{\prime}} .
$$

Since $Z_{i_{k}+1}=\bar{Z}_{i_{k}}, a$ and $b$ must be R-linearly independent. Now since $X_{k}(\mathfrak{l}) \in L_{i_{k}^{\prime}}(\mathfrak{l})$ and $X_{k+1}(\mathfrak{l}) \in L_{i_{k}+1^{\prime}}(\mathfrak{l})=L_{i_{k}^{\prime}}(\mathfrak{l})$, then

$$
\begin{aligned}
\mathfrak{l}\left(\left[Z_{i_{k}}, X_{k}(\mathfrak{l})\right]\right) & =\operatorname{ar}\left(\left[Y_{k}(\mathfrak{l}), X_{k}(\mathfrak{l})\right]\right) \quad \text { and } \\
\mathfrak{l}\left(\left[Z_{i_{k}+1}, X_{k+1}(\mathfrak{l})\right]\right) & =\bar{b} \mathfrak{r}\left(\left[Y_{k+1}(\mathfrak{l}), X_{k+1}(\mathfrak{l})\right]\right) .
\end{aligned}
$$

Hence $z_{j}(\mathfrak{l})= \pm a /|a|$ and $z_{j+1}(\mathfrak{l})= \pm \bar{b} /|b|$.

Now suppose that $\tau_{j}=2$. We may assume $j=i_{k}$ (for some $k, 1 \leq k \leq$ $d / 2)$. Then $\operatorname{dim}\left(L_{j^{\prime}}(\mathfrak{l}) / L_{j^{\prime \prime}}(\mathfrak{l})\right)=1$, and there is $X \in C_{j}$ which forms a basis of $L_{j^{\prime}}(\mathfrak{l}) \bmod \left(L_{j^{\prime \prime}}(\mathfrak{l})\right)$. There is a complex number $b$ such that $A=r_{j}(\mathfrak{l})+b X \in$ $L_{j^{\prime \prime}}(\mathfrak{l})$. Now an argument similar to the above shows that either $\gamma_{j}(A)=0$ or $j\left(i_{k}\right) \leq p$, and in either case $\gamma_{j}\left(r_{j}(\mathfrak{l})\right)=0$. Q.E.D.

Since $i_{k}<j\left(i_{k}\right) \quad(1 \leq k \leq d / 2)$, the following corollary follows immediately from the definition of $r_{j\left(i_{k}\right)}$.

Corollary 1.6. For each $k=1,2, \ldots, d / 2, \tau_{j\left(i_{k}\right)}=1$ or 2 .

Let us sum up what we have shown thus far with regard to the functions $\tau_{j}$ $(1 \leq j \leq n)$ on $\Omega_{\alpha}$. For each $j(1 \leq j \leq n)$, if $j \notin J$, if $j=j\left(i_{k}\right)$, for some $k(1 \leq k \leq d / 2)$, or if $j=i_{k}$ for some $k \in K_{2} \cup K_{3} \cup K_{4} \cup K_{5}$, then $\tau_{j}$ is constant on $\Omega_{\alpha}$. Now let $k \in K_{0} \cup K_{1}$, let $i=i_{k}$ and $j=j\left(i_{k}\right)$.

Lemma 1.7. Let $\mathfrak{l} \in \Omega_{\alpha}$, and let $\mathfrak{h}_{1} \supset \mathfrak{h}_{2} \supset \cdots \supset \mathfrak{h}_{d / 2}$ be the polarizing sequence for $\mathfrak{l}$. Then $\tau_{i}(\mathfrak{l})=3$ if and only if $\mathfrak{s}_{j} \cap \mathfrak{h}_{k-1} \cap C_{i}=\mathfrak{s}_{j-1} \cap \mathfrak{h}_{k-1} \cap C_{i}$.

Proof. Recall that $\operatorname{dim}\left(L_{i^{\prime}}(\mathfrak{l}) / L_{i^{\prime \prime}}(\mathfrak{l})\right)=1$. Assume that $\mathfrak{s}_{j} \cap \mathfrak{h}_{k-1} \cap C_{i} \neq \mathfrak{s}_{j-1} \cap$ $\mathfrak{h}_{k-1} \cap C_{i}$, and let $Z \in \mathfrak{s}_{j} \cap \mathfrak{h}_{k-1} \cap C_{i} \sim \mathfrak{s}_{j-1}$. Set $Z^{\prime}=\rho_{k-1}(Z, \mathfrak{l})$; then by Lemma 1.3, $Z^{\prime} \in \mathfrak{s}_{j} \cap L_{i^{\prime}}(\mathfrak{l})$, and since $Z \in \mathfrak{h}_{k-1}$, it follows from the inductive definition of $\rho_{k-1}$ that $Z^{\prime}=Z \bmod \left(\mathfrak{s}_{i-1}\right)$. (For if $r<k-1, r \notin K_{4}$, and $\rho_{r}(Z, \mathfrak{l})=Z \bmod \left(\mathfrak{s}_{i-1}\right)$, then if $r+1 \notin K_{4}$, we have $Y_{r+1}(\mathfrak{l}) \in \mathfrak{s}_{i-1}$ and $\mathfrak{l}\left(\left[Z, Y_{r+1}(\mathfrak{l})\right]\right)=0$, hence

$$
\rho_{r+1}(Z, \mathfrak{l})=\rho_{r}(Z, \mathfrak{l})-\left(\mathfrak{l}\left(\left[Z, X_{r+1}(\mathfrak{l})\right]\right) / \mathfrak{l}\left(\left[Y_{r+1}(\mathfrak{l}), X_{r+1}(\mathfrak{l})\right]\right)\right) Y_{r+1}(\mathfrak{l})
$$

so $\rho_{r+1}(Z, \mathfrak{l})=\rho_{r}(Z, \mathfrak{l})=Z \bmod \left(\mathfrak{s}_{i-1}\right)$. If $r+1 \notin K_{4}$, then similarly $\rho_{r+2}(Z, \mathfrak{l})=Z \bmod \left(\mathfrak{s}_{i-1}\right)$.) Hence $Z^{\prime} \in C_{i}$. Since $Z^{\prime} \in \mathfrak{s}_{j} \cap \mathfrak{h}_{k-1} \sim \mathfrak{s}_{j-1}$, $Z^{\prime} \notin \mathfrak{h}_{k}$ and hence $Z^{\prime} \notin L_{i^{\prime \prime}}(\mathfrak{l})$. We now have (by definition) that $\tau_{i}(\mathfrak{l})=1$ or 2.

For the converse, assume that $\mathfrak{s}_{j} \cap \mathfrak{h}_{k-1} \cap C_{i}=\mathfrak{s}_{j-1} \cap \mathfrak{h}_{k-1} \cap C_{i}$; we will show that $L_{i^{\prime}}(\mathfrak{l}) \cap C_{i}=L_{i^{\prime \prime}}(\mathfrak{l}) \cap C_{i}$. By definition of $j, \mathfrak{s}_{j-1} \cap \mathfrak{h}_{k-1} \subset \mathfrak{h}_{k}$, hence $\mathfrak{s}_{j} \cap \mathfrak{h}_{k-1} \cap C_{i} \subset \mathfrak{h}_{k} \cap C_{i}$. We show first that $\mathfrak{h}_{k} \subset C_{i}$. Let $X=\rho_{k-1}\left(Z_{j}, \mathfrak{l}\right)$, $Y=\rho_{k-1}\left(Z_{i}, \mathfrak{l}\right)$, and let $Z \in \mathfrak{h}_{k}$. We have

$$
[Z, X] \in \mathfrak{s}_{j} \cap \mathfrak{h}_{k-1} \cap C_{i} \subset \mathfrak{h}_{k},
$$

and hence $\mathfrak{l}([[Z, X], Y])=0$. On the other hand since $Z \in \mathfrak{h}_{k}$, we have $\mathfrak{l}([Z,[X, Y]])=0$. Hence

$$
\gamma_{i}(Z) \mathfrak{l}([X, Y])=\mathfrak{l}([X,[Z, Y]])=0
$$


by the Jacobi identity. Thus $\mathfrak{h}_{k} \subset C_{i}$. By the hypothesis, $\mathfrak{h}_{k-1} \not \subset C_{i}$, hence $\mathfrak{h}_{k-1} \cap C_{i}=\mathfrak{h}_{k}$. Since $L_{i^{\prime}}(\mathfrak{l}) \subset \mathfrak{h}_{k-1}$ and $L_{i^{\prime}}(\mathfrak{l}) \cap \mathfrak{h}_{k} \subset L_{i^{\prime \prime}}(\mathfrak{l})$, it follows that $L_{i^{\prime}}(\mathfrak{l}) \cap C_{i}=L_{i^{\prime \prime}}(\mathfrak{l}) \cap C_{i}$. This completes the proof. Q.E.D.

The following generalizes Proposition 1.3 of [4].

Proposition 1.8. We have $\tau_{i}(\mathfrak{l})=3$ if and only if $\gamma_{i}\left(X_{k}(\mathfrak{l})\right) \neq 0$.

Proof. Suppose that $\gamma_{i}\left(X_{k}(\mathfrak{l})\right)=0$. We claim that $C_{i} \supset \mathfrak{s}_{j-1} \cap \mathfrak{h}_{k-1}$. Let $Z \in \mathfrak{s}_{j-1} \cap \mathfrak{h}_{k-1}$. Since $\mathfrak{h}_{k} \supset \mathfrak{s}_{j} \cap \mathfrak{h}_{k-1}$,

$$
\begin{aligned}
\gamma_{i}(Z) \mathfrak{l}\left(\left[X_{k}(\mathfrak{l}), Y_{k}(\mathfrak{l})\right]\right) & =\mathfrak{l}\left(\left[X_{k}(\mathfrak{l}),\left[Z, Y_{k}(\mathfrak{l})\right]\right]\right) \\
& =\mathfrak{l}\left(\left[\left[X_{k}(\mathfrak{l}), Z\right], Y_{k}(\mathfrak{l})\right]\right)+\mathfrak{l}\left(\left[Z,\left[X_{k}(\mathfrak{l}), Y_{k}(\mathfrak{l})\right]\right]\right)=0,
\end{aligned}
$$

and the claim follows. The hypothesis now implies that

$$
\mathfrak{s}_{j} \cap \mathfrak{h}_{k-1} \cap C_{i} \neq \mathfrak{s}_{j-1} \cap \mathfrak{h}_{k-1}=\mathfrak{s}_{j-1} \cap \mathfrak{h}_{k-1} \cap C_{i} .
$$

By Lemma 1.7, $\tau_{i}(\mathfrak{l}) \neq 3$. As for the converse, since

$$
\mathfrak{s}_{i-1} \subset R(\mathfrak{l})+\operatorname{span}_{\mathbf{C}}\left(\left\{Y_{1}(\mathfrak{l}), Y_{2}(\mathfrak{l}), \ldots, Y_{k-1}(\mathfrak{l}), X_{1}(\mathfrak{l}), X_{2}(\mathfrak{l}), \ldots, X_{k-1}(\mathfrak{l})\right\}\right)
$$

it follows that $X_{k}(\mathfrak{l}) \in L_{i-1}(\mathfrak{l}) \sim L_{i}(\mathfrak{l})$. Q.E.D.

We now complete the definition of the layering $\wp$. Let $J \in E$, and $\alpha \in A_{J}$. Consider the set $\varphi_{\alpha}$ of all $j \in J$ such that $\tau_{j}$ is not constant on $\Omega_{\alpha}$. For each $\mathfrak{l} \in \Omega_{\alpha}$, let

$$
\varphi_{\alpha}(\mathfrak{l})=\left\{j \in \varphi_{\alpha}: \tau_{j}(\mathfrak{l})=3\right\} .
$$

Let $\vartheta_{\alpha}=\left\{\varphi_{\alpha}(\mathfrak{l}): \mathfrak{l} \in \Omega_{\alpha}\right\}$ and for each $\varphi \in \vartheta_{\alpha}$, let

$$
\Omega_{\alpha, \varphi}=\left\{\mathfrak{l} \in \Omega_{\alpha}: \varphi_{\alpha}(\mathfrak{l})=\varphi\right\} .
$$

Let $\varphi \in \vartheta_{\alpha}$, and let $j \in \varphi$. Then $j=i_{k}$, for some $k \quad(1 \leq k \leq d / 2)$. By Lemma 1.3, we have a rational function $\xi_{j}: \Omega_{\alpha} \rightarrow \mathfrak{g}_{c}$ such that for each $O \in F, \xi_{j}(\mathfrak{l})=\phi_{k}(\mathfrak{l}) X_{k}(\mathfrak{l}), \mathfrak{l} \in O$. Set

$$
q_{j}(\mathfrak{l})=\gamma_{j}\left(\xi_{j}(\mathfrak{l})\right) / \mathfrak{l}\left(\left[Z_{j}, \xi_{j}(\mathfrak{l})\right]\right) .
$$

By Proposition 1.8 we have $\tau_{j}(\mathfrak{l})=3$ if and only if $q_{j}(\mathfrak{l}) \neq 0$. Thus $\Omega_{\alpha, \varphi}$ is an algebraic subset of $\Omega_{\alpha}$. Note that if $O \in F, \mathfrak{l} \in O$, and $r_{j}(\mathfrak{l}), z_{j}(\mathfrak{l})$ are as in Proposition 1.5, then

$$
q_{j}(\mathfrak{l})=z_{j}(\mathfrak{l})^{-1} \gamma_{j}\left(r_{j}(\mathfrak{l})\right)
$$

Let

$$
\wp=\left\{\Omega_{\alpha, \varphi}: \varphi \in \vartheta_{\alpha}, \alpha \in A_{J}, J \in \mathfrak{j}\right\} ;
$$

each $\Omega \in \wp$ is a $G$-invariant algebraic subset of $\mathfrak{g}^{*}$. For each $\Omega \in \wp$, the functions $\tau_{j}$ are constant on $\Omega$. The layering $\wp$ also has the following desirable property.

Proposition 1.9. There is an ordering $\Omega_{1}<\Omega_{2}<\cdots<\Omega_{u}$ of $\wp$ such that for each $\Omega, \cup\left\{\boldsymbol{\Omega}^{\prime}: \boldsymbol{\Omega}^{\prime} \leq \boldsymbol{\Omega}\right\}$ is a Zariski-open subset of $\mathfrak{g}^{*}$.

Proof. The proof here is a repetition of the proof of Proposition 1.4 of [4]. Q.E.D. 


\section{THE COLLECTIVE ORBIT STRUCTURE}

Let $G$ be a connected, simply connected exponential Lie group with Lie algebra $\mathfrak{g}$, let $\left\{Z_{1}, Z_{2}, \ldots, Z_{n}\right\}$ be a Jordan-Hölder basis for $\mathfrak{g}_{c}$ which corresponds to a good basis of $\mathfrak{g}$, and let $\mathfrak{g}_{c}=\mathfrak{s}_{n} \supset \mathfrak{s}_{n-1} \supset \cdots \supset \mathfrak{s}_{0}=0$ be the corresponding Jordan-Hölder sequence for $\mathfrak{g}_{c}$. Let $\wp$ be the partition of $\mathfrak{g}^{*}$ constructed in $\S 1$. In this section we compute, for each layer $\Omega \in \wp$, a crosssection $\Sigma \subset \Omega$ by proving a result (Proposition 2.5) analogous to Proposition 1.1 , Chapter 2 of [18]. Thus $\Sigma$ is the image of a $G$-invariant analytic function $P: \Omega \rightarrow \Omega$. We then show that $\Sigma$ is in fact algebraic, and that when $\Omega$ is the minimal (Zariski open) layer in $\wp$, that $\Sigma$ is a submanifold of $\mathfrak{g}^{*}$.

Fix $\Omega \in \wp, \Omega=\Omega_{\alpha, \varphi^{\prime}}$, with $J \in \mathfrak{j}$ such that $\alpha \in A_{J}$. For each $j$, the function $\tau_{j}$ is constant on $\Omega$. Define $l=\left\{j \in J: \tau_{j}=2\right\}$ and $\varphi=\{j \in J$ : $\left.\tau_{j}=3\right\}$. Note that $\varphi^{\prime} \subset \varphi$. Let $j \in \varphi$. Define the complex-valued nonsingular rational function $q_{j}$ as in $\S 1$; as before we have the formula

$$
q_{j}(\mathfrak{l})=z_{j}(\mathfrak{l})^{-1} \gamma_{j}\left(r_{j}(\mathfrak{l})\right) \text {. }
$$

The point of this first lemma is that for $j \in \varphi, \mathfrak{l} \in \Omega$, the value $\mathfrak{l}_{j}$ of $\mathfrak{l}$ at $Z_{j}$ is determined solely by $\pi_{j-1}(\mathfrak{l})$ and the value of $q_{j}(\mathfrak{l})$.

Lemma 2.1. Let $\mathfrak{l} \in \Omega$, and let $j \in \varphi$. Then for any $A \in \mathfrak{g}_{c}$ such that $[A, \mathfrak{l}]=$ $f_{j} \bmod \left(V_{j}\right), \gamma_{j}(A)=q_{j}(\mathfrak{l})$. Moreover, for any $\mathfrak{l}^{\prime} \in G \mathfrak{l}$ such that $\pi_{j-1}(\mathfrak{l})=$ $\pi_{j-1}\left(\mathfrak{l}^{\prime}\right)$, we have

$$
\mathfrak{l}_{j}^{\prime}-q_{j}\left(\mathfrak{l}^{\prime}\right)^{-1}=\mathfrak{l}_{j}-q_{j}(\mathfrak{l})^{-1} .
$$

Proof. Let $O \in F$ such that $\mathfrak{l} \in O$ and let $r_{j}(\mathfrak{l})$ and $z_{j}(\mathfrak{l})$ as in Proposition 1.5. Suppose that for some $A \in \mathfrak{g}_{c}$ we have $[A, \mathfrak{l}]=f_{j} \bmod \left(V_{j}\right)$ and $\gamma_{j}(A) \neq q_{j}(\mathfrak{l})$. Set

$$
B=\left(\gamma_{j}\left(r_{j}(\mathfrak{l})\right) A-\gamma_{j}(A) r_{j}(\mathfrak{l})\right) /\left(z_{j}(\mathfrak{l}) \gamma_{j}(A)-\gamma_{j}\left(r_{j}(\mathfrak{l})\right)\right) .
$$

Then $\gamma_{j}(B)=0$, and $[B, \mathfrak{l}]=f_{j} \bmod \left(V_{j}\right)$, thus $B \in L_{j^{\prime}}(\mathfrak{l}) \cap C_{j} \sim L_{j}(\mathfrak{l})$ which means that $\tau_{j^{\prime \prime}}(\mathfrak{l}) \neq 3$, a contradiction. This proves the first statement of the lemma.

Let $\mathfrak{l}^{\prime} \in G \mathfrak{l}$ such that $\pi_{j-1}(\mathfrak{l})=\pi_{j-1}\left(\mathfrak{l}^{\prime}\right)$, and let $t=\mathfrak{l}_{j}^{\prime}-\mathfrak{l}_{j}$ so that $\mathfrak{l}^{\prime}=$ $\mathfrak{l}+t f_{j} \bmod \left(V_{j}\right)$. Now

$z_{j}\left(\mathfrak{l}^{\prime}\right) f_{j}=\left[r_{j}\left(\mathfrak{l}^{\prime}\right), \mathfrak{l}^{\prime}\right]=\left[r_{j}\left(\mathfrak{l}^{\prime}\right), \mathfrak{l}\right]+t \cdot\left[r_{j}\left(\mathfrak{l}^{\prime}\right), f_{j}\right]=\left[r_{j}\left(\mathfrak{l}^{\prime}\right), \mathfrak{l}\right]+t \cdot \gamma_{j}\left(r_{j}\left(\mathfrak{l}^{\prime}\right)\right) f_{j} \bmod \left(V_{j}\right)$ and hence $[B, \mathfrak{l}]=f_{j} \bmod \left(V_{j}\right)$ where $B=r_{j}\left(\mathfrak{l}^{\prime}\right) /\left(z_{j}\left(\mathfrak{l}^{\prime}\right)-t \gamma_{j}\left(r_{j}\left(\mathfrak{l}^{\prime}\right)\right)\right)$. Thus by the first statement of this lemma we have

$$
q_{j}(\mathfrak{l})=\gamma_{j}\left(r_{j}\left(\mathfrak{l}^{\prime}\right)\right) /\left(z_{j}\left(\mathfrak{l}^{\prime}\right)-t \gamma_{j}\left(r_{j}\left(\mathfrak{l}^{\prime}\right)\right)\right)
$$

and so $-q_{j}(\mathfrak{l})^{-1}=t-q_{j}\left(\mathfrak{l}^{\prime}\right)^{-1}$, which gives the result. Q.E.D.

For each $j, 1 \leq j \leq n$, let $\mu_{j}$ denote the character of $G$ defined by $\mu_{j}(s)=\exp \left(\gamma_{j}(\log (s))\right), s \in G$.

Corollary 2.2. Let $j \in \varphi$. Then $q_{j}$ is $G$-semi-invariant on $\Omega$ with multiplier $\mu_{j}^{-1}$.

Proof. Let $s \in G$, and let $A=\mu_{j}(s)^{-1} z_{j}(\mathfrak{l})^{-1} \operatorname{Ad}(s) r_{j}(\mathfrak{l})$. Then $[A, s \mathfrak{l}]=$ $f_{j} \bmod \left(V_{j}\right)$, and hence

$$
q_{j}(s \mathfrak{l})=\gamma_{j}(A)=\mu_{j}(s)^{-1} z_{j}(\mathfrak{l})^{-1} \gamma_{j}\left(\operatorname{Ad}(s) r_{j}(\mathfrak{l})\right)=\mu_{j}(s)^{-1} q_{j}(\mathfrak{l}) . \quad \text { Q.E.D. }
$$


Let $\mathfrak{l} \in \Omega$ and let $j \in \varphi$. Let $U$ be the irreducible submodule of $W_{j^{\prime \prime}}$ canonically isomorphic to $V_{j^{\prime}} / V_{j^{\prime \prime}}$. The following lemma clarifies the $G$-quotient space structure of $\pi_{j^{\prime \prime}}(\mathfrak{l})+U$ and in particular identifies the unique "singular" point in $\pi_{j^{\prime \prime}}(\mathfrak{l})+U$. Now if $\Omega$ is the minimal layer, then the generic points in $\pi_{j^{\prime \prime}}(\mathfrak{l})+U$ must be contained in $\pi_{j^{\prime \prime}}(\Omega)$. We do not know if this is the case for the singular layers. However, the following shows that this is always the case if $\operatorname{dim}(U)=2$.

Lemma 2.3. Fix $\mathfrak{l} \in \Omega$, let $j \in J$ such that $\tau_{j}=3$, and let $U$ be defined as above. Then

$$
\left\{\mathfrak{l}^{\prime} \in \pi_{j^{\prime \prime}}(\mathfrak{l})+U: \tau_{j}(\mathfrak{l})=3\right\}=\pi_{j^{\prime \prime}}(\mathfrak{l})+U \sim\left\{\mathfrak{l}_{0}\right\},
$$

where $\mathfrak{l}_{0} \in\left(\pi_{j^{\prime \prime}}(\mathfrak{l})+U\right)$ is defined by $\mathfrak{l}_{0}\left(Z_{j}\right)=\mathfrak{l}_{j}-q_{j}(\mathfrak{l})^{-1}$. If $j \notin I$, then

$$
\left(\pi_{j^{\prime \prime}}(\mathfrak{l})+U\right) \cap \pi_{j^{\prime \prime}}(\Omega)=\left(\pi_{j^{\prime \prime}}(\mathfrak{l})+U\right) \sim\left\{\mathfrak{l}_{0}\right\} .
$$

Proof. Since $\tau_{j}=3, j=i_{k} \in i(\mathfrak{l})$, for some $k(1 \leq k \leq d / 2)$. For $r<k$, the condition that $i_{r}\left(\mathfrak{l}^{\prime}\right)=i_{r}$ or that $j\left(i_{r}\right)\left(\mathfrak{l}^{\prime}\right)=j\left(i_{r}\right)$ is a polynomial condition that depends only on $\pi_{i_{r}}\left(\mathfrak{l}^{\prime}\right)$. Hence if $\mathfrak{l}^{\prime} \in \pi_{j^{\prime \prime}}(\mathfrak{l})+U$, then $i_{r}\left(\mathfrak{l}^{\prime}\right)=i_{r}$ and $j\left(i_{r}\right)\left(\mathfrak{l}^{\prime}\right)=j\left(i_{r}\right), 1 \leq r \leq k-1$. Note also that in this case $\mathfrak{h}_{r}\left(\mathfrak{l}^{\prime}\right)=\mathfrak{h}_{r}(\mathfrak{l})$; we denote $\mathfrak{h}_{r}(\mathfrak{l})=\mathfrak{h}_{r}, 1 \leq r \leq k-1$. We claim that for any $\mathfrak{l}^{\prime} \in \pi_{j^{\prime \prime}}(\mathfrak{l})+U$, if $j \in J_{\mathfrak{l}^{\prime}}$, then $j=i_{k}\left(\mathfrak{l}^{\prime}\right)$ and $j\left(i_{k}\right)=j\left(i_{k}\right)\left(\mathfrak{l}^{\prime}\right)$. Let $\mathfrak{l}^{\prime} \in \pi_{j^{\prime \prime}}(\mathfrak{l})+U$ such that $j \in J_{l^{\prime}}$. From the properties of the sequences $i(\mathfrak{l}), j(\mathfrak{l})$, we must have $j=i_{k}\left(\mathfrak{l}^{\prime}\right)$. Now let $j^{*}$ be the smallest $j$ such that for some $\mathfrak{l}^{\prime} \in \pi_{j^{\prime \prime}}(\mathfrak{l})+U$, $\mathfrak{s}_{j} \cap \mathfrak{h}_{k-1} \not \subset \mathfrak{h}_{k}\left(\mathfrak{l}^{\prime}\right)$. We can fix $Z \in \mathfrak{s}_{j^{*}} \cap \mathfrak{h}_{k-1} \sim \mathfrak{s}_{j^{*}-1}$ and $W \in \mathfrak{h}_{k-1} \cap \mathfrak{s}_{j}$ such that for $\mathfrak{l}^{\prime} \in \pi_{j^{\prime \prime}}(\mathfrak{l})+U, j\left(i_{k}\right)\left(\mathfrak{l}^{\prime}\right)=j^{*}$ if and only if $\mathfrak{l}^{\prime}([Z, W]) \neq 0$. Now there is a constant $c$ such that for all $\mathfrak{l}^{\prime} \in \pi_{j^{\prime \prime}}(\mathfrak{l})+U, \mathfrak{l}^{\prime}([Z, W])=\gamma_{j}(Z) \mathfrak{l}^{\prime}\left(Z_{j}\right)+c$. Hence the set $D=\left(\pi_{j^{\prime \prime}}(\mathfrak{l})+U\right) \cap\left\{\mathfrak{l}^{\prime}: j\left(i_{k}\right)\left(\mathfrak{l}^{\prime}\right)=j^{*}\right\}$ consists of all but a single point of $\pi_{j^{\prime \prime}}(\mathfrak{l})+U$. But the set $G \mathfrak{l} \cap\left(\pi_{j^{\prime \prime}}(\mathfrak{l})+U\right)$ consists of more than a single point, and therefore $\mathfrak{l} \in D, j^{*}=j\left(i_{k}\right)$, and

$$
D=\left\{\mathfrak{l}^{\prime} \in \pi_{j^{\prime \prime}}(\mathfrak{l})+U: j\left(i_{k}\right)\left(\mathfrak{l}^{\prime}\right)=j\left(i_{k}\right)\right\} .
$$

It follows from Lemma 1.7 that for every $\mathfrak{l}^{\prime} \in D, \tau_{j}\left(\mathfrak{l}^{\prime}\right)=3$. To see that $\mathfrak{l}_{0} \notin D$, note that for $\mathfrak{l}^{\prime} \in D, q_{j}\left(\mathfrak{l}^{\prime}\right) \neq 0$, and

$$
\mathfrak{l}^{\prime}\left(Z_{j}\right)=\mathfrak{l}_{0}\left(Z_{j}\right)+q_{j}\left(\mathfrak{l}^{\prime}\right)^{-1} .
$$

Hence $D=\left\{\mathfrak{l}^{\prime} \in \pi_{j^{\prime \prime}}(\mathfrak{l})+U: \mathfrak{l}^{\prime} \neq \mathfrak{l}_{0}\right\}$.

Suppose that $j \notin I$. Set $S=\left(\pi_{j^{\prime \prime}}(\mathfrak{l})+U\right) \cap \pi_{j^{\prime \prime}}(\Omega) ; S \subset D$. Suppose that $S \neq D$. Choose $\mathfrak{l}^{\prime} \in D \sim S$, and set $T\left(\mathfrak{l}^{\prime}\right)=\left(\pi_{j^{\prime \prime}}\left(\mathfrak{l}^{\prime}\right)+U\right) \cap G \mathfrak{l}^{\prime}$. Then $T\left(\mathfrak{l}^{\prime}\right) \subset D \sim S$. If $A$ is a one dimensional affine subset of $\pi_{j^{\prime \prime}}(\mathfrak{l})+U$, then for each $\mathfrak{l}^{\prime} \in D, A$ meets $T\left(\mathfrak{l}^{\prime}\right)$ in a countably infinite discrete set of points. Hence $A \cap S$ has infinitely many components. But $A \cap S$ is a semialgebraic subset of $A$; in fact, it is the image of the algebraic set $\pi_{j^{\prime \prime}}^{-1}(A) \cap \Omega$ under the projection $\pi_{j^{\prime \prime}}$. Hence $A \cap S$ can have only finitely many components. Therefore $D=S$ and the lemma is proved. Q.E.D.

We now focus attention on the case $j \in l$. Let $F$ be the cover of $\Omega$ defined in Lemma 1.3, and let $O$ and $O^{\prime}$ be two elements of $F$. Let $z_{j}: O \rightarrow S^{1}$, $z_{j}^{\prime}: O^{\prime} \rightarrow S^{1}$ as in Proposition 1.5. If $\mathfrak{l} \in O \cap O^{\prime}$, then $z_{j}(\mathfrak{l})= \pm z_{j}^{\prime}(\mathfrak{l})$. Let $P S^{1}=S^{1} /\{1,-1\}$ and let $p: S^{1} \rightarrow P S^{1}$ be the quotient mapping. Then 
$\zeta_{j}=p \circ z_{j}$ is a well-defined mapping from $\Omega$ into $P S^{1}$. For each $j(1 \leq j \leq n)$ set $\nu_{j}(s)=\mu_{j}(s) /\left|\mu_{j}(s)\right|, s \in G$. We shall need the fact that if $\tau_{j}=2$, then $\zeta_{j}$ is $G$-semi-invariant with multiplier $\nu_{j}$.

Lemma 2.4. Let $j \in J$ such that $\operatorname{dim}\left(L_{j^{\prime}}(\mathfrak{l}) / L_{j^{\prime \prime}}(\mathfrak{l})\right)=1$. Let $\mathfrak{l} \in \Omega$ and let $s \in G$. Then $\zeta_{j}(s \mathfrak{l})=\nu_{j}(s) \zeta_{j}(\mathfrak{l})$.

Proof. Let $s \in G, \mathfrak{l} \in \Omega$. Fix $O, O^{\prime} \in F$ such that $\mathfrak{l} \in O, s \mathfrak{l} \in O^{\prime}$, and let $z_{j}(\mathfrak{l}), z_{j}(s \mathfrak{l})$ as in Proposition 1.5. Then $\operatorname{Ad}(s) r_{j}(\mathfrak{l}) \in L_{j^{\prime}}(s \mathfrak{l}) \sim L_{j^{\prime \prime}}(s \mathfrak{l})$. Since $\operatorname{dim}_{\mathbf{R}}\left(L_{j^{\prime}}(s \mathfrak{l}) \cap \mathfrak{g} / L_{j^{\prime \prime}}(s \mathfrak{l}) \cap \mathfrak{g}\right)=1$, there is a (unique) real number $t=t(s, \mathfrak{l})$ such that $r_{j}(s \mathfrak{l})-t(s, \mathfrak{l}) \operatorname{Ad}(s) r_{j}(\mathfrak{l}) \in L_{j^{\prime \prime}}(\mathfrak{l})$. Let $Z=r_{j}(s \mathfrak{l})-t(s, \mathfrak{l}) \operatorname{Ad}(s) r_{j}(\mathfrak{l})$. Now

$$
\left(z_{j}(s \mathfrak{l})-t(s, \mathfrak{l}) \mu_{j}(s) z_{j}(\mathfrak{l})\right) f_{j}=[Z, \mathfrak{l}]=0 \bmod \left(V_{j^{\prime \prime}}\right) .
$$

Hence for each $s \in G, \mathfrak{l} \in \Omega$, we have $t(s, \mathfrak{l}) \mu_{j}(s)=z_{j}(s \mathfrak{l}) / z_{j}(\mathfrak{l})$. Therefore $t(s, \mathfrak{l}) \mu_{j}(s)= \pm \nu_{j}(s)$, which obtains the result. Q.E.D.

Lemma 2.5. Let $j \in \mathfrak{l}$. Then for any $\mathfrak{l} \in \Omega, L_{j^{\prime}}(\mathfrak{l}) \subset C_{j}$.

Proof. Let $A \in L_{j^{\prime}}(\mathfrak{l})$. First suppose that $j=i_{k}$ (some $\left.k, 1 \leq k \leq d / 2\right)$. There is $Y \in \mathfrak{s}_{j^{\prime \prime}} \cap R\left(\mathfrak{l}, \mathfrak{h}_{k-1}(\mathfrak{l})\right) \sim \mathfrak{s}_{j^{\prime}}$. Since $L_{j^{\prime}}(\mathfrak{l}) \subset \mathfrak{h}_{k-1}(\mathfrak{l})$ we have $\left[A, r_{j}(\mathfrak{l})\right]$ $\in \mathfrak{h}_{k-1}(\mathfrak{l})$ and $[A, Y] \in \mathfrak{h}_{k-1}(\mathfrak{l})$. By Proposition 1.5, $r_{j}(\mathfrak{l}) \in C_{j}$, and hence

$$
O=\mathfrak{l}\left(\left[A,\left[r_{j}(\mathfrak{l}), Y\right]\right]\right)=\mathfrak{l}\left(\left[r_{j}(\mathfrak{l}),[A, Y]\right]\right) .
$$

But if $\gamma_{j}(A) \neq 0$, then $\gamma_{j}(A) \notin \mathbf{R}$, and hence $Y$ and $[A, Y]$ span $\mathfrak{s}_{j^{\prime \prime}} \cap \mathfrak{h}_{k-1}(\mathfrak{l})$ modulo $\mathfrak{s}_{j^{\prime}} \cap \mathfrak{h}_{k-1}(\mathfrak{l})$. This contradicts the definition of $r_{j}(\mathfrak{l})$.

Suppose that $j=j\left(i_{k}\right)$. Here we have $X \in \mathfrak{s}_{j^{\prime \prime}} \cap \mathfrak{h}_{k}(\mathfrak{l}) \sim \mathfrak{s}_{j^{\prime}}$, and $\left[A, r_{j}(\mathfrak{l})\right] \in$ $\mathfrak{s}_{i_{k}^{\prime \prime}}$. Also $[A, X] \in \mathfrak{s}_{j^{\prime \prime}} \cap \mathfrak{h}_{k-1}(\mathfrak{l})$. Now a similar application of the Jacobi relation shows that $A \in C_{j}$. Q.E.D.

Denote by $J^{\prime}$ the set of all $j \in J$ such that $j \notin I^{\prime \prime}$; write $J^{\prime}=\left\{j_{1}<\right.$ $\left.j_{2}<\cdots<j_{h}\right\}$. Define a subset $R(\Omega)$ of $\mathbf{C}^{h}$ as follows. Fix $j=j_{m} \in J^{\prime}$; first suppose that $j \in I^{\prime}$. If $j \notin \varphi$, set $R(\Omega)_{m}=\mathbf{R}$, while if $j \in \varphi$ set $R(\Omega)_{m}=\mathbf{R}^{*}$. Now suppose that $j \notin I$. If $j \notin \varphi$ set $R(\Omega)_{m}=\mathbf{C}$, if $j \in \varphi$ set $R(\Omega)_{m}=\mathbf{C}^{*}$. Set

$$
R(\Omega)=R(\Omega)_{1} \times R(\Omega)_{2} \times \cdots \times R(\Omega)_{h} .
$$

For $j=j_{m} \in \varphi$, consider the action of $G$ on $R(\Omega)_{m}$ defined by $s \cdot r=\mu_{j}(s) r$; clearly $R(\Omega)_{m} / G \cong S^{0}$ or $S^{1}$ according as $j \in I^{\prime}$ or $j \notin I^{\prime}$, respectively. Define an equivalence relation $\approx$ on $R(\Omega)$ by $r \approx r^{\prime}$ if and only if for each $m$ such that $j_{m} \in \varphi, G r_{m}=G r_{m}^{\prime}$. Let

$$
T(\Omega)=R(\Omega) / \approx .
$$

Note that $T(\Omega)$ is homeomorphic to a product $\prod S^{i}$, where $i=0$ or 1 .

Let $T \in T(\Omega)$. Suppose that for each $j=j \in l$, we have a smooth function $\chi_{j}=\chi_{j}\left(z_{1}, z_{2}, \ldots, z_{j-1}\right)$ into $P S^{1}$. For each $\left(z_{1}, z_{2}, \ldots, z_{j-1}\right)$, let

$$
R_{j}\left(z_{1}, z_{2}, \ldots, z_{j-1}\right) \subset \mathbf{C}
$$

be the R-subspace of $\mathbf{C}$ "through" $\chi_{j}\left(z_{1}, z_{2}, \ldots, z_{j-1}\right)$. Let

$$
T_{\chi}=\left\{\left(z_{1}, z_{2}, \ldots, z_{h}\right) \in T: \text { for each } j \in \imath, z_{j} \in R_{j}\left(z_{1}, z_{2}, \ldots, z_{j-1}\right)\right\} .
$$

Then $T_{\chi}$ is a submanifold of $T$. 
Proposition 2.6. There is a G-invariant partition of $\mathfrak{g}^{*}$ into algebraic sets $\Omega$ such that for each such set $\Omega$, there are $n$ analytic functions $\left\{P_{j}\right\}$ in $h+n$ complex variables $z_{1}, z_{2}, \ldots, z_{h}, \mathfrak{l}_{1}, \mathfrak{l}_{2}, \ldots, \mathfrak{l}_{n}$ and indices $J=\left\{j_{k}\right\}, l \subset J$, $\varphi \subset J$ with $1 \leq j_{1}<j_{2}<\cdots<j_{d} \leq n$, having the following properties.

(1) For each $\mathfrak{l} \in \Omega$, let $T(\mathfrak{l})$ be the unique element of $T(\Omega)$ such that for each $j=j_{m} \in \varphi, T(\mathfrak{l})_{m}=\left\{q_{j}\left(\mathfrak{l}^{\prime}\right)^{-1}: \mathfrak{l}^{\prime} \in G \mathfrak{l}\right\}$. For each $j \in \mathfrak{l}$, there is a function $\chi_{j}\left(z_{1}, z_{2}, \ldots, z_{j-1}, \mathfrak{l}\right)$ into $P S^{1}$ such that the mapping $\left(z_{1}, z_{2}, \ldots, z_{h}\right) \rightarrow$ $\sum_{j} P_{j}\left(z_{1}, z_{2}, \ldots, z_{h}, \mathfrak{l}\right) f_{j}$ is a diffeomorphism of $T(\mathfrak{l})_{\chi}$ with the coadjoint orbit of $\mathfrak{l}$. If $\mathfrak{l}^{\prime} \in G \mathfrak{l}$ and $T\left(\mathfrak{l}^{\prime}\right)_{\chi^{\prime}}$ is as above, then $T\left(\mathfrak{l}^{\prime}\right)_{\chi^{\prime}}=T(\mathfrak{l})_{\chi}$.

(2) Let $j_{m} \in J^{\prime}$. Then $P_{j}(z, \mathfrak{l})=z_{m}\left(z_{1}, z_{2}, \ldots, z_{m-1}\right)$. If $\tau_{j}=1$, then $P_{j}(z, \mathfrak{l})=z_{m}$.

(3) For each $j, P_{j}(z, \mathfrak{l})$ depends only on those $z_{m}$ for which $j_{m} \leq j$.

(4) Let $\mathfrak{l} \in \Omega$ and let $z \in T(\mathfrak{l})_{\chi}$. Then for each $j, P_{j}(z, \mathfrak{l})=P_{j}(z, s \mathfrak{l})$.

Proof. Let $\wp$ be the layering of $\mathfrak{g}^{*}$ define above; each $\Omega \in \wp$ is an algebraic subset of $\mathfrak{g}^{*}$ (defined by explicit polynomials).

Now let $J \in \mathfrak{j}, J \neq \varnothing$, and write $J=\left\{j_{1}<j_{2}<\cdots<j_{d}\right\}$. Let $\alpha \in A_{J}$ and $\varphi \in \vartheta_{\alpha}$ and fix $\Omega=\Omega_{\alpha, \varphi}$. Define $l \subset J$ as above. Let $F$ be the covering of $\Omega_{\alpha}$ as in Lemma 1.3. Fix $\mathfrak{l} \in \Omega$ and let $O \in F$ such that $\mathfrak{l} \in O$. Set $g_{k}(x, \mathfrak{l})=\exp \left(x r_{j_{k}}(\mathfrak{l})\right), x \in \mathbf{R}, 1 \leq k \leq d$, and set

$$
g(t, \mathfrak{l})=g_{1}\left(t_{1}, \mathfrak{l}\right) g_{2}\left(t_{2}, \mathfrak{l}\right) \cdots g_{d}\left(t_{d}, \mathfrak{l}\right), \quad t \in \mathbf{R}^{d} .
$$

For each $j \in J$, let $b_{j}(\mathfrak{l})=\gamma_{j}\left(r_{j}(\mathfrak{l})\right), \mathfrak{l} \in O$; for each $j, b_{j}$ is an analytic function on $O$. The proof of Proposition 5 in [17] shows that for each $\mathfrak{l} \in O$, $G \mathfrak{l}=\left\{g(t, \mathfrak{l}) \mathfrak{l}: t \in \mathbf{R}^{d}\right\}$, and $g(t, \mathfrak{l}) \mathfrak{l}=\sum Q_{j}(t, \mathfrak{l}) f_{j}$ where the $Q_{j}$ satisfy the following:

(1) $Q_{j}(t, \mathfrak{l})=0\left(t_{1}, t_{2}, \ldots, t_{k}\right)$ where $k$ is such that $j_{k} \leq j<j_{k+1}$,

(2) if $j=j_{k} \in J$ and $\operatorname{dim}\left(L_{j^{\prime}}(\mathfrak{l}) / L_{j^{\prime \prime}}(\mathfrak{l})\right)=1$, then

$$
Q_{j}(t, \mathfrak{l})=t_{k} F\left(b_{j}(\mathfrak{l}) t_{k}\right) \exp \left(L_{j}(t, \mathfrak{l})\right) z_{j}(\mathfrak{l})+S_{j}(t, \mathfrak{l})
$$

where $F$ denotes the analytic function defined by $F(z)=\left(e^{z}-1\right) / z$, and

(3) if $j=j_{k} \in J^{\prime}$ and $\operatorname{dim}\left(L_{j^{\prime}}(\mathfrak{l}) / L_{j^{\prime \prime}}(\mathfrak{l})\right)=2$, then

$$
Q_{j}(t, \mathfrak{l})=\left(z_{j}(\mathfrak{l}) t_{k}+z_{j+1}(\mathfrak{l}) t_{k+1}\right) \exp \left(L_{j}(t, \mathfrak{l})\right)+S_{j}(t, \mathfrak{l}) .
$$

In each case (2) and (3), $L_{j_{k}}(t, \mathfrak{l})$ is a linear form in $t_{1}, t_{2}, \ldots, t_{k-1}\left(L_{j_{1}}(t, \mathfrak{l})\right.$ $\equiv 0)$; in fact we have

$$
\exp \left(L_{j}(t, \mathfrak{l})\right)=\mu_{j}\left(g_{1}\left(t_{1}, \mathfrak{l}\right) g_{2}\left(t_{2}, \mathfrak{l}\right) \cdots g_{k-1}\left(t_{k-1}, \mathfrak{l}\right)\right) .
$$

Thus by construction of $r_{j_{r}}(\mathfrak{l}) \quad(1 \leq r \leq k-1), L_{j}(t, \mathfrak{l})$ depends only on $\pi_{j-1}(\mathfrak{l})$. For each $\mathfrak{l}$, the function $S_{j_{k}}(t, \mathfrak{l})$ depends only on $t_{1}, t_{2}, \ldots, t_{k-1}$, and for each $j, S_{j}(t, \mathfrak{l})$ is of the form

$$
S_{j}(t, \mathfrak{l})=\exp \left(L_{j}(t, \mathfrak{l})\right) \mathfrak{l}_{j}+Y_{j}(t, \mathfrak{l}),
$$

where

$$
Y_{j}(t, \mathfrak{l})=\pi_{j^{\prime}}(\mathfrak{l})\left(\operatorname{Ad}\left(g_{1}\left(t_{1}, \mathfrak{l}\right) g_{2}\left(t_{2}, \mathfrak{l}\right) \cdots g_{k-1}\left(t_{k-1}, \mathfrak{l}\right)\right)^{-1} Z_{j}\right) \quad\left(Y_{j_{1}}(t, \mathfrak{l}) \equiv 0\right) .
$$

Note that if $j \in I^{\prime}$, then $Q_{j}$ is real, and recall that (by Proposition 1.5) if $\tau_{j}=1$ or 2 , then $\gamma_{j}\left(r_{j}(\mathfrak{l})\right)=0$. Write $J^{\prime}=\left\{j_{1}<j_{2}<\cdots<j_{h}\right\}$ as before. Let 
$j=j_{m} \in J^{\prime}$; we define $z_{m}$ as follows. If $\tau_{j}=1$, set $z_{m}=Q_{j}(t, \mathfrak{l})$. If $\tau_{j}=2$, then

$$
Q_{j}(t, \mathfrak{l})=\left(t_{k} z_{j}(\mathfrak{l})+\mathfrak{l}_{j}\right) \exp \left(L_{j}(t, \mathfrak{l})\right)+Y_{j}(t, \mathfrak{l}) \quad(\text { some } k, \quad 1 \leq k \leq d) .
$$

We set

$$
\begin{aligned}
c_{j}(t, \mathfrak{l}) & =\nu_{j}\left(g_{1}\left(t_{1}, \mathfrak{l}\right) g_{2}\left(t_{2}, \mathfrak{l}\right) \cdots g_{k-1}\left(t_{k-1}, \mathfrak{l}\right)\right) z_{j}(\mathfrak{l}) \\
& =\exp \left(L_{j}(t, \mathfrak{l})\right) z_{j}(\mathfrak{l}) /\left|\exp \left(L_{j}(t, \mathfrak{l})\right)\right|
\end{aligned}
$$

and

$$
z_{m}=c_{j}(t, \mathfrak{l}) \operatorname{Re}\left[c_{j}(t, \mathfrak{l})^{-1} Q_{j}(t, \mathfrak{l})\right]
$$

Note that

$$
c_{j}(t, \mathfrak{l})^{-1} Q_{j}(t, \mathfrak{l})=t_{k}\left|\exp \left(L_{j}(t, \mathfrak{l})\right)\right|+c_{j}(t, \mathfrak{l})^{-1}\left[\mathfrak{l}_{j} \exp \left(L_{j}(t, \mathfrak{l})\right)+Y_{j}(t, \mathfrak{l})\right]
$$

Thus $c_{j}(t, \mathfrak{l}) i \operatorname{Im}\left(c_{j}(t, \mathfrak{l})^{-1} Q_{j}(t, \mathfrak{l})\right)$ depends only on $t_{1}, t_{2}, \ldots, t_{k-1}$. Note also that by Lemma 2.3, $p\left(c_{j}(t, \mathfrak{l})\right)=\zeta_{j}\left(g_{1}\left(t_{1}, \mathfrak{l}\right) g_{2}\left(t_{2}, \mathfrak{l}\right) \cdots g_{k-1}\left(t_{k-1}, \mathfrak{l}\right) \mathfrak{l}\right)$.

Finally if $\tau_{j}=3$, set

$$
z_{m}=\exp \left(b_{j}(\mathfrak{l}) t_{k}\right) \exp \left(L_{j}(t, \mathfrak{l})\right) z_{j}(\mathfrak{l}) / b_{j}(\mathfrak{l}) .
$$

Note that in this latter case, $z_{m}=q_{j}(g(t, \mathfrak{l}) \mathfrak{l})^{-1}$. In any case, the properties of the functions $Q_{j}$ above (and that fact that $G$ is exponential) allow us to solve for each $t_{k}$ in terms of $\mathfrak{l}, z_{m}$ and the variables $t_{1}, t_{2}, \ldots, t_{k-1}$. Thus we can solve for $t_{k}$ in terms of $\mathfrak{l}$ and the complex variables $z_{1}, z_{2}, \ldots, z_{m}$ where $j_{m} \leq j_{k}<j_{m+1}$. We write $t_{k}=\Phi_{k}(z, \mathfrak{l})$ and substitute into $Q_{1}$ to obtain $P_{1}$, $Q_{2}$ to obtain $P_{2}$, and so on. Thus

$$
P_{j}(z, \mathfrak{l})=Q_{j}\left(\Phi_{1}\left(z_{1}, \mathfrak{l}\right), \Phi_{2}\left(z_{1}, z_{2}, \mathfrak{l}\right), \ldots, \Phi_{k}\left(z_{1}, z_{2}, \ldots, z_{m}, \mathfrak{l}\right), \mathfrak{l}\right)
$$

where $k(1 \leq k \leq d)$ is such that $j_{k} \leq j<j_{k}+1$ and $m(1 \leq m \leq h)$ is such that $j_{m} \leq j_{k}<j_{m}+1$. Note that for each $z, \varphi_{j}(z, \mathfrak{l})$ depends only on $\pi_{j}(\mathfrak{l})$. For $j \in l$, set

$$
\begin{aligned}
& \quad \chi_{j}\left(z_{1}, z_{2}, \ldots, z_{m-1}, \mathfrak{l}\right) \\
& \quad=p\left(c_{j}\left(\Phi_{1}\left(z_{1}, \mathfrak{l}\right), \Phi_{2}\left(z_{1}, z_{2}, \mathfrak{l}\right), \ldots, \Phi_{k-1}\left(z_{1}, z_{2}, \ldots, z_{m-1}, \mathfrak{l}\right), \mathfrak{l}\right)\right) .
\end{aligned}
$$

For each fixed $\left(z_{1}, z_{2}, \ldots, z_{m-1}\right), z_{m}$ runs through $R_{j}\left(z_{1}, z_{2}, \ldots, z_{m-1}, \mathfrak{l}\right)$ the R-subspace corresponding to $\chi_{j}\left(z_{1}, z_{2}, \ldots, z_{m-1}, \mathfrak{l}\right)$.

In this way, we obtain analytic functions $P_{1}, P_{2}, \ldots, P_{n}$ satisfying conditions (2) and (3) above. Moreover, for each $\mathfrak{l} \in O$, the mapping $\mathfrak{l} \rightarrow$ $\sum P_{j}(z, \mathfrak{l}) f_{j}$ is a diffeomorphism of $T(\mathfrak{l})_{\chi}$ with the orbit of $\mathfrak{l}$, and by Lemma 2.4 , the set $T(\mathfrak{l})_{\chi}$ is independent of choice of $O \in F$. We need to show that the set $T(\mathfrak{l})_{\chi}$ depends only on the orbit of $\mathfrak{l}$, that for each $z \in T(\mathfrak{l})_{\chi}$ fixed, $P_{j}(z, \cdot)$ is $G$-invariant $(1 \leq j \leq n)$, and that this construction is independent of the open set $O \in F$ chosen above.

Fix $s \in G$, let $\mathfrak{l} \in \Omega$ and let $O, O^{\prime} \in F$ such that $\mathfrak{l} \in O$ and $s \mathfrak{l} \in O^{\prime}$. Let $\mathfrak{l}^{\prime}=\sum P_{j}(z, \mathfrak{l}) f_{j}$ and $\mathfrak{l}^{\prime \prime}=\sum P_{j}^{\prime}(z, s \mathfrak{l}) f_{j}$ is obtained above. Let $T^{\prime}, T^{\prime \prime} \in \mathbf{R}^{d}$ such that $\mathfrak{l}^{\prime}=g\left(t^{\prime}, \mathfrak{l}\right) \mathfrak{l}, \mathfrak{l}^{\prime \prime}=g^{\prime}\left(t^{\prime \prime}, s \mathfrak{l}\right) s \mathfrak{l}$. Set

$$
g_{0}^{\prime}=g_{1}\left(t_{1}^{\prime}, \mathfrak{l}\right) g_{2}\left(t_{2}^{\prime}, \mathfrak{l}\right) \cdots g_{k-1}\left(t_{k-1}^{\prime}, \mathfrak{l}\right),
$$

$g_{k}^{\prime}=g_{k}\left(t_{k}^{\prime}, \mathfrak{l}\right)$ and define $g_{0}^{\prime \prime}$ and $g_{k}^{\prime \prime}$ similarly, so that $\mathfrak{l}^{\prime}=g_{0}^{\prime} g_{k}^{\prime} \mathfrak{l}$ and $\mathfrak{l}^{\prime \prime}=$ $g_{0}^{\prime \prime} g_{k}^{\prime \prime} s l$. 
We prove that $T(\mathfrak{l})_{\chi}$ depends only on the orbit of $\mathfrak{l}$ by showing that for each $j_{m} \in J^{\prime}$ the set $\left\{\left(z_{1}, z_{2}, \ldots, z_{m}\right):\left(z_{1}, z_{2}, \ldots, z_{h}\right) \in T(\mathfrak{l})_{\chi}\right\}$ depends only on the orbit of $\mathfrak{l}$. As usual this is done by induction. Let $j_{m} \in J^{\prime}$. Suppose that the set $\left\{\left(z_{1}, z_{2}, \ldots, z_{m-1}\right):\left(z_{1}, z_{2}, \ldots, z_{h}\right) \in T(\mathfrak{l})_{\chi}\right\}$ depends only on the orbit of $\mathfrak{l}$. We may assume that $j \in l$. Let $z$ belong to this set; we show that $\chi_{j}(z, \mathfrak{l})=\chi_{j}(z, s \mathfrak{l})$. We have $\chi_{j}(z, \mathfrak{l})=\nu_{j}\left(g_{0}^{\prime}\right) \zeta_{j}(\mathfrak{l})$ and by Lemma 2.4, $\chi_{j}(z, s \mathfrak{l})=\nu_{j}\left(g_{0}^{\prime \prime}\right) \zeta_{j}(s \mathfrak{l})=\nu_{j}\left(g_{0}^{\prime \prime} s\right) \zeta_{j}(\mathfrak{l})$. By induction $\pi_{j-1}\left(\mathfrak{l}^{\prime}\right)=$ $\pi_{j-1}\left(l^{\prime \prime}\right)$, hence $g_{0}^{\prime} \pi_{j-1}(\mathfrak{l})=g_{0}^{\prime \prime} s \pi_{j-1}(\mathfrak{l})$. By Lemma $2.5, \log \left(g_{0}^{\prime-1} g_{0}^{\prime \prime} s\right) \in C_{j}$, and hence $\nu_{j}\left(g_{0}^{\prime}\right)=\nu_{j}\left(g_{0}^{\prime \prime} s\right)$. Hence we have $\chi_{j}(z, \mathfrak{l})=\chi_{j}(z, s \mathfrak{l})$ and the set $\left\{\left(z_{1}, z_{2}, \ldots, z_{m}\right):\left(z_{1}, z_{2}, \ldots, z_{h}\right) \in T(\mathfrak{l})_{\chi}\right\}$ depends only on the orbit of $\mathfrak{l}$.

Now fix $z=\left(z_{1}, z_{2}, \ldots, z_{h}\right) \in T(\mathfrak{l})_{\chi}$. We show that for each $j, \pi_{j^{\prime \prime}}\left(\mathfrak{l}^{\prime}\right)=$ $\pi_{j^{\prime \prime}}\left(\mathfrak{l}^{\prime \prime}\right)$, by induction on $j$. We may assume that $j \notin I^{\prime \prime}$. Suppose that $j=1$; we consider the two cases (1) $1 \notin J,(2) 1 \in J$. For simplicity of notation, set $\pi=\pi_{j^{\prime \prime}}$.

Case (1). In this case $P_{1}(z, \mathfrak{l})=P_{1}(z, s \mathfrak{l})=\mathfrak{l}_{1}$ by construction, hence $\pi\left(\mathfrak{l}^{\prime}\right)=$ $\pi\left(l^{\prime \prime}\right)=\mathfrak{l}_{1}$.

Case (2). Here $\tau_{1} \neq 0$. If $\tau_{1}=1$, then $P_{1}(z, \mathfrak{l})=P_{1}(z, s \mathfrak{l})=z_{1}$, thus $\pi\left(l^{\prime}\right)=\pi\left(l^{\prime \prime}\right)$. Note that $\tau_{1} \neq 2$, since $C_{1}=$ the centralizer of $Z_{1}$. Suppose that $\tau_{1}=3$. By the form of the function $Q_{1}(t, \mathfrak{l})$, we have that for each $\mathfrak{f} \in G \mathfrak{l}$,

$$
P_{1}(z, \mathfrak{f})=z_{1}+\mathfrak{f}_{1}-q_{1}(\mathfrak{f})^{-1} .
$$

We now apply Lemma 2.1 to obtain that $P_{1}(z, \mathfrak{l})=P_{1}(z, s \mathfrak{l})$, hence $\pi\left(\mathfrak{l}^{\prime}\right)=$ $\pi\left(\mathfrak{l}^{\prime \prime}\right)$.

Now suppose that $j>1$ and that $\pi_{j-1}\left(\mathfrak{l}^{\prime}\right)=\pi_{j-1}\left(\mathfrak{l}^{\prime \prime}\right)$. Let $U$ be the irreducible submodule of $W_{j}$ canonically isomorphic with $V_{j^{\prime}} / V_{j^{\prime \prime}}$. We again consider the two cases: (1) $j \notin J,(2) j \in J$.

Case (1). Since $j \notin J$, either $\tau_{j}=0$, or $\tau_{j}=3$. In the first case, since $\pi\left(\mathfrak{l}^{\prime}\right)$ and $\pi\left(l^{\prime \prime}\right)$ lie in the same $G$-orbit in $W_{j}$, and $\pi_{j-1}\left(\mathfrak{l}^{\prime}\right)=\pi_{j-1}\left(\mathrm{l}^{\prime \prime}\right)$, we have

$$
\pi\left(\mathfrak{l}^{\prime}\right)=G \mathfrak{l} \cap\left(\pi_{j-1}\left(\mathfrak{l}^{\prime}\right)+U\right)=G \mathfrak{l} \cap\left(\pi_{j-1}\left(\mathfrak{l}^{\prime \prime}\right)+U\right)=\pi\left(\mathfrak{l}^{\prime \prime}\right) .
$$

On the other hand, If $\tau_{j}=3$, then letting $\mathfrak{l}_{0} \in \pi\left(\mathfrak{l}^{\prime}\right)+U$ be defined as in Lemma 2.3, we have (by Lemma 2.3),

$$
\pi\left(\mathfrak{l}^{\prime}\right)=\mathfrak{l}_{0}=\pi_{j}\left(\mathfrak{l}^{\prime \prime}\right) .
$$

Case (2). First suppose that $\tau_{j}=1$. We have $j=j_{m} \in J^{\prime}$; hence $P_{j}(z, \mathfrak{l})=$ $P_{j}(z, s \mathfrak{l})=z_{m}$ so that $\pi\left(\mathfrak{l}^{\prime}\right)=\pi_{j-1}\left(\mathfrak{l}^{\prime}\right)+2 \operatorname{Re}\left(z_{m} f_{j}\right)=\pi_{j-1}\left(\mathfrak{l}^{\prime \prime}\right)+2 \operatorname{Re}\left(z_{m} f_{j}\right)=$ $\pi\left(\mathfrak{l}^{\prime \prime}\right)$.

Suppose next that $\tau_{j}=2$. Recall that for some $k \quad(1 \leq k \leq d)$,

$$
\chi_{j}(z, \mathfrak{l})=p\left(c_{j}\left(\Phi_{1}\left(z_{1}, \mathfrak{l}\right), \Phi_{2}\left(z_{1}, z_{2}, \mathfrak{l}\right), \ldots, \Phi_{k-1}\left(z_{1}, z_{2}, \ldots, z_{m-1}, \mathfrak{l}\right), \mathfrak{l}\right)\right) .
$$

Since $c_{j}\left(t^{\prime}, \mathfrak{l}\right) i \operatorname{Im}\left(c_{j}\left(t^{\prime}, \mathfrak{l}\right)^{-1} Q_{j}\left(t^{\prime}, \mathfrak{l}\right)\right)$ depends only on $t_{1}, t_{2}, \ldots, t_{k-1}$, we have

$$
P_{j}(z, \mathfrak{l})=z_{m}+S_{j}(z, \mathfrak{l}),
$$

where $S_{j}(z, \mathfrak{l})$ depends only on $z_{1}, z_{2}, \ldots, z_{m-1}$. Similarly,

$$
P_{j}(z, s \mathfrak{l})=z_{m}+S_{j}(z, s \mathfrak{l}) .
$$


As above, we have $c_{j}\left(t^{\prime}, \mathfrak{l}\right)=\nu_{j}\left(g_{0}^{\prime}\right) z_{j}(\mathfrak{l})=\nu_{j}\left(g_{0}^{\prime \prime} s\right) z_{j}(\mathfrak{l})= \pm c_{j}\left(t^{\prime \prime}, s \mathfrak{l}\right)$, and by Lemma 2.4. $\nu_{j}\left(g_{0}^{\prime}\right) z_{j}(\mathfrak{l})= \pm z_{j}\left(g_{0}^{\prime} \mathfrak{l}\right)$. Set $c=z_{j}\left(g_{0}^{\prime} \mathfrak{l}\right)$. Now

$$
\begin{aligned}
S_{j}(z, \mathfrak{l}) & =c_{j}\left(t^{\prime}, \mathfrak{l}\right) i \operatorname{Im}\left(c_{j}\left(t^{\prime}, \mathfrak{l}\right)^{-1}\left[\mathfrak{l}_{j} \exp \left(L_{j}\left(t^{\prime}, \mathfrak{l}\right)\right)+Y_{j}\left(t^{\prime}, \mathfrak{l}\right)\right]\right) \\
& = \pm c i \operatorname{Im}\left( \pm c^{-1}\left[g_{0}^{\prime} \mathfrak{l}\left(Z_{j}\right)\right]\right)=c i g_{0}^{\prime} \mathfrak{l}\left(\operatorname{Im}\left(c^{-1} Z_{j}\right)\right) .
\end{aligned}
$$

Similarly,

$$
S_{j}(z, s \mathfrak{l})=c i g_{0}^{\prime \prime} s \mathfrak{l}\left(\operatorname{Im}\left(c^{-1} Z_{j}\right)\right) .
$$

Since $\pi_{j-1}\left(g_{0}^{\prime} \mathfrak{l}\right)=\pi_{j-1}\left(g_{0}^{\prime \prime} s \mathfrak{l}\right)$, then for some $x \in \mathbf{R}$,

$$
\pi\left(\exp \left[x r_{j}\left(g_{0}^{\prime} \mathfrak{l}\right)\right] g_{0}^{\prime} \mathfrak{l}\right)=\pi\left(g_{0}^{\prime \prime} s \mathfrak{l}\right) .
$$

But $c=z_{j}\left(g_{0}^{\prime} \mathfrak{l}\right)=\left[\operatorname{ad}^{*}\left(r_{j}\left(g_{0}^{\prime} \mathfrak{l}\right)\right) g_{0}^{\prime} \mathfrak{l}\right]\left(Z_{j}\right)$, hence

$$
\begin{aligned}
& \quad\left[\operatorname{ad}^{*}\left(r_{j}\left(g_{0}^{\prime} \mathfrak{l}\right)\right) g_{0}^{\prime} \mathfrak{l}\right]\left(\operatorname{Im}\left(c^{-1} Z_{j}\right)\right) \\
& \quad=\operatorname{Im}\left\{\left[\operatorname{ad}^{*}\left(r_{j}\left(g_{0}^{\prime} \mathfrak{l}\right)\right) g_{0}^{\prime} \mathfrak{l}\right]\left(Z_{j}\right) /\left[\operatorname{ad}^{*}\left(r_{j}\left(g_{0}^{\prime} \mathfrak{l}\right)\right) g_{0}^{\prime} \mathfrak{l}\right]\left(Z_{j}\right)\right\}=0 .
\end{aligned}
$$

Since $r_{j}\left(g_{0}^{\prime} \mathfrak{l}\right) \in L_{j^{\prime}}(\mathfrak{l})^{\prime}$ and $\gamma_{j}\left(r_{j}\left(g_{0}^{\prime} \mathfrak{l}\right)\right)=0$, then for each $n=1,2, \ldots$,

$$
\left[\left(\operatorname{ad}^{*}\right)^{n} r_{j}\left(g_{0}^{\prime} \mathfrak{l}\right) g_{0}^{\prime} \mathfrak{l}\right]\left(\operatorname{Im}\left(c^{-1} Z_{j}\right)\right)=0
$$

Hence,

$$
g_{0}^{\prime \prime} s \mathfrak{l}\left(\operatorname{Im}\left(c^{-1} Z_{j}\right)\right)=\left[\exp \left(x r_{j}\left(g_{0}^{\prime} \mathfrak{l}\right)\right) g_{0}^{\prime} \mathfrak{l}\right]\left(\operatorname{Im}\left(c^{-1} Z_{j}\right)\right)=g_{0}^{\prime} \mathfrak{l}\left(\operatorname{Im}\left(c^{-1} Z_{j}\right)\right) .
$$

Thus $R_{j}(z, s \mathfrak{l})=R_{j}(z, \mathfrak{l})$ and $P_{j}(z, s \mathfrak{l})=P_{j}(z, \mathfrak{l})$.

Finally, suppose that $\tau_{j}=3$. Then $j=j_{m} \in J^{\prime}$. Recall that $\mu_{j}\left(g_{0}^{\prime}\right)=$ $\exp \left(L_{j}\left(t^{\prime}, \mathfrak{l}\right)\right)$ and $\mu_{j}\left(g_{0}^{\prime \prime}\right)=\exp \left(L_{j}\left(t^{\prime \prime}, s \mathfrak{l}\right)\right)$. A straightforward calculation using these facts, and the definition of $z_{m}$, shows that

$$
q_{j}\left(\mathfrak{l}^{\prime}\right)^{-1}=z_{m}=q_{j}\left(\mathfrak{l}^{\prime \prime}\right)^{-1} \text {. }
$$

But by Lemma $3, \mathfrak{l}_{j}^{\prime}-q_{j}\left(\mathfrak{l}^{\prime}\right)^{-1}=\mathfrak{l}_{j}^{\prime \prime}-q_{j}\left(\mathfrak{l}^{\prime \prime}\right)^{-1}$ since $\mathfrak{l}^{\prime}$ and $\mathfrak{l}^{\prime \prime}$ lie in the same orbit. Thus $\mathfrak{l}_{j}^{\prime}=\mathfrak{l}_{j}^{\prime \prime}$, and $\pi\left(\mathfrak{l}^{\prime}\right)=\pi\left(\mathfrak{l}^{\prime \prime}\right)$.

Note that in the preceding argument we did not assume that $\mathfrak{l}$ and $s \mathfrak{l}$ belonged to the same open set in $F$. Thus the functions $P_{j}$ are independent of the particular open set $O \in F$ chosen, and the proof is complete. Q.E.D.

As above we write $J^{\prime}=\left\{j_{1}<j_{2}<\cdots<j_{h}\right\}$ and recall that $\varphi=\{j \in J$ : $\left.\tau_{j}=3\right\} \subset J^{\prime}$. For each $T \in T(\Omega)$, set $\Omega_{T}=\left\{\mathfrak{l} \in \Omega\right.$ : for each $j_{m} \in \varphi$, $\left.q_{j_{m}}(\mathfrak{l})^{-1} \in T_{m}\right\}$. A cross-section $\Sigma_{T}$ of $\Omega_{T}$ is given by fixing $z \in T$ as follows. For each $j \in J^{\prime}, j=j_{m}$, if $j \notin \varphi$ set $z_{m}=0$, and if $j \in \varphi$, let $z_{m}$ be the (unique) point at which the $m$ th factor of $T$ meets the unit circle. Now by Proposition 2.5 the set $\Sigma_{T}$ of all linear functionals of the form $\sum_{j} P_{j}(z, \mathfrak{l}) f_{j}$, $\mathfrak{l} \in \Omega_{T}$, meets each coadjoint orbit in $\Omega_{T}$ exactly once. Thus the set

$$
\Sigma=\bigcup_{T \in T(\Omega)} \Sigma_{T}
$$

is a cross-section for the orbits in $\Omega$.

For each $j=j_{m} \in \varphi$, let $z_{m}(\mathfrak{l})$ be the point where $q_{j}^{-1}(G \mathfrak{l})$ meets $S^{i_{j}}$. If $j_{m} \notin \varphi$, set $z_{m}(\mathfrak{l})=0$. The mapping $z(\mathfrak{l})=\left(z_{1}(\mathfrak{l}), z_{2}(\mathfrak{l}), \ldots, z_{h}(\mathfrak{l})\right)$ is an analytic function from $\Omega$ into $T(\Omega)$ and $\Sigma$ is the range of the analytic $G$-invariant function $P$ on $\Omega$ defined by $P(\mathfrak{l})=\sum_{j} P_{j}(z(\mathfrak{l}), \mathfrak{l}) f_{j}$.

Let $V=\operatorname{span}_{\mathbf{C}}\left\{f_{j}: \tau_{j} \neq 1\right\}$. It is not at all obvious that $\Sigma$ is an algebraic subset of $\mathfrak{g}^{*}$. The following shows that this is the case. 
Proposition 2.7. For each $j \in l$, there is a complex-valued rational function $p_{j}$, nonsingular on $\Omega$, such that

$\Sigma=\left\{\mathfrak{l} \in V \cap \Omega: p_{j}(\mathfrak{l})=0\right.$, for all $j \in l$, and $\left|q_{j}(\mathfrak{l})\right|^{2}=1$, for all $\left.j \in \varphi\right\}$.

Proof. Fix $j(1 \leq j \leq n)$ such that $j \notin I^{\prime \prime}$; the method of proof is to describe $\pi_{j^{\prime \prime}}(\Sigma)$ in terms of $\pi_{j^{\prime}}(\Sigma)$. From the properties of the functions $P_{j}$ and the definition of $\Sigma$, we have that if $\tau_{j}=0$, then $\pi_{j}(\Sigma)=\left\{\mathfrak{l} \in \pi_{j}(\Omega): \pi_{j^{\prime}}(\mathfrak{l}) \in\right.$ $\left.\pi_{j^{\prime}}(\Sigma)\right\}$, while if $\tau_{j}=1$, then $\pi_{j}(\Sigma)=\pi_{j^{\prime}}(\Sigma)$.

Suppose that $\tau_{j}=2$. There is $k \quad(1 \leq k \leq d / 2)$ such that either $j=i_{k}$ (whence $\left.k \in K_{1}\right)$, or $j=j\left(i_{k}\right)$, where $j^{\prime \prime}=j+1 \notin J$. Let $\mathfrak{l} \in \Omega$, such that $\pi_{j^{\prime}}(\mathfrak{l}) \in \pi_{j^{\prime}}(\Sigma)$. For simplicity of notation set $\rho=\rho_{k-1}(\cdot, \mathfrak{l})$ and let $Y_{1}=\operatorname{Re}\left(\rho\left(Z_{i_{k}}\right)\right), Y_{2}=\operatorname{Im}\left(\rho\left(Z_{i_{k}}\right)\right)$. We show the following.

(i) If $j=i_{k}$, then $\pi_{j^{\prime \prime}}(\mathfrak{l}) \in \pi_{j^{\prime \prime}}(\Sigma)$ if and only if

$$
\mathfrak{l}\left(\left[\rho\left(Z_{j\left(i_{k}\right)}\right), Y_{1}\right]\right) \operatorname{Re}\left(\mathfrak{l}_{j}\right)+\mathfrak{l}\left(\left[\rho\left(Z_{j\left(i_{k}\right)}\right), Y_{2}\right]\right) \operatorname{Im}\left(\mathfrak{l}_{j}\right)=0 .
$$

(ii) If $j=j\left(i_{k}\right)$, then $\pi_{j^{\prime \prime}}(\mathfrak{l}) \in \pi_{j^{\prime \prime}}(\Sigma)$ if and only if

$$
\operatorname{Re}\left(\mathfrak{l}\left(\left[\rho\left(Z_{j\left(i_{k}\right)}\right), Y_{1}\right]\right) \mathfrak{l}_{j}\right)=\operatorname{Re}\left(\mathfrak{l}\left(\left[\rho\left(Z_{j\left(i_{k}\right)}\right), Y_{2}\right]\right) \mathfrak{l}_{j}\right)=0 .
$$

Suppose that $j=i_{k}$. Either $\mathfrak{l}\left(\left[\rho\left(Z_{j\left(i_{k}\right)}\right), Y_{1}\right]\right) \neq 0$ or $\mathfrak{l}\left(\left[\rho\left(Z_{j\left(i_{k}\right)}\right), Y_{2}\right]\right) \neq$ 0 ; assume that the former is true. By (the proof of) Lemma 1.3, we have that the complex numbers $\mathfrak{l}\left(\left[\rho\left(Z_{j\left(i_{k}\right)}, Y_{1}\right]\right)\right.$ and $\mathfrak{l}\left(\left[\rho\left(Z_{j\left(i_{k}\right)}, Y_{2}\right]\right)\right.$ are R-linearly dependent, and we have $Y_{k}(\mathfrak{l}) \in g_{j^{\prime \prime}} \sim g_{j^{\prime}}$ such that $Y_{k}(\mathfrak{l})=\gamma_{1} Y_{1}+\gamma_{2} Y_{2}$ where

$$
\gamma_{1}=\mid \mathfrak{l}\left(\left[\rho\left(Z_{j\left(i_{k}\right)}\right), Y_{1}\right]\right), \quad \gamma_{2}=\mathfrak{l}\left(\left[\rho\left(\bar{Z}_{j\left(i_{k}\right)}\right), Y_{1}\right]\right) \mathfrak{l}\left(\left[\rho\left(Z_{j\left(i_{k}\right)}\right), Y_{2}\right]\right) / \gamma_{1} .
$$

Let $Y_{k}(\mathfrak{l})^{\sim}=\gamma_{2} Y_{1}-\gamma_{1} Y_{2}$; then $\mathfrak{l}\left(\left[\rho\left(Z_{j\left(i_{k}\right)}\right), Y_{k}(\mathfrak{l})^{\sim}\right]\right)=0$, and an easy computation shows

$$
\rho\left(Z_{i_{k}}\right)=\left(\left(\gamma_{1}-i \gamma_{2}\right) Y_{k}(\mathfrak{l})+\left(\gamma_{2}+i \gamma_{1}\right) Y_{k}(\mathfrak{l})^{\sim}\right) /\left|\gamma_{1}+i \gamma_{2}\right| .
$$

Now from the definition of $\Sigma$ we have $\pi_{j^{\prime \prime}}(\mathfrak{l}) \in \pi_{j^{\prime \prime}}(\Sigma)$ iff $\operatorname{Re}\left(c_{j}(\mathfrak{l})^{-1} \mathfrak{l}_{j}\right)=0$ where

$$
\begin{aligned}
c_{j}(\mathfrak{l}) & =\mathfrak{l}\left(\left[Z_{j}, r_{j}(\mathfrak{l})\right]\right)=\mathfrak{l}\left(\left[Z_{i_{k}}, X_{k}(\mathfrak{l})\right]\right) /\left|\mathfrak{l}\left(\left[Z_{i_{k}}, X_{k}(\mathfrak{l})\right]\right)\right| \\
& =\mathfrak{l}\left(\left[\rho\left(Z_{i_{k}}\right), X_{k}(\mathfrak{l})\right]\right) /\left|\mathfrak{l}\left(\left[\rho\left(Z_{i_{k}}\right), X_{k}(\mathfrak{l})\right]\right)\right| .
\end{aligned}
$$

Here $X_{k}(\mathfrak{l})=\operatorname{Re}\left(\mathfrak{l}\left(\left[\rho\left(\bar{Z}_{j\left(i_{k}\right)}\right), Y_{k}(\mathfrak{l})\right]\right) \rho\left(Z_{j\left(i_{k}\right)}\right)\right)$. Since $\mathfrak{l}\left(\left[X_{k}(\mathfrak{l}), Y_{k}(\mathfrak{l})^{\sim}\right]\right)=0$, we conclude that $\pi_{j^{\prime \prime}}(\mathfrak{l}) \in \pi_{j^{\prime \prime}}(\Sigma)$ if and only if $\operatorname{Re}\left(\left(\gamma_{1}-i \gamma_{2}\right) \mathfrak{l}_{j}\right)=0$. But

$$
\begin{aligned}
& \operatorname{Re}\left(\left(\gamma_{1}-i \gamma_{2}\right) \mathfrak{l}_{j}\right)=\mathfrak{l}\left(\left[\rho\left(\bar{Z}_{j\left(i_{k}\right)}\right), \operatorname{Re}\left(\rho\left(Z_{i_{k}}\right)\right)\right]\right) /\left|\mathfrak{l}\left(\left[\rho\left(\bar{Z}_{j\left(i_{k}\right)}\right), \operatorname{Re}\left(\rho\left(Z_{i_{k}}\right)\right)\right]\right)\right| \\
& \quad \times\left(\mathfrak{l}\left(\left[\rho\left(Z_{j\left(i_{k}\right)}\right), \operatorname{Re}\left(\rho\left(Z_{i_{k}}\right)\right)\right]\right) \operatorname{Re}\left(\mathfrak{l}_{j}\right)+\mathfrak{l}\left(\left[\rho\left(Z_{j\left(i_{k}\right)}\right), \operatorname{Im}\left(\rho\left(Z_{i_{k}}\right)\right)\right]\right) \operatorname{Im}\left(\mathfrak{l}_{j}\right)\right) .
\end{aligned}
$$

This proves $(\mathrm{i})$ in the case $\mathfrak{l}\left(\left[\rho\left(Z_{j\left(i_{k}\right)}\right), Y_{1}\right]\right) \neq 0$. Otherwise $\mathfrak{l}\left(\left[\rho\left(Z_{j\left(i_{k}\right)}\right), Y_{2}\right]\right) \neq$ 0 , and a symmetric argument obtains the result.

Suppose that $j=j\left(i_{k}\right)$. As above we have either $\mathfrak{l}\left(\left[\rho\left(Z_{j\left(i_{k}\right)}\right), Y_{1}\right]\right) \neq 0$ or $\mathfrak{l}\left(\left[\rho\left(Z_{j\left(i_{k}\right)}\right), Y_{2}\right]\right) \neq 0$, and we begin by assuming the former. Again we have $\pi_{j^{\prime \prime}}(\mathfrak{l}) \in \pi_{j^{\prime \prime}}(\Sigma)$ if and only if $\operatorname{Re}\left(c_{j}(\mathfrak{l})^{-1} \mathfrak{l}_{j}\right)=0$, where in this case

$$
c_{j}(\mathfrak{l})=\mathfrak{l}\left(\left[Z_{j}, r_{j}(\mathfrak{l})\right]\right)=\mathfrak{l}\left(\left[Z_{j\left(i_{k}\right)}, Y_{k}(\mathfrak{l})\right]\right) /\left|\mathfrak{l}\left(\left[Z_{j\left(i_{k}\right)}, Y_{k}(\mathfrak{l})\right]\right)\right|,
$$

and $Y_{k}(\mathfrak{l})$ is defined as above. Thus $\pi_{j^{\prime \prime}}(\mathfrak{l}) \in \pi_{j^{\prime \prime}}(\Sigma)$ if and only if

$$
\operatorname{Re}\left(\mathfrak{l}\left(\left[\bar{Z}_{j\left(i_{k}\right)}, Y_{k}(\mathfrak{l})\right]\right) \mathfrak{l}_{j}\right)=0 .
$$


But

$$
\begin{aligned}
\mathfrak{l}\left(\left[\bar{Z}_{j\left(i_{k}\right)}, Y_{k}(\mathfrak{l})\right]\right)=\gamma_{1} \mathfrak{l}\left(\left[\bar{Z}_{j\left(i_{k}\right)}, Y_{1}\right]\right)+\gamma_{2} \mathfrak{l}\left(\left[\bar{Z}_{j\left(i_{k}\right)}, Y_{2}\right]\right) \\
=\left|\mathfrak{l}\left(\left[\bar{Z}_{j\left(i_{k}\right)}, Y_{1}\right]\right)\right| \mathfrak{l}\left(\left[\bar{Z}_{j\left(i_{k}\right)}, Y_{1}\right]\right) \\
\quad+\mathfrak{l}\left(\left[\bar{Z}_{j\left(i_{k}\right)}, Y_{1}\right]\right) \mathfrak{l}\left(\left[Z_{j\left(i_{k}\right)}, Y_{2}\right]\right) \mathfrak{l}\left(\left[\bar{Z}_{j\left(i_{k}\right)}, Y_{2}\right]\right) /\left|\mathfrak{l}\left(\left[\bar{Z}_{j\left(i_{k}\right)}, Y_{1}\right]\right)\right| \\
\quad=\left(\left|\mathfrak{l}\left(\left[\bar{Z}_{j\left(i_{k}\right)}, Y_{1}\right]\right)\right|^{2}+\left|\mathfrak{l}\left(\left[\bar{Z}_{j\left(i_{k}\right)}, Y_{1}\right]\right)\right|^{2}\right) \mathfrak{l}\left(\left[\bar{Z}_{j\left(i_{k}\right)}, Y_{1}\right]\right) / \mid \mathfrak{l}\left(\left[Z_{j\left(i_{k}\right)}, Y_{1}\right]\right) .
\end{aligned}
$$

We conclude that $\pi_{j^{\prime \prime}}(\mathfrak{l}) \in \pi_{j^{\prime \prime}}(\Sigma)$ if and only if $\operatorname{Re}\left(\mathfrak{l}\left(\left[\bar{Z}_{j\left(i_{k}\right)}, Y_{1}\right]\right) \mathfrak{l}_{j}\right)=0$. Since $\mathfrak{l}\left(\left[\rho\left(Z_{j\left(i_{k}\right)}\right), Y_{1}\right]\right)$ and $\mathfrak{l}\left(\left[\rho\left(Z_{j\left(i_{k}\right)}\right), Y_{2}\right]\right)$ are $\mathbf{R}$-linearly dependent, we obtain (ii). If we assume that $\mathfrak{l}\left(\left[\rho\left(Z_{j\left(i_{k}\right)}\right), Y_{2}\right]\right) \neq 0$, then a symmetric argument gives the same result.

Finally, suppose that $\tau_{j}=3$. Then $\pi_{j^{\prime \prime}}(\Sigma)=\left\{\mathfrak{l} \in \pi_{j^{\prime \prime}}(\Omega): \pi_{j^{\prime}}(\mathfrak{l}) \in \pi_{j^{\prime}}(\Sigma)\right.$, $\left.\left|q_{j}(\mathfrak{l})\right|^{2}=1\right\}$. This finishes the proof. Q.E.D.

Remark. If $G$ is nilpotent, then for each $j(1 \leq j \leq n) \tau_{j}=0$ or 1 , and the index sets $l$ and $\varphi$ are empty. Thus the usual cross-section $\Sigma=\Omega \cap V$ is obtained.

Finally, we describe the structure of the layer $\Omega$. Let

$$
W(\Omega)=\sum_{j \in J \sim \varphi} \operatorname{Re}_{j}+\sum_{j \in \varphi} \mathbf{R}_{+}^{*} e_{j} .
$$

Let $F$ be the cover of $\Omega_{\alpha} \supset \Omega$ by Zariski open sets obtained in Lemma 1.3, and let $O \in F$. Recall the continuous mapping $P: \Omega \rightarrow \Sigma$. Define a map $\boldsymbol{\Theta}: P^{-1}(\Sigma \cap O) \rightarrow W(\Omega) \times \Sigma \cap O$ as follows. Let $\mathfrak{l} \in P^{-1}(\Sigma \cap O)$, and let $\lambda=P(\mathfrak{l})$. Set $\Theta(\mathfrak{l})=(v, \lambda)$ where $v=\sum_{j \in J \sim \varphi} x_{j} e_{j}+\sum_{j \in \varphi} r_{j} e_{j} \in W(\Omega)$ is defined as follows. Let $j=j_{m} \in J^{\prime}$. Suppose $\tau_{j}=1$. If $\operatorname{dim}\left(L_{j^{\prime}}(\mathfrak{l}) / L_{j^{\prime \prime}}(\mathfrak{l})\right)=1, x_{j}=\mathfrak{l}_{j}$, and if $\operatorname{dim}\left(L_{j^{\prime}}(\mathfrak{l}) / L_{j^{\prime \prime}}(\mathfrak{l})\right)=2$, set $x_{j}+i x_{j+1}=\mathfrak{l}_{j}$. Suppose that $\tau_{j}=2$. Let $z_{j}: O \rightarrow S^{1}$ be the analytic function obtained in Proposition 1.5, and let $s \in G$ such that $s \lambda=\mathfrak{l}$. Then here we set $x_{j}=\operatorname{Re}\left(\nu_{j}(s)^{-1} z_{j}(\lambda)^{-1} \mathfrak{l}_{j}\right)$. It follows from Lemma 2.5 that $x_{j}$ is well defined. If $\tau_{j}=3$, let $r_{j}=\left|q_{j}(\mathfrak{l})\right|^{-1}$. It is evident from Proposition 2.6 that $\Theta$ is an analytic homeomorphism.

The above results are summarized in the following.

Theorem 2.8. Let $G$ be a connected, simply connected exponential solvable Lie group with Lie algebra $\mathfrak{g}$, and let $\left\{X_{1}, X_{2}, \ldots, X_{n}\right\}$ be a good basis of $\mathfrak{g}$, with $\left\{Z_{1}, Z_{2}, \ldots, Z_{n}\right\}$ the corresponding Jordan-Hölder basis of $\mathfrak{g}_{c}$. Let $e_{j}=X_{j}^{*}$ $(1 \leq j \leq n)$ be the dual basis in $\mathfrak{g}^{*}$, and let $f_{j}=Z_{j}^{*}(1 \leq j \leq n)$ be the dual basis in $\mathfrak{g}_{c}^{*}$. Let the subsets $I, I^{\prime}$, and $I^{\prime \prime}$ of $\{1,2, \ldots, n\}$ be defined as above. Then there is a finite partition $\wp$ of $\mathfrak{g}^{*}$, computable in terms of the above Jordan-Hölder basis, with the following properties:

(a) each $\Omega \in \wp$ is $G$-invariant,

(b) for a given $\Omega \in \wp$, the dimension of the coadjoint orbits in $\Omega$ is constant, and $\tau_{j}$ is constant on $\Omega, 1 \leq j \leq n$,

(c) there is an ordering $\Omega_{1}<\Omega_{2}<\cdots<\Omega_{u}$ of $\wp$ such that for each $\Omega$, $\bigcup\left\{\Omega^{\prime}: \Omega^{\prime} \leq \Omega\right\}$ is Zariski open in $\mathfrak{g}^{*}$.

Given $\Omega \in \wp$, let $l=\left\{j \in J: \tau_{j}=2\right\}$ and $\varphi=\left\{j \in J: \tau_{j}=3\right\}$. For each $j \in l$ there is a complex-valued rational function $p_{j}$ and for each $j \in \varphi$, there is a complex-valued rational function $q_{j}$ such that 
(d) $p_{j}$ is nonsingular on $\Omega$, and $q_{j}$ is nonsingular and nonvanishing on $\Omega$ and semi-invariant with multiplier $\mu_{j}^{-1}$,

(e) If $V=\operatorname{span}\left(\left\{f_{j}: \tau_{j} \neq 1\right\}\right)$ then the set

$$
\Sigma=\left\{\mathfrak{l} \in V \cap \Omega: p_{j}(\mathfrak{l})=0, j \in l,\left|q_{j}(\mathfrak{l})\right|^{2}=1, j \in \varphi\right\}
$$

is a cross-section for the coadjoint orbits in $\Omega$, and $\Sigma$ is the range of an analytic $G$-invariant function $P: \Omega \rightarrow \Omega$.

Let $F$ be the cover of $\Omega$ by Zariski open sets as defined in Lemma 1.3, and let $W(\Omega)$ be defined as above. Then

(f) for each $O \in F$, there is an analytic map $\Theta: P^{-1}(O \cap \Sigma) \rightarrow W(\Omega) \times O \cap \Sigma$ such that for each $\lambda \in O \cap \Sigma, \Theta^{-1}(\cdot, \lambda)$ is an analytic diffeomorphisms of $W(\Omega)$ with the coadjoint orbit of $\lambda$. Given $\mathfrak{l} \in P^{-1}(O)$, then $\Theta(\mathfrak{l})=(v, \lambda)$ where $\lambda$ is the unique point in $\Sigma \cap G \mathfrak{l}$, and $v=\sum_{j \in J \sim \varphi} x_{j} e_{j}+\sum_{j \in \varphi} r_{j} e_{j}$ is defined as follows. For each $j \in J$ such that $\tau_{j}=1$, if $j \notin I$, then $x_{j}+i x_{j+1}=\mathfrak{l}_{j}$, while if $j \in I^{\prime}, x_{j}=\mathfrak{l}_{j}$. If $\tau_{j}=2$, then $x_{j}=\operatorname{Re}\left[\nu_{j}(s)^{-1} z_{j}(\lambda)^{-1} \mathfrak{l}_{j}\right]($ where $s \lambda=\mathfrak{l})$, and if $\tau_{j}=3, r_{j}=\left|q_{j}(\mathfrak{l})\right|^{-1}$.

Combining Theorem 2.7 with Corollary 2.8 of [3], we have the following result.

Theorem 2.9. Let $G$ be a connected, simply connected exponential solvable Lie group with Lie algebra $\mathfrak{g}$. Then there is a dense open subset $U$ of $G^{\wedge}$ such that $U$ has the structure of a differentiable manifold.

Proof. Fix a good basis for $\mathfrak{g}$, and the corresponding Jordan-Hölder basis of $\mathfrak{g}_{c}$. Let $\wp$ be the partition of Theorem 2.7, and let $\Omega$ be the minimal element of $\wp$. We show that the cross-section $\Sigma$ for $\Omega$ is a submanifold of $\mathfrak{g}^{*}$.

Recall that $\Sigma$ is the range of the analytic $G$-invariant function $P$ on $\Omega$ defined by $P(\mathfrak{l})=\Sigma_{j} P_{j}(z(\mathfrak{l}), \mathfrak{l}) f_{j}$. We need to show that the rank of $P$ at each $\mathfrak{l}$ is constant $(=n-\operatorname{dim}(G \mathfrak{l}))$. This is verified as follows. Assume that for some $j \notin I^{\prime \prime}, \pi_{j^{\prime}} \circ P$ has constant rank and let $\mathfrak{l} \in \Omega$. We show that if $j \notin J$, then $\operatorname{rank}\left(\pi_{j^{\prime \prime}} \circ P\right)=\operatorname{rank}\left(\pi_{j^{\prime}} \circ P\right)+j^{\prime \prime}-j^{\prime}$, while if $j \in J$, then

(1) if $\tau_{j}=1$, then $\operatorname{rank}\left(\pi_{j^{\prime \prime}} \circ P\right)=\operatorname{rank}\left(\pi_{j^{\prime}} \circ P\right)$,

(2) if $\tau_{j}=2$, then $\operatorname{rank}\left(\pi_{j^{\prime \prime}} \circ P\right)=\operatorname{rank}\left(\pi_{j^{\prime}} \circ P\right)+1$, and

(3) if $\tau_{j}=3$, then $\operatorname{rank}\left(\pi_{j^{\prime \prime}} \circ P\right)=\operatorname{rank}\left(\pi_{j^{\prime}} \circ P\right)+j^{\prime \prime}-j^{\prime}-1$.

Suppose that $j \notin J$. Then by construction, there is an analytic function $Y_{j}(\mathfrak{l})=Y_{j}\left(\mathfrak{l}_{1}, \mathfrak{l}_{2}, \ldots, \mathfrak{l}_{j-1}\right)$ such that $P_{j}(z(\mathfrak{l}), \mathfrak{l})=\mu_{j}(g(z(\mathfrak{l}))) \mathfrak{l}_{j}+Y_{j}(\mathfrak{l})$, where

$$
\begin{aligned}
g(z(\mathfrak{l}))=g\left(\Phi_{1}\left(z_{1}(\mathfrak{l}), \mathfrak{l}\right), \Phi_{2}\left(z_{1}(\mathfrak{l}), z_{2}(\mathfrak{l}), \mathfrak{l}\right), \ldots,\right. \\
\\
\left.\Phi_{k}\left(z_{1}(\mathfrak{l}), z_{2}(\mathfrak{l}), \ldots, z_{m}(\mathfrak{l}), \mathfrak{l}\right), \mathfrak{l}\right),
\end{aligned}
$$

$\left(g(t, \mathfrak{l})\right.$ is as defined in Proposition 2.6). Hence $\operatorname{rank}\left(\pi_{j^{\prime \prime}} \circ P\right)=\operatorname{rank}\left(\pi_{j^{\prime}} \circ P\right)+$ $j^{\prime \prime}-j^{\prime}$.

Suppose that $j \in J$. If $\tau_{j}=1$, then $P_{j}(z(\mathfrak{l}), \mathfrak{l})=0$, from which (1) follows. If $\tau_{j}=2$, then recall that by the proof of Proposition 2.6, for each $\mathfrak{l} \in \Omega$, there is $t \in \mathbf{R}^{k-1} \quad\left(j=j_{k}\right)$ such that we have the formula

$$
P_{j}(z(\mathfrak{l}), \mathfrak{l})=S_{j}(z(\mathfrak{l}), \mathfrak{l})=c_{j}(t, \mathfrak{l}) i \operatorname{Im}\left(c_{j}(t, \mathfrak{l})^{-1}\left[\mathfrak{l}_{j} \exp \left(L_{j}(t, \mathfrak{l})\right)+Y_{j}(t, \mathfrak{l})\right]\right) .
$$

Let $\chi_{j}(z, \mathfrak{l})$ be the $P S^{1}$ valued function of Proposition 2.6. Set

$$
\chi_{j}(\mathfrak{l})=\chi_{j}(z(\mathfrak{l}), \mathfrak{l}) .
$$


Then $\chi_{j}$ is a smooth $G$-invariant function into $P S^{1}$. Now set

$$
A(\mathfrak{l})=A\left(\mathfrak{l}_{1}, \mathfrak{l}_{2}, \ldots, \mathfrak{l}_{j-1}\right)=\left(\pi_{j^{\prime}}(P(\mathfrak{l}))+U\right) \cap \pi_{j^{\prime \prime}}(\Omega),
$$

where $U$ is the (two dimensional) submodule of $W_{j^{\prime \prime}}$ canonically isomorphic to $V_{j^{\prime}} / V_{j^{\prime \prime}}$. Note that $A(\mathfrak{l})=A(s \mathfrak{l}), s \in G$. For each $\mathfrak{l} \in \Omega$, and for any $\mathfrak{l}^{\prime} \in A(\mathfrak{l})$, $r_{j}(\mathfrak{l}) \in L_{j^{\prime}}\left(\mathfrak{l}^{\prime}\right) \sim L_{j^{\prime \prime}}\left(\mathfrak{l}^{\prime}\right)$. It follows immediately that $\zeta_{j}(\mathfrak{l})=\zeta_{j}\left(\mathfrak{l}^{\prime}\right)$. Hence by Lemma 2.5, $\chi_{j}(\mathfrak{l})=\chi_{j}\left(\mathfrak{l}^{\prime}\right)$. Thus the function $\chi_{j}$ depends only on the variables $\mathfrak{l}_{1}, \mathfrak{l}_{2}, \ldots, \mathfrak{l}_{j-1}$. Identify $U$ with the complex numbers in the usual way (so that $x e_{j}+y e_{j+1}$ is identified with $\left.x+i y\right)$. Let $R(\mathfrak{l})=R\left(\mathfrak{l}_{1}, \mathfrak{l}_{2}, \ldots, \mathfrak{l}_{j-1}\right)$ be the R-subspace of $\mathbf{C}$ "through" $\chi_{j}(\mathfrak{l})$. By Proposition 2.6, for each $\mathfrak{l}^{\prime} \in A(\mathfrak{l}), P_{j}\left(\mathfrak{l}^{\prime}\right)$ is on the line $i R(\mathfrak{l})$ and $G^{\prime} \cap A(\mathfrak{l})=\pi_{j^{\prime \prime}}(P(\mathfrak{l}))+R(\mathfrak{l})$. Define $\alpha: \Omega \rightarrow \mathbf{C}$ by $\alpha(\mathfrak{l})=$ the projection of $\mathfrak{l}_{j}$ onto $i R(\mathfrak{l})$ parallel to $R(\mathfrak{l})$. Since $\Omega$ is dense and open in $\mathfrak{g}^{*}, \alpha(A(\mathfrak{l}))$ is a dense subset of $i R(\mathfrak{l})$ and we have

$$
\left(\pi_{j^{\prime \prime}} \circ P\right)(\mathfrak{l})=\left(\pi_{j^{\prime}} \circ P\right)(\mathfrak{l})+\alpha(\mathfrak{l}),
$$

from which assertion (2) follows. If $\tau_{j}=3$, then there is an analytic function $Y_{j}\left(\mathfrak{l}_{1}, \mathfrak{l}_{2}, \ldots, \mathfrak{l}_{j-1}\right)$ such that

$$
P_{j}(z(\mathfrak{l}), \mathfrak{l})=z_{m}(\mathfrak{l})+Y_{j}\left(\mathfrak{l}_{1}, \mathfrak{l}_{2}, \ldots, \mathfrak{l}_{j-1}\right) .
$$

If $j \in I$, then $\Omega=\Omega_{+} \cup \Omega$, where $\Omega_{+}=\left\{\mathfrak{l} \in \Omega: q_{j}(\mathfrak{l})>0\right\}$, and $\Omega_{-}=$ $\left\{\mathfrak{l} \in \Omega: q_{j}(\mathfrak{l})<0\right\}$. The function $z_{m}(\mathfrak{l})$ is constant on each piece, hence $\operatorname{rank}\left(\pi_{j^{\prime \prime}} \circ P\right)=\operatorname{rank}\left(\pi_{j^{\prime}} \circ P\right)$. If $j \notin I$, then by Lemma $2.3, z_{m}$ maps $\Omega$ smoothly onto $S^{1}$. Thus $\operatorname{rank}\left(\pi_{j^{\prime \prime}} \circ P\right)=\operatorname{rank}\left(\pi_{j^{\prime}} \circ P\right)+1$.

By Corollary 2.8 of [3], there is a dense open subset $V_{0}$ of $\mathfrak{g}^{*} / G$ such that $U_{0}=K\left(V_{0}\right)$ is dense and open, and such that $\left.K\right|_{V_{0}}$ is bicontinuous. Set $U=$ $K^{-1}\left(V_{0} \cap(\Omega / G)\right)$. Since $\Omega / G$ is dense and open in $\mathfrak{g}^{*} / G$, it follows that $U$ is a dense, open subset of $G^{\wedge}$ homeomorphic to an open subset of $\Sigma$. Q.E.D.

\section{EXAMPLES}

The following two examples illustrate the cross-sections and bundle structure of the layers. Recall that $\left\{Z_{j}\right\}$ is the Jordan-Hölder basis for $\mathfrak{g}_{c},\left\{f_{j}\right\}$ is the dual basis in $\mathfrak{g}_{c}^{*}$ and $\mathfrak{l}_{j}=\mathfrak{l}\left(Z_{j}\right), \mathfrak{l} \in \mathfrak{g}^{*}$. If $j \in I^{\prime \prime}$ (hence $f_{j}$ is not "real") set $e_{j-1}=2 \operatorname{Re}\left(f_{j}\right), e_{j}=2 \operatorname{Im}\left(f_{j}\right)$. We write the "layers" $\Omega_{\alpha, \varphi}$ as $\Omega_{1}<\Omega_{2}<\cdots$. For each $\Omega_{\alpha, \varphi}$ we exhibit the cover $F=\left\{O_{t}\right\}$ of $\Omega$, and the functions $\phi_{k, t}(\mathfrak{l})$, $X_{k, t}(\mathfrak{l})$, and $Y_{k, t}(\mathfrak{l})$. We then exhibit $W(\Omega), \Sigma$, and the maps $\boldsymbol{\Theta}_{t}$.

Example 1. Let $\mathfrak{g}=\operatorname{span}\{A, X, Y, Z\}$ with nonvanishing brackets $[A, X]=$ $X-Y,[A, Y]=X+Y,[A, Z]=2 Z,[X, Y]=Z$. The Jordan-Hölder basis for $\mathfrak{g}_{c}$ is $Z_{1}=Z, Z_{2}=Y+i X, Z_{3}=Y-i X, Z_{4}=A$.

$\Omega_{1}=\left\{\mathfrak{l}: \mathfrak{l}_{1} \neq 0\right\}: i_{1}=1, i_{2}=2, j\left(i_{1}\right)=4, j\left(i_{2}\right)=3, F=\left\{\Omega_{1}\right\}$ and $Y_{1}(\mathfrak{l})=Z_{1}, X_{1}(\mathfrak{l})=A$,

$Y_{2}(\mathfrak{l})=Y-\left(\left(\operatorname{Re}\left(\mathfrak{l}_{2}\right)+\operatorname{Im}\left(\mathfrak{l}_{2}\right)\right) / 2 \mathfrak{l}_{1}\right) Z, X_{2}(\mathfrak{l})=X-\left(\left(\operatorname{Re}\left(\mathfrak{l}_{2}\right)-\operatorname{Im}\left(\mathfrak{l}_{2}\right)\right) / 2 \mathfrak{l}_{1}\right) Z$.

$W\left(\Omega_{1}\right)=\left\{\mathfrak{l}: \mathfrak{l}_{1}>0\right\}, \Sigma=\left\{f_{1},-f_{1}\right\}, \boldsymbol{\Theta}(\mathfrak{l})=\left(\left|\mathfrak{l}_{1}\right| f_{1}+\mathfrak{l}_{2} f_{2}+\mathfrak{l}_{3} f_{3}+\mathfrak{l}_{4} f_{4}\right.$, $\left.\operatorname{sgn}\left(\mathfrak{l}_{1}\right) f_{1}\right)$.

$\Omega_{2}=\left\{\mathfrak{l}: \mathfrak{l}_{1}=0, \mathfrak{l}_{2} \neq 0\right\}: i_{1}=2, j\left(i_{1}\right)=4$. Again $F=\left\{\Omega_{2}\right\}$, and $Y_{1}(\mathfrak{l})=\left(\operatorname{Re}\left(\mathfrak{l}_{2}\right)+\operatorname{Im}\left(\mathfrak{l}_{2}\right)\right) Y+\left(\operatorname{Re}\left(\mathfrak{l}_{2}\right)-\operatorname{Im}\left(\mathfrak{l}_{2}\right)\right) X, X_{1}(\mathfrak{l})=A$.

$W\left(\Omega_{2}\right)=\left\{r e_{2}+x f_{4}: r>0, x \in R\right\}, \Sigma=\left\{2 \operatorname{Re}\left[\zeta f_{2}\right]: \zeta \in S^{1}\right\}$, and $\boldsymbol{\Theta}(\mathfrak{l})=\left(\left|\mathfrak{l}_{2}\right| e_{2}+\mathfrak{l}_{4} f_{4}, 2 \operatorname{Re}\left[\left(\mathfrak{l}_{2} /\left|\mathfrak{l}_{2}\right|\right) \exp \left(-i \log \left|\mathfrak{l}_{2}\right|\right) f_{2}\right]\right)$. 
$\Omega_{3}=\left\{\mathfrak{l}: \mathfrak{l}_{1}=\mathfrak{l}_{2}=\mathfrak{l}_{3}=0\right\}$ : Here $i(\mathfrak{l})=j(\mathfrak{l})=\varnothing$, and the orbits are single points. We have $F=\left\{\Omega_{3}\right\}, W\left(\Omega_{3}\right)=(0), \Sigma=\Omega_{3}$, and $\boldsymbol{\Theta}(\mathfrak{l})=(0, \mathfrak{l})$.

Example 2. Let $\mathfrak{g}=\operatorname{span}\left\{A, X_{2}, X_{1}, Y_{2}, Y_{1}, T_{4}, T_{3}, T_{2}, T_{1}\right\}$ with nonvanishing brackets $\left[A, X_{1}\right]=X_{1}-X_{2},\left[A, X_{2}\right]=X_{1}+X_{2},\left[A, Y_{1}\right]=Y_{1}-Y_{2}$, $\left[A, Y_{2}\right]=Y_{1}+Y_{2},\left[A, T_{3}\right]=2 T_{3}-2 T_{4},\left[A, T_{4}\right]=2 T_{3}+2 T_{4},\left[A, T_{1}\right]=$ $2 T_{1},\left[A, T_{2}\right]=2 T_{2},\left[X_{1}, Y_{1}\right]=(1 / 2)\left(T_{1}+T_{3}\right),\left[X_{1}, Y_{2}\right]=(1 / 2)\left(T_{4}-T_{2}\right)$, $\left[X_{2}, Y_{1}\right]=(1 / 2)\left(T_{4}+T_{2}\right),\left[X_{2}, Y_{2}\right]=(1 / 2)\left(T_{1}-T_{3}\right)$. The Jordan-Hölder basis here is $Z_{1}=T_{1}, Z_{2}=T_{2}, Z_{3}=T_{3}+i T_{4}, Z_{4}=T_{3}-i T_{4}, Z_{5}=Y_{1}+i Y_{2}$, $Z_{6}=Y_{1}-i Y_{2}, Z_{7}=X_{1}+i X_{2}, Z_{8}=X_{1}-i X_{2}, Z_{9}=A$. For each $\Omega_{t}$ one has $F=\left\{\Omega_{t}\right\}$, except for $\Omega_{3}$; in this example we focus only on $\Omega_{3}$. We begin by listing the first three layers.

$\Omega_{1}=\left\{\mathfrak{l}: \mathfrak{l}_{1} \neq 0,\left|\mathfrak{l}_{1}+i_{2}\right| \neq\left|\mathfrak{l}_{3}\right|\right\}: i_{1}=1, j\left(i_{1}\right)=9, i_{2}=5, j\left(i_{2}\right)=7$, $i_{3}=6, j\left(i_{3}\right)=8$,

$\Omega_{2}=\left\{\mathfrak{l}: \mathfrak{l}_{1}=0, \mathfrak{l}_{2} \neq 0,\left|\mathfrak{l}_{1}+i \mathfrak{l}_{2}\right| \neq\left|\mathfrak{l}_{3}\right|\right\}: i_{1}=2, j\left(i_{1}\right)=9, i_{2}=5$, $j\left(i_{2}\right)=7, i_{3}=6, j\left(i_{3}\right)=8$,

$\Omega_{3}=\left\{\mathfrak{l}: \mathfrak{l}_{1} \neq 0,\left|\mathfrak{l}_{1}+i \mathfrak{l}_{2}\right|=\left|\mathfrak{l}_{3}\right|\right\}: i_{1}=1, j\left(i_{1}\right)=9, i_{2}=5, j\left(i_{2}\right)=7$.

Set $\beta_{t}(\mathfrak{l})=\mathfrak{l}\left(\left[X_{1}+i X_{2}, Y_{t}\right]\right), t=1,2$. Applying the proof of Lemma 1.3 to $\Omega=\Omega_{3}$, we have $F=\left\{O_{1}, O_{2}\right\}$ where $O_{t}=\left\{\mathfrak{l} \in \Omega: \beta_{t}(\mathfrak{l}) \neq 0\right\}$. For $t=1,2$, we have

$$
\begin{aligned}
& \phi_{1, t}(\mathfrak{l})=1, Y_{1, t}(\mathfrak{l})=Z_{1}, X_{1, t}(\mathfrak{l})=A, \\
& \phi_{2, t}(\mathfrak{l})=\beta_{t}(\mathfrak{l}) /\left|\beta_{t}(\mathfrak{l})\right|, Y_{2, t}(\mathfrak{l})=\phi_{2, t}(\mathfrak{l})^{-1}\left(\beta_{1}(\mathfrak{l}) Y_{1}+\beta_{2}(\mathfrak{l}) Y_{2}\right), \\
& X_{2, t}(\mathfrak{l})=\operatorname{Re}\left[\mathfrak{l}\left(\left[X_{1}-i X_{2}, Y_{2, t}(\mathfrak{l})\right]\right) \rho_{1}\left(X_{1}+i X_{2}, \mathfrak{l}\right)\right] \\
& W=W(\Omega)=\left\{r_{1} e_{1}+x_{1} e_{5}+x_{2} e_{7}+x_{3} e_{9}: r_{1}>0, x_{i} \in \mathbf{R}\right\}, \text { and } \\
& \Sigma=\left\{\mathfrak{l} \in \Omega \cap V: \mathfrak{l}_{1}= \pm 1, \beta_{1}(l) \operatorname{Re}\left(\mathfrak{l}_{5}\right)+\beta_{2}(\mathfrak{l}) \operatorname{Im}\left(\mathfrak{l}_{5}\right)=0, \operatorname{Re}\left(\beta_{1}(\mathfrak{l}) \mathfrak{l}_{7}\right)=\right.
\end{aligned}
$$
$\left.\operatorname{Re}\left(\beta_{2}(\mathfrak{l}) \mathfrak{l}_{7}\right)=0\right\}$ where $V=\operatorname{span}\left\{f_{j}: 1 \leq j \leq 8\right\}$.

Set $z_{5}(\mathfrak{l})=\phi_{2, t}(\mathfrak{l})^{-1} \mathfrak{l}_{3} /\left|\mathfrak{l}_{3}\right|, \quad z_{7}(\mathfrak{l})=z_{5}(\mathfrak{l})\left(\mathfrak{l}_{1}+i \mathfrak{l}_{2}\right) /\left|\mathfrak{l}_{1}+i \mathfrak{l}_{2}\right|$. Then $\boldsymbol{\theta}_{t}(\mathfrak{l})=$ $\left(\left(\left|\mathfrak{l}_{1}\right| e_{1}+\operatorname{Re}\left(z_{5}(\mathfrak{l})^{-1} \mathfrak{l}_{5}\right) e_{5}+\operatorname{Re}\left(z_{7}(\mathfrak{l})^{-1} \mathfrak{l}_{7}\right) e_{7}+\mathfrak{l}_{9} e_{9}\right), \lambda\right)$ where

$$
\begin{aligned}
\lambda= & P(\mathfrak{l})=\operatorname{sgn}\left(\mathfrak{l}_{1}\right) f_{1}+\left|\mathfrak{l}_{1}\right|^{-1} \mathfrak{l}_{2} f_{2}+2 \operatorname{Re}\left[\left|\mathfrak{l}_{1}\right|^{-1-i} \mathfrak{l}_{3} f_{3}\right] \\
& +2 \operatorname{Re}\left[z_{5}(\mathfrak{l}) \operatorname{Im}\left(z_{5}(\mathfrak{l})^{-1} \mathfrak{l}_{5}\right) f_{5}\right]+2 \operatorname{Re}\left[z_{7}(\mathfrak{l}) \operatorname{Im}\left(z_{7}(\mathfrak{l})^{-1} \mathfrak{l}_{7}\right) f_{7}\right] .
\end{aligned}
$$

Note that for $\mathfrak{l} \in \Omega, \operatorname{Im}\left(\mathfrak{l}_{3} /\left(\mathfrak{l}_{1}+i \mathfrak{l}_{2}\right)\right) \neq 0$. It is easily seen that for each $\lambda \in \Sigma$ and $r_{1} e_{1}+x_{1} e_{5}+x_{2} e_{7}+x_{3} e_{9} \in W$, if $\operatorname{Im}\left(l_{3} /\left(\mathfrak{l}_{1}+i l_{2}\right)\right)>0$ then

$$
\Theta_{1}\left(\Theta_{2}^{-1}\left(r_{1} e_{1}+x_{1} e_{5}+x_{2} e_{7}+x_{3} e_{9}, \lambda\right)\right)=\left(r_{1} e_{1}+x_{1} e_{5}+x_{2} e_{7}+x_{3} e_{9}, \lambda\right)
$$

while if $\operatorname{Im}\left(\mathfrak{l}_{3} /\left(\mathfrak{l}_{1}+i \mathfrak{l}_{2}\right)\right)<0$ then

$$
\Theta_{1}\left(\Theta_{2}^{-1}\left(r_{1} e_{1}+x_{1} e_{5}+x_{2} e_{7}+x_{3} e_{9}, \lambda\right)\right)=\left(r_{1} e_{1}-x_{1} e_{5}-x_{2} e_{7}+x_{3} e_{9}, \lambda\right) \text {. }
$$

\section{CONCLUDING REMARKS}

The layering $\wp$ and a computable cross-section for each layer in $\wp$ can be obtained for other classes of Lie groups. However, there seems to be no version of Proposition 2.6 for any general class of nonexponential groups. (This is due in part to the fact that stabilizers are not connected, and hence there may be $\mathfrak{l} \in \mathfrak{g}^{*}$ such that $\pi_{j}(\mathfrak{l})+U \cap \pi_{j}(G \mathfrak{l})$ is not simply connected.) Therefore the exponential case appears to be the most general case for which an analogue of Proposition 1.1, Chapter II of [18] holds. For general solvable groups the plan is simple to state: determine layers where the orbit structure in the affine sets 
$\pi_{j}(\mathfrak{l})+U$ is consistent, then compute a cross-section for the coadjoint orbits inductively. The main problem with this is that in general it is difficult to give a reasonable (or useful) description of the layers and their cross-sections. We believe such a description can be obtained for the class of algebraic solvable Lie groups. We shall address this case in a subsequent paper (with R. Penney).

In another direction, the extension of these results to arbitrary exponential solvable Lie group actions appears quite possible. The main problem here is extending Lemma 1.3; some technical details remain to be worked out.

We thank the referee for several important and helpful suggestions.

\section{REFERENCES}

1. I. Brown, Dual topology of a nilpotent Lie group, Ann. Sci. École Norm. Sup. 6 (1973), 407-411.

2. L. Corwin, F. P. Greenleaf, and G. Grelaud, Direct integral decompositions and multiplicities for induced representations of nilpotent Lie groups, Trans. Amer. Math. Soc. 304 (1988), 549-583.

3. B. Currey, On the dual of an exponential solvable Lie group, Trans. Amer. Math. Soc. 309 (1988), 295-307.

4. B. Currey and R. Penney, The structure of the space of coadjoint orbits of a completely solvable Lie group, Michigan Math. J. 36 (1989), 309-320.

5. M. Duflo and M. Rais, Sur Tanalyse harmonique sur les groupes de Lie resolubles, Ann. Sci. École Norm. Sup. 9 (1976), 107-144.

6. H. Fujiwara, Representations monomiales des groupes de Lie resolubles exponentiels, Progress in Math. 82 (1990), 61-84.

7. __ Sur le dual d'un group de Lie resoluble exponentiel, J. Math. Soc. Japan 36 (1984), 629-636.

8. __ Sur les restrictions des representations unitaires des groupes de Lie resolubles exponentiels (to appear).

9. R. Lipsman, Induced representations of completely solvable Lie groups, Ann. Scuola Norm. Sup. Pisa Sci. Fis. Mat. (4) 17 (1990), 127-164.

10. 313 (1989), 433-473.

11. _ Restricting representations of completely solvable Lie groups, Canad. J. Math. 42 (1990), 790-824.

12. N. V. Pedersen, Geometric quantization and the universal enveloping algebra of nilpotent Lie groups, Trans. Amer. Math. Soc. 315 (1989), 511-563.

13. __ On the characters of exponential solvable Lie groups, Ann. Sci. École Norm. Sup. 17 (1984), 1-29.

14. __, On the infinitessimal kernel of irreducible representations of nilpotent Lie groups, Bull. Soc. Math. France 112 (1984), 423-467.

15. __ Semicharacters and solvable Lie groups, Math. Ann. 247 (1980), 191-244.

16. L. Pukanszky, On the characters and the Plancherel formula of nilpotent Lie groups, J. Funct. Anal. 1 (1967), 255-280.

17. __ On the unitary representations of exponential groups, J. Funct. Anal. 2 (1968), 73-113.

18. _, Unitary representations of solvable Lie groups, Ann. Sci. École Norm. Sup. 4 (1971), 457-608.

Department of Mathematics, St. Louis University, St. Louis, Missouri 63103

E-mail address: curreybn@sluvca.slu.edu 